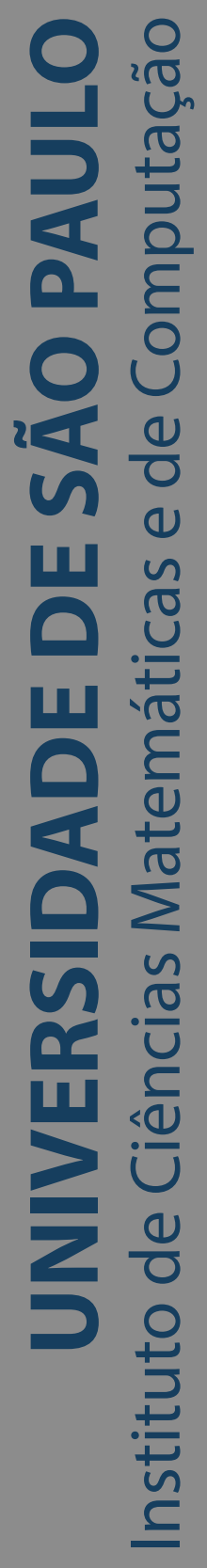

\title{
Introduction to Morse theory and Morse homology
}

\section{Julian David Espinel Leal}

Dissertação de Mestrado do Programa de Pós-Graduação em Matemática (PPG-Mat) 

SERVIÇO DE PÓS-GRADUAÇÃO DO ICMC-USP

Data de Depósito:

Assinatura:

Julian David Espinel Leal

Introduction to Morse theory and Morse homology

Dissertation submitted to the Instituto de Ciências Matemáticas e de Computação - ICMC-USP - in accordance with the requirements of the Mathematics Graduate Program, for the degree of Master in Science. FINAL VERSION

Concentration Area: Mathematics

Advisor: Prof. Dr. Raimundo Nonato Araújo dos Santos 
Ficha catalográfica elaborada pela Biblioteca Prof. Achille Bassi e Seção Técnica de Informática, ICMC/USP, com os dados inseridos pelo(a) autor(a)

Espinel Leal, Julian David Introduction to Morse theory and Morse homology / Julian David Espinel Leal; orientador Raimundo Nonato Araújo dos Santos. -- São Carlos, 2021. $72 \mathrm{p}$.

Dissertação (Mestrado - Programa de Pós-Graduação em Matemática) -- Instituto de Ciências Matemáticas e de Computação, Universidade de São Paulo, 2021.

1. Teoria de Morse. 2. Homologia de Morse. 3. Funções de Morse-Smale. 4. Topologia da variedade. I. Nonato Araújo dos Santos, Raimundo, orient. II. Título.

Bibliotecários responsáveis pela estrutura de catalogação da publicação de acordo com a AACR2: Gláucia Maria Saia Cristianini - CRB - 8/4938 Juliana de Souza Moraes - CRB - 8/6176 


\title{
Julian David Espinel Leal
}

\section{Introdução à teoria de Morse e homologia de Morse}

\author{
Dissertação apresentada ao Instituto de Ciências \\ Matemáticas e de Computação - ICMC-USP, \\ como parte dos requisitos para obtenção do título \\ de Mestre em Ciências - Matemática. VERSÃO \\ REVISADA \\ Área de Concentração: Matemática \\ Orientador: Prof. Dr. Raimundo Nonato Araújo \\ dos Santos
}





\section{RESUMO}

JULIAN E. Introdução à teoria de Morse e homologia de Morse. 2021. 74 p. Dissertação (Mestrado em Ciências - Matemática) - Instituto de Ciências Matemáticas e de Computação, Universidade de São Paulo, São Carlos - SP, 2021.

Neste trabalho apresentamos um estudo da Teoria de Morse com o objetivo de introduzir o teorema da homologia de Morse como sua extensão natural. Para isso provamos o clássico teorema de Morse que garante que uma função de Morse numa variedade determina sua topologia como CW-complexo por meio de seus pontos críticos. Na sequência introduzimos os polinômios de Morse e Poincaré, suas relações, e as funcões de Morse perfeitas que mostram quando o número de pontos críticos não degenerados de índice $k$ é exatamente igual o $k$-ésimo número de Betti da variedade. Por fim, apresentamos as variedades estáveis e instáveis determinadas pelo fluxo gradiente de uma função de Morse-Smale e o complexo de cadeia de Morse-Smale-Witten cuja homologia é isomorfa à homologia singular da variedade.

Palavras-chave: Teoria de Morse, Homologia de Morse, Funções de Morse-Smale, Topologia da variedade. 



\section{ABSTRACT}

JULIAN E. Introduction to Morse theory and Morse homology. 2021. 74 p. Dissertação (Mestrado em Ciências - Matemática) - Instituto de Ciências Matemáticas e de Computação, Universidade de São Paulo, São Carlos - SP, 2021.

In this work we present a study of Morse theory with the aim of introducing the Morse homology theorem as its natural extension. For this we prove the classic Morse theorem, which states that a Morse function defined on a manifold determines its topology as CW-complex through its critical points. Next, we introduce the Morse and Poincaré polynomials, their relations, and the perfect Morse functions that show when the number of non-degenerate critical points is equal to the k-th Betti number of the manifold. Finally, we present the stable and unstable manifolds given by the gradient flow of a Morse-Smale function and the Morse-Smale-Witten chain complex whose homology is isomorphic to the singular homology of the manifold.

Keywords: Morse theory, Morse homology, Morse-Smale functions, Topology of manifold. 



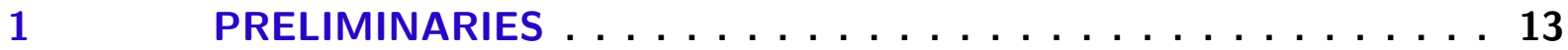

$1.1 \quad$ Basic definitions and facts $\ldots \ldots \ldots \ldots \ldots \ldots$

1.1.1 Derivations . . . . . . . . . . . . . . . . . . . . . 14

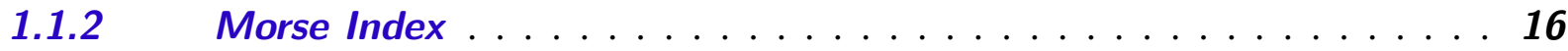

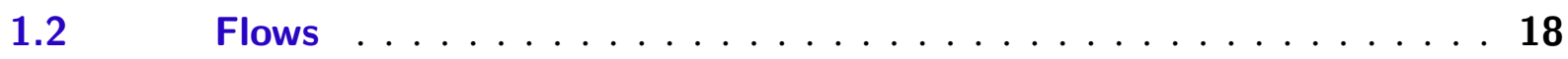

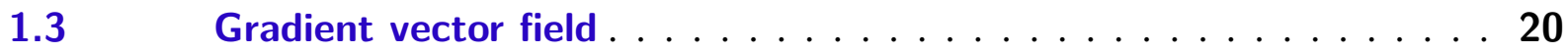

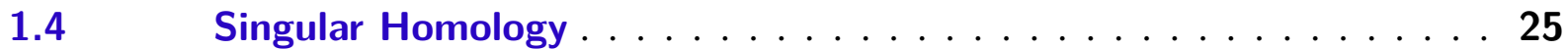

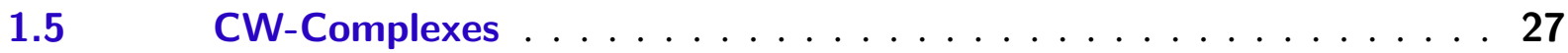

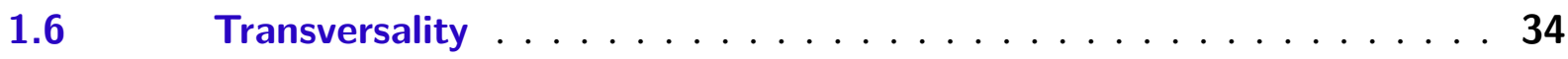

$1.7 \quad$ Intersection number . . . . . . . . . . . . . . . . . 35

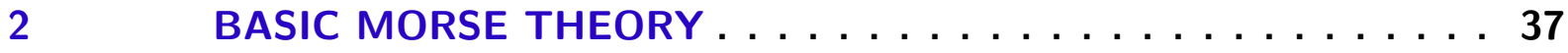

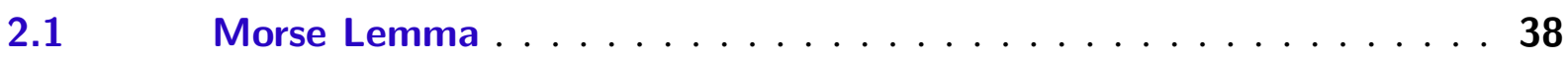

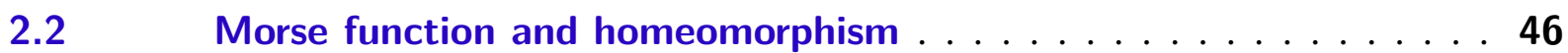

$2.3 \quad$ Morse functions and homotopy type $\ldots \ldots \ldots \ldots \ldots \ldots$

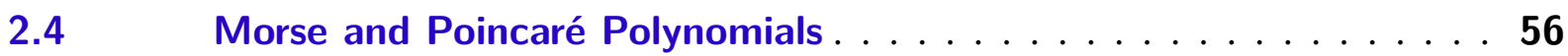

2.4.1 Perfect Morse functions and examples . . . . . . . . . . .60

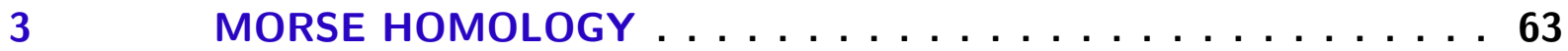

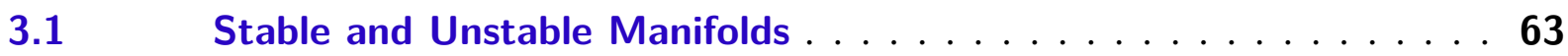

3.1.1 Morse-Smale functions . . . . . . . . . . . . . . . . . . 66

3.2 Morse Homology Theorem $\ldots \ldots \ldots \ldots \ldots \ldots$

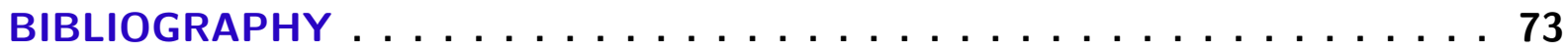



The first approaches to Morse Theory was done by Marston Morse (MORSE, 1929). The idea was to study the topology of a smooth manifold $M$ using a Morse function on it and the information at each critical point of the function. This was done considering the topological spaces $M^{a}=f^{-1}((-\infty, a])$ where $f: M \rightarrow \mathbb{R}$ is a Morse function and noticing that if there is no critical values between $a$ and $b$ with $a<b$ then the manifolds $M^{a}$ and $M^{b}$ are diffeomorphic. Moreover, the space $M^{a}$ is a deformation retract of $M^{b}$. From this, the topology of $M^{t}$ can only change when $t$ passes through a critical value of $f$. If we give the condition that $f$ is a Morse function the first important result in Morse theory comes up. It states that if $c$ is a critical value of $f$ and $\varepsilon$ is small enough, then the space $M^{c+\varepsilon}$ has the same homotopy type of $M^{c-\varepsilon}$ attaching a $k$-cell for each critical point of index $k$ in $f^{-1}(c)$. That leads us to the main Theorem of Chapter 2 of this work which states that $M$ has the same homotopy type of a CW-complex $X$ with an $\lambda$-cell for each critical point of $f$ of index $\lambda$. These results give rise to the well-known Morse inequalities which relate the Betti numbers $b_{i}$ of a $m$-manifold $M$ and the number of critical points $v_{i}$ of index $i$ of a Morse function on it:

$$
v_{k}-v_{k-1}+\cdots+(-1)^{k} v_{0} \geq b_{k}-b_{k-1}+\cdots+(-1)^{k} b_{0}, \text { for } k=0, \ldots, m .
$$

A natural question that arises from these inequalities is whether or not the equality in (1) holds? When $k=m$ it is known that equality holds and $\chi(M)=\sum_{i=0}^{m}(-1)^{i} v_{i}$.

On the other hand, the CW-complex $X=X^{m} \supset X^{m-1} \supset \cdots \supset X^{0}$ provides the following chain complex

$$
0 \rightarrow H_{m}\left(X^{m}, X^{m-1}\right) \rightarrow H_{m-1}\left(X^{m-1}, X^{m-2}\right) \rightarrow \cdots \rightarrow H_{1}\left(X^{1}, X^{0}\right) \rightarrow H_{0}\left(X^{0}\right) \rightarrow 0
$$

and the homology of this chain complex is isomorphic to the singular homology of $M$. To calculate its homology we need to know well the boundary operator, which in turn we need to study the attaching maps given by the cell structure of $X$.

The first studies of that operator was made by Thom (THOM, 1949), Milnor (MILNOR, 2016) and Smale (SMALE, 1967). From these studies originated the Morse homology theory.

The aim of this work is to provide the basic tools and techniques in order to introduce the Morse Homology Theorem. For this we organize this work as described below.

In Chapter 1, we give all the definitions and basic theorems of topology and geometry that we will be useful in the development of this work, such as derivations, Morse index, flows (in particular, the gradient vector field), singular homology (in particular for CW-complexes), transversality and intersection number. 
In Chapter 2, we start reviewing the classical Morse theory and presenting examples and applications. Also we prove the classical Morse theorem that states that a smooth manifold has the same homotopy type of a CW-complex where its cells is in correspondence with the number of critical points of a Morse function on it. As consequence we obtain the Reeb's Theorem, the Morse inequalities and its relations with the Poincare and Morse polynomials and finishing with a brief study of perfect Morse function.

In Chapter 3 we present properties of the gradient vector field of a Morse-Smale function defined on a manifold. We introduce the stable and unstable manifolds in order to show that there are finitely many flow lines between two critical points of consecutive Morse index. We finish this chapter by defining the Morse-Smale-Witten chain complex in order to introduce the Morse Homology theorem which says that the homology of the Morse-Smale-Witten chain complex is isomorphic to the singular homology of the manifold. 
CHAPTER

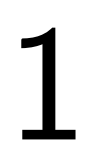

PRELIMINARIES

In this chapter we will give some definitions, notations and facts of geometry and topology that we will be useful for the development of this work. We present some classical facts and for some of them we will include their proofs. In this chapter the definitions and basic results comes from the following references (LEE, 2013), (GREENBERG, 1967), (HATCHER, 2002), (MILNOR, 2016), (BREDON, 1993), (GUILLEMIN; POLLACK, 1974).

\subsection{Basic definitions and facts}

Let $M$ be smooth manifold of dimension $m$ and let $p \in M$. Let $V_{p} M$ be the set of smooth curves $\gamma: J \rightarrow M$ where $J$ is an interval containing 0 and $\gamma(0)=p$. Let $(U, \phi)$ be a chart in $M$ such that $\phi(p)=0$ and $\phi(U)=\mathbb{R}^{m}$. Define an equivalence relation $\sim$ in $V_{p} M$ as follows. Given $\gamma, \alpha \in V_{p} M$, let us say that $\gamma \sim \alpha$ if $(\phi \circ \gamma)^{\prime}(0)=(\phi \circ \alpha)^{\prime}(0)$. With this let us define $T_{p} M:=V_{p} M / \sim$, i.e., $T_{p} M$ is the set of equivalence classes in $V_{p} M$. It is a known fact that $T_{p} M$ can be endowed with a real vector space structure of dimension $m$. The vector space $T_{p} M$ is called tangent space of $M$ at $p$.

Let $N$ be a smooth manifold. Then, a smooth map $g: M \rightarrow N$ induces a linear map $d_{p} g: T_{p} M \rightarrow T_{g(p)} N,[\gamma] \mapsto[g \circ \gamma]$, called the differential of $g$ at $p$. When there is not ambiguity in the choice of the point $p$ we will denote $d_{p} g$ by $g_{*}$.

The set $T M:=\bigsqcup_{p \in M} T_{p} M$ is called the tangent bundle of $M$. It can be shown that $T M$ is a smooth manifold of dimension $2 m$. A point of $T M$ is denoted by $(p, v)$ where $p \in M$ and $v \in T_{p} M$, when there is not confusion we denote $(p, v)$ by $v_{p}$. Hence, there is a smooth projection map $\pi: T M \rightarrow M,(p, v) \mapsto p$. A smooth map $X: M \rightarrow T M$ such that $\pi \circ X=i d_{M}$ is called tangent vector field on $M$ or a smooth section of $T M$. Let us denote the image $X(p)$ by $X_{p}$ that is a tangent vector of $M$ at $p$.

Let $f: M \rightarrow \mathbb{R}$ be a smooth function and let $\left(U, \phi=\left(x^{1}, \ldots, x^{m}\right)\right)$ be a chart that we 
also call a local coordinate system. In order to simplify notation denote $f \circ \phi^{-1}\left(x^{1}, \ldots, x^{m}\right)$ by $f\left(x^{1}, \ldots, x^{m}\right)$ and denote the partial derivation $\frac{\partial\left(f \circ \phi^{-1}\right)}{\partial x^{i}}(\phi(p))$ by $\frac{\partial f}{\partial x^{i}}(p)$ for $i=1, \ldots, m$.

Throughout this section we fix the local coordinate system $\left(U, \phi=\left(x^{1}, \ldots, x^{m}\right)\right)$ when necessary.

Definition 1.1. We say that a point $p \in M$ is a regular point of $g$ if the induced linear map $d_{p} g$ has maximal rank. Otherwise we said that $p$ is a critical point of $g$. A point $c \in N$ is called regular value of $g$ if $g^{-1}(c)=\varnothing$ or every point in $g^{-1}(c)$ is a regular point. Otherwise $c$ is called a critical value of $g$.

Let $f: M \rightarrow \mathbb{R}$ be a smooth function. Let us remark that a point $p \in M$ is a critical point of $f$ if and only if the induced linear map $d_{p} f$ is the zero map i.e., $\frac{\partial f}{\partial x^{i}}(p)=0$ for $i=1, \ldots, n$. It is easy to see that this equivalence does not depend of the choice of the coordinate system. Given $a \in \mathbb{R}$ let us define $M^{a}:=f^{-1}((-\infty, a])$. With this notation we state the following proposition that is a consequence of the Implicit Function Theorem and we present its proof by completeness.

Proposition 1.2. Let $f: M \rightarrow \mathbb{R}$ be a smooth function and let $a \in \mathbb{R}$ be a regular value of $f$. Then $M^{a}$ is a smooth $m$-manifold with boundary $f^{-1}(a)$. Moreover $f^{-1}(a)$ is a $m$-1-manifold.

Proof. For the first part it is enough to give an atlas for $M^{a}$. Since int $M^{a}=f^{-1}((-\infty, a))$ is an open set of $M$, we have that it is a smooth manifold. Now we will give a boundary charts for the points on $f^{-1}(a)$. For each point $p \in f^{-1}(a)$ we have a chart $\left(V, \varphi=\left(x^{1}, \ldots, x^{m}\right)\right)$ of the manifold $M$. Since $a$ is a regular value, we conclude from the Implicit Function Theorem that there exists an open neighborhood $V_{1} \times V_{2} \subset \mathbb{R}^{m-1} \times \mathbb{R}$ of $\varphi(p)$ and a smooth function $g: V_{1} \rightarrow V_{2}$ such that $f \circ \varphi^{-1}(x, y)=a$ if and only if $y=g(x)$. Define the local coordinate system $\varphi^{\prime}=\left(x^{1}, \ldots, x^{m-1}, x^{m}-g\left(x^{1}, \ldots, x^{m-1}\right)\right)$ in a open neighborhood $V^{\prime} \subset V$ of $p$ such that $\varphi\left(V^{\prime}\right) \subset$ $V_{1} \times V_{2}$. Then for all point $q \in V^{\prime} \cap f^{-1}(a)$ we have that $\varphi(q)=\left(x^{1}, \ldots, x^{m-1}, 0\right)$. Therefore $\left(V^{\prime} \cap M^{a},\left.\varphi^{\prime}\right|_{V^{\prime} \cap M^{a}}\right)$ is a boundary chart of $M^{a}$ at $p$. From this we conclude that $M^{a}$ is a smooth manifold with boundary $\partial M^{a}=\left\{p \in M^{a} \mid f(p)=a\right\}$. For the second part we have from the Level Set Theorem that $\partial M^{a}=f^{-1}(a)$ is a manifold of dimension $m-1$.

\subsubsection{Derivations}

Let us denote the vector space of the smooth functions $f: M \rightarrow \mathbb{R}$ by $C^{\infty}(M)$. A map $v: C^{\infty}(M) \rightarrow \mathbb{R}$ is called a derivation of $M$ at $p$ if it is a linear map over $\mathbb{R}$ and satisfies the following identity

$$
v(f g)=f(p) v(g)+v(f) g(p) \text { where } f, g \in C^{\infty}(M)
$$

which is called the Leibniz Rule.

Denote the set of derivation of $f$ at $p$ by $D_{p} M$ which endowed with the operations

$$
(v+w)(f)=v(f)+v(f),(r w)(f)=r w(f) \text { where } v, w \in D_{p} M \text { and } r \in \mathbb{R}
$$


is a vector space of dimension $m$.

Example 1.3. Let $M=\mathbb{R}^{m}$ and let $p \in \mathbb{R}^{m}$. The operator

$$
\begin{aligned}
\left.\frac{\partial}{\partial x_{i}}\right|_{p}: C^{\infty}\left(\mathbb{R}^{m}\right) & \longrightarrow \mathbb{R} \\
f & \longmapsto \frac{\partial f}{\partial x_{i}}(p)
\end{aligned}
$$

is a derivation of $\mathbb{R}^{m}$ at $p$.

Proposition 1.4. The spaces $T_{p} M$ and $D_{p} M$ are isomorphic.

The isomorphism between $T_{p} M$ and $D_{p} M$ is given by the linear map $L: T_{p} M \rightarrow D_{p} M$, $[\gamma] \mapsto L[\gamma]$, where $L[\gamma]: C^{\infty}(M) \rightarrow \mathbb{R}, f \mapsto(f \circ \gamma)^{\prime}(0)$. Hence, another equivalent definition of tangent space is the vector space of derivations which we will use more in this work.

A map $X: C^{\infty}(M) \rightarrow C^{\infty}(M)$ is called a derivation on $M$ if it is a linear map over $\mathbb{R}$ and satisfies the Leibniz rule:

$$
X(f g)=f X(g)+X(f) g \text { for all } f, g \in C^{\infty}(M) .
$$

In a similar way as it was done with the tangent space, we can identify the set of tangent vector fields on $M$ with the set of derivations on $M$, as follows. If we denote by $\mathfrak{X}(M)$ the set of smooth vector fields on $M$ and by $\mathfrak{D}(M)$ the set of derivations on $M$, then we have a bijection $\Phi: \mathfrak{X}(M) \rightarrow \mathfrak{D}(M)$ where for each $V \in \mathfrak{X}(M), \Phi(V): C^{\infty}(M) \rightarrow C^{\infty}(M)$ is a derivation defined by $\Phi(V)(f)(p)=V_{p}(f)$. On the other hand, for each $X \in \mathfrak{D}(M), \Phi^{-1}(X)$ is the unique smooth vector field such that $\left(\Phi^{-1}(X)\right)_{p}(f)=X(f)(p)$. See (LEE, 2013, Proposition 8.15). Thus, in this work we treat vector fields on $M$ and derivation on $M$ indistinctly.

Definition 1.5. A critical point $p$ of a smooth function $f: M \rightarrow \mathbb{R}$ is called non-degenerate if the matrix $\left(\frac{\partial f}{\partial x^{i} \partial x^{j}}(p)\right)$ is non-singular for any local coordinate system $\left(x^{1}, \ldots, x^{m}\right)$ in a neighborhood $U$ of $p$.

The above definition does not depend on the choice of the local coordinate system as the following proposition shows.

Proposition 1.6. Let $p$ be a critical point of a smooth function $f$ on a $m$-manifold $M$. If $\varphi=\left(x^{1}, \ldots, x_{m}\right)$ and $\psi=\left(y^{1}, \ldots, y^{m}\right)$ are two local coordinate system in a neighborhood of $p$, then $\left(\frac{\partial^{2} f}{\partial x^{i} \partial x^{j}}(p)\right)$ is non-singular if and only if $\left(\frac{\partial^{2} f}{\partial y^{i} \partial y^{j}}(p)\right)$ is non-singular.

Proof. Denote $f_{\varphi}=f \circ \varphi^{-1}, f_{\psi}=f \circ \psi^{-1}$ and $\Phi=\psi \circ \varphi^{-1}$. Then we have that $f_{\varphi}=f_{\psi} \circ \Phi$

$$
\begin{aligned}
\frac{\partial^{2} f_{\varphi}}{\partial x^{i} \partial x^{j}} & =\frac{\partial}{\partial x^{j}}\left(\frac{\partial\left(f_{\psi} \circ \Phi\right)}{\partial x^{i}}\right)=\frac{\partial}{\partial x^{j}}\left(\sum_{k=1}^{m}\left(\frac{\partial f_{\psi}}{\partial y^{k}} \frac{\partial \Phi_{k}}{\partial x^{i}}\right)\right)=\sum_{k=1}^{m}\left(\frac{\partial}{\partial x^{j}}\left(\frac{\partial f_{\psi}}{\partial y^{k}} \frac{\partial \Phi_{k}}{\partial x^{i}}\right)\right) \\
& =\sum_{k=1}^{m} \sum_{l=1}^{m}\left(\frac{\partial^{2} f_{\psi}}{\partial y^{l} \partial y^{k}} \frac{\partial \Phi_{l}}{\partial x^{j}} \frac{\partial \Phi_{k}}{\partial x^{i}}+\frac{\partial f_{\psi}}{\partial y^{k}} \frac{\partial^{2} \Phi_{k}}{\partial x^{j} \partial x^{i}}\right) .
\end{aligned}
$$


Since $p$ is a critical point we have that $\frac{\partial f_{\psi}}{\partial y^{k}}(p)=0$ and

$$
\frac{\partial^{2} f_{\varphi}}{\partial x^{i} \partial x^{j}}=\sum_{k, l=1}^{m}\left(\frac{\partial \Phi_{l}}{\partial x^{j}} \frac{\partial^{2} f_{\psi}}{\partial y^{l} \partial y^{k}} \frac{\partial \Phi_{k}}{\partial x^{i}}\right)
$$

Thus the above equation can be written as product of matrices as follows

$$
\left(\frac{\partial^{2} f_{\varphi}}{\partial x^{i} \partial x^{j}}(p)\right)=D \Phi(p)^{T}\left(\frac{\partial^{2} f_{\psi}}{\partial y^{i} \partial y^{j}}(p)\right) D \Phi(p)
$$

where $D \Phi(p)^{T}$ is the transpose of the Jacobian matrix $D \Phi(p)$ of $\Phi$ at $p$. Since $\Phi$ is a diffeomorphism $D \Phi(p)$ is non-singular and therefore $\left(\frac{\partial^{2} f \varphi}{\partial x^{i} \partial x^{j}}(p)\right)$ is non-singular if and only if $\left(\frac{\partial^{2} f_{\psi}}{\partial y^{i} \partial y^{j}}(p)\right)$ is non-singular.

The Lie Bracket of two vector fields $V$ and $W$ on $M$ is the operator $[V, W]: C^{\infty}(M) \rightarrow$ $C^{\infty}(M)$ defined by

$$
[V, W](f)=V(W(f))-W(V(f))
$$

Proposition 1.7. (LEE, 2013, Proposition. 8.26). The Lie bracket of any pair of smooth vector field is a smooth vector field. Moreover, if $\sum_{i=1}^{m} V^{i} \frac{\partial}{\partial x^{i}}$ and $\sum_{i=1}^{m} W^{i} \frac{\partial}{\partial x^{i}}$ are the local coordinate expressions in the chart $\left(U, \phi=\left(x^{1}, \ldots, x^{m}\right)\right)$ of two vector fields $V$ and $W$ in $M$ respectively, then

$$
[V, W]=\sum_{i, j=1}^{m}\left(V^{i} \frac{\partial W^{j}}{\partial x^{i}}-W^{i} \frac{\partial V^{j}}{\partial x^{i}}\right) \frac{\partial}{\partial x^{j}}
$$

Corollary 1.8. Given $p$ a critical point of $f: M \rightarrow \mathbb{R}$, then

$$
[V, W]_{p}(f)=0
$$

for any two vector fields $V, W$ on $M$.

Proof. Since $p$ is a critical point of $f, \frac{\partial f}{\partial x^{i}}(p)=0$ for $i=1, \ldots, m$. Hence, by (1.1) we have that $[V, W]_{p}(f)=0$.

\subsubsection{Morse Index}

Let $p$ be a critical point of the smooth function $f: M \rightarrow \mathbb{R}$. For $v, w \in T_{p} M$ let $V$ and $W$ be two vector fields such that $V_{p}=v$ and $W_{p}=w$. With this, let us define the symmetric bilinear functional $f_{* *}: T_{p} M \times T_{p} M \rightarrow \mathbb{R}$ by $f_{* *}(v, w):=V_{p}(W(f))$, called Hessian of $f$ at $p$.

Proposition 1.9. The Hessian $f_{\star *}(v, w)$ of $f$ at $p$ is symmetric and well defined. 
Proof. Let $[V, W]$ be the Lie bracket, then,

$$
V(W(f))-W(V(f))=[V, W](f)
$$

hence,

$$
V_{p}(W(f))-W_{p}(V(f))=[V, W]_{p}(f) .
$$

Since $p$ is a critical point, by Corollary 1.8 we have that $[V, W]_{p}(f)=0$. Hence, $f_{\star *}(v, w)=$ $f_{\star *}(w, v)$ and the symmetry holds. Moreover, $f_{\star *}$ is well defined i.e., it does not depend of the choice of the extension of $v$ because $f_{* *}(v, w)=v(W(f))$ and does not depend of the choice of the extension of $w$ because $f_{\star *}(v, w)=w(V(f))$.

We will give a local representation of $f_{\star *}$ in a coordinate system.

Let $v=\left.\sum a_{i} \frac{\partial}{\partial x^{i}}\right|_{p}$ and $w=\left.\sum b_{i} \frac{\partial}{\partial x^{i}}\right|_{p}$ be two tangent vectors at $p$. Let $W=\sum b_{i} \frac{\partial}{\partial x^{i}}$ be the extension of $w$ on $U$ where $b_{i}$ is a constant function. Then we have

$$
\begin{aligned}
f_{\star *}(v, w) & =v(W(f))=v\left(\sum_{j} b_{j} \frac{\partial f}{\partial x^{j}}\right) \\
& =\sum_{i, j} a_{i} b_{j} \frac{\partial}{x^{j}}\left(\frac{\partial f}{\partial x^{i}}\right)(p) \\
& =\sum_{i, j} a_{i} b_{j} \frac{\partial^{2} f}{\partial x^{i} \partial x^{j}}(p) .
\end{aligned}
$$

The matrix $H_{p}(f)=\left(\frac{\partial^{2} f}{\partial x^{i} \partial x^{j}}(p)\right)$ represents the bilinear functional $f_{\star *}(v, w)=v^{t} H w$, where $v=\left(a_{i}\right)$ and $w=\left(b_{i}\right)$.

It follows from linear algebra that given $\mathbf{V}$ a finite dimensional vector space and $F$ a bilinear functional on $\mathbf{V}$, the index of $F$ is the dimension of the largest subspace of $\mathbf{V}$ where $F$ is negative definite. The nullity of $F$ on $\mathbf{V}$ is the subspace

$$
\mathbf{W}=\{v \in \mathbf{V} \mid F(v, w)=0 \text { for all } w \in \mathbf{V}\} .
$$

Those definitions can be extended to manifold as follows.

Definition 1.10. The index of $f: M \rightarrow \mathbb{R}$ at a critical point $p$ is the index of the Hessian of $f$ at $p$, which is also called the Morse index of $f$ at $p$.

Proposition 1.11. A point $p \in M$ is a non-degenerate critical point of $f$ if and only if $f_{\star *}$ has nullity equal to zero.

Proof. Denote by $H$ the matrix of $f_{* *}$ in the base $\left(\left.\frac{\partial}{\partial x^{1}}\right|_{p}, \ldots,\left.\frac{\partial}{\partial x^{n}}\right|_{p}\right)$. If $H$ is degenerate then there is $v \in T_{p} M$ such that $v \neq 0$ and $H v=0$, then for all $w \in T_{p} M H(v, w)=w^{t} H v=0$. Therefore, the nullity of $H$ is not zero. If the nullity of $H$ is not zero, then there is non null $v \in T_{p} M$ with $v=\left.\sum a_{i} \frac{\partial}{\partial x^{i}}\right|_{p}$ such that $w^{t} H v=0$ for all $w \in T_{p} M$. In particular, for $w=\left.\frac{\partial}{\partial x^{i}}\right|_{p}$ we have that $w^{t} H v=b_{i}=0$ where $H v=\left(b_{1}, \ldots, b_{n}\right)$. Hence, $H v=0$ and $H$ is singular. 


\subsection{Flows}

Definition 1.12. A 1 - parameter group of diffeomorphism on $M$ (also called smooth global flow on $M$ ) is a smooth map

$$
\theta: \mathbb{R} \times M \longrightarrow M
$$

such that,

(a) The map $\theta_{t}: M \rightarrow M, p \mapsto \theta(t, p)$, is a diffeomorphism for all $t \in \mathbb{R}$,

(b) $\theta_{s+t}=\theta_{s} \circ \theta_{t}$ for all $s, t \in \mathbb{R}$ and $\theta_{0}(p)=p$.

Let $\theta$ be a flow on a smooth manifold $M$. The smooth map $\theta^{p}: \mathbb{R} \rightarrow M, t \mapsto \theta(t, p)$, is called flow line of $\theta$ at $p$. For each global flow $\theta: \mathbb{R} \times M \rightarrow M$ we can associate a vector field $X: M \rightarrow T M, p \mapsto\left(p, \theta_{*}^{p}\left(\left.\frac{d}{d t}\right|_{0}\right)\right)$ which is called the vector field generated by $\theta$. The following proposition guarantees the smoothness of this vector field

Proposition 1.13. (LEE, 2013, Proposition. 9.11). Let $\theta$ a global flow on smooth manifold $M$. Then the vector field $X$ generated by $\theta$ is smooth.

Now, one can ask the inverse process, if we have a smooth vector field $X$ over a manifold $M$, does $X$ generate a smooth global flow on $M$ ? We will give some conditions on the vector field $X$ so that the answer is affirmative. First, we will need the following theorem which is a classic theorem of the ordinary differential equations theory.

Theorem 1.14. (LEE, 2013, Theorem. D.1). Let $U \subset \mathbb{R}^{n}$ and $f: U \rightarrow \mathbb{R}^{n}$ be a smooth map. Consider the initial value problem

$$
\begin{aligned}
\frac{d y}{d t}(t) & =f(y(t)) \\
y\left(t_{0}\right) & =c
\end{aligned}
$$

for arbitrary $t_{0} \in \mathbb{R}$ and $c \in U$.

- Existence: For any $t_{0} \in \mathbb{R}$ and $x_{0} \in U$, there exist an open interval $J_{0}$ with $t_{0} \in J_{0}$ and an open set $U_{0} \subset U$ of $x_{0}$ such that for each $c \in U_{0}$ there is a smooth map $y: J_{0} \rightarrow U$ that solves (1.2) and (1.3).

- Uniqueness: If $y: J \rightarrow U$ and $y^{\prime}: J^{\prime} \rightarrow U$ solves (1.2) and (1.3). Then $\left.y\right|_{J \cap J^{\prime}}=\left.y^{\prime}\right|_{J \cap J^{\prime}}$.

- Smoothness: Let $t_{0}, J_{0}$ and $U_{0}$ as in the Existence statement, and let $\theta: J_{0} \times U_{0} \rightarrow U$ be the map defined by $\theta(t, x)=y(t)$ where $y$ is the unique solution of (1.2) with initial condition $y\left(t_{0}\right)=x$. Then $\theta$ is smooth. 
We say that a smooth vector field $X$ on a smooth manifold $M$ is complete if $X$ is generated by some global flow on $M$.

Lemma 1.15. Let $X$ be a smooth vector field which vanishes outside of a compact set $K \subset M$. Then $X$ is complete.

Proof. It is enough to show that there exists a map $\theta: \mathbb{R} \times M \rightarrow M$ that satisfies (a)-(b) and such that

$$
d_{0} \theta^{p}\left(\left.\frac{d}{d t}\right|_{t=0}\right)=X_{p}, \theta^{p}(0)=p .
$$

Let $p \in M$. Let $\psi=\left(x_{1}, \ldots, x_{n}\right)$ be a local coordinate system in a neighborhood $U$ of $p$. Define $\widetilde{\theta}^{p}=\psi \circ \theta^{p}$. By (1.4) we have

$$
\frac{d \widetilde{\theta^{p}}}{d t}(t)=X^{\prime}\left(\widetilde{\theta^{p}}(t)\right), \widetilde{\theta^{p}}(0)=p^{\prime}
$$

where $X^{\prime}=d \psi(X)$ (i.e., $X^{\prime}(q)=d_{q^{\prime}} \psi\left(X_{q^{\prime}}\right)$ where $q^{\prime}=\psi^{-1}(q)$ ) and $p^{\prime}=\psi(p)$. By Theorem 1.14 we have that for each $(0, p) \in \mathbb{R} \times M$ there are an open interval $J_{p}$ containing 0 and an open subset $U_{p} \subset M$ containing $p$ such that for each $q \in U$ there is a smooth map $\phi^{q}: J_{p} \rightarrow M$ that solves (1.4). Moreover, the map $\phi: J_{p} \times U_{p} \rightarrow M$ defined by $\phi(t, q)=\theta^{q}(t)$ is smooth.

Since $K$ is compact and $K \subset \cup_{p \in K} U_{p}$, there are $p_{1}, \ldots, p_{n} \in K$ such that $K \subset \cup_{i=1}^{n} U_{p_{i}}$. Since $\bigcap_{i=1}^{n} J_{p_{i}}$ is an open interval, there is $\varepsilon>0$ such that $J=(-\varepsilon, \varepsilon) \subset \bigcap_{i=1}^{n} J_{p_{i}}$. Define $\theta: J \times M \rightarrow M$,

$$
\theta(t, p)=\left\{\begin{array}{ccc}
q & \text { if } & q \notin K \\
\phi(t, p) & \text { if } & q \in K
\end{array}\right.
$$

The map $\theta$ is well defined by uniqueness in the previous theorem. By definition we have that $\theta(0, q)=q$. For $s, t, s+t \in J$ we have that $\theta_{t+s}=\theta_{t} \circ \theta_{s}$. Indeed, let $\gamma(t)=\theta_{t+s}(q)$ be a smooth curve, define $l:=\theta_{s}(q)$. Since $\gamma(0)=l=\theta_{0}(l)$, by uniqueness $\theta_{t}(l)=\gamma(t)$ in their common domain. Hence $\theta_{t+s}(q)=\theta_{t}(l)=\theta_{t} \circ \theta_{s}(q)$.

Now, each $t \notin J$ can be express as a multiple of $\varepsilon / 2$ plus a remainder r, i,e., $t=k(\varepsilon / 2)+r$, let $s$ be the sign of $k$, we extend $\theta$ for $t \notin J$ as follows,

$$
\theta(t, p)=\theta_{t}(q)=\theta_{s \varepsilon / 2} \circ \cdots \circ \theta_{s \varepsilon / 2} \circ \theta_{r}
$$

where the map $\theta_{s \varepsilon / 2}$ appears $|k|$ times. It is smooth because is compositions of smooth functions. By construction $\theta_{s+t}=\theta_{s} \circ \theta_{t}$.

Corollary 1.16. On a compact smooth manifold $M$ every smooth vector field is complete.

That gives an answer to our previous question. 


\subsection{Gradient vector field}

In this section we will study the gradient vector field. This vector field is used throughout all the chapters and it is a fundamental tool in the study of Morse Theory. We will give local formulas for this vector field in order to show in Chapter 2 that a non-degenerate critical points of a Morse function $f: M \rightarrow \mathbb{R}$ is a hyperbolic fixed point (see Definition 2.10) of each diffeomophism given by its flow. See Theorem 2.12.

Through this section let $(M, g)$ be a $m$-dimensional compact smooth Riemannian manifold and $f: M \rightarrow \mathbb{R}$ be a smooth function.

Definition 1.17. The gradient vector field $\nabla f$ is the unique smooth vector field such that

$$
g(\nabla f, V)=d f(V)=V(f)
$$

for each tangent vector field $V$ on $M$.

Since $M$ is compact, by Corollary 1.16 there a is local 1-parameter group of diffeomorphisms $\phi: \mathbb{R} \times M \rightarrow M$ generated by $-\nabla f$. Hence

$$
\begin{aligned}
\frac{d}{d t} \phi_{t}(x) & =-\nabla f\left(\phi_{t}(x)\right) \\
\phi_{0}(x) & =x .
\end{aligned}
$$

Proposition 1.18. Let $\left(U,\left(x_{1}, \ldots, x_{m}\right)\right)$ be a local coordinate system of $p$. Then,

- $\left(\left.\frac{\partial}{\partial x_{1}}\right|_{x}, \ldots,\left.\frac{\partial}{\partial x_{m}}\right|_{x}\right)$ span the tangent space $T_{x} U$ for all $x \in U$.

- The component of the metric $g$ are the smooth functions $g_{i j}: U \rightarrow \mathbb{R}$ defined by $g_{i j}(x)=$ $g\left(\left.\frac{\partial}{\partial x_{i}}\right|_{x},\left.\frac{\partial}{\partial x_{j}}\right|_{x}\right)$ for $1 \leq i, j \leq m$. If $V=\sum_{i=1}^{m} V_{i} \frac{\partial}{\partial x_{i}}$ and $W=\sum_{i=1}^{m} W_{i} \frac{\partial}{\partial x_{i}}$ are smooth vector fields on $U$ then

$$
g(V, W)=\sum_{i, j=1}^{m} g_{i j} V_{i} W_{i}
$$

- Since $g$ is non-degenerate, the matrix $\left(g_{i j}(x)\right)$ has inverse for all $x \in U$ which we denote $\left(g^{i j}(x)\right)$.

- $\left(d_{x_{1}} \ldots, d_{x_{m}}\right)$ is a basis for the cotangent bundle $T^{\star} U$, where $d_{x_{i}}: T U \rightarrow \mathbb{R}$ is defined $d_{x_{i}}\left(\frac{\partial}{\partial x_{j}}\right)=\delta_{i j}$ (Kronecker delta), and

$$
d f=\sum_{i=0}^{m} \frac{\partial f}{\partial x_{i}} d_{x_{i}}
$$

The following Lemma give us a local description of the gradient vector field. 
Lemma 1.19. Let $(M, g)$ a Riemannian manifold and $f: M \rightarrow \mathbb{R}$ be a smooth function. Then in a local coordinate system the following holds,

$$
\nabla f=\sum_{i, j=1}^{m} g^{i j} \frac{\partial f}{\partial x_{i}} \frac{\partial}{\partial x_{j}} .
$$

Proof. In the local coordinate system $\left(U,\left(x_{1}, \ldots, x_{n}\right)\right)$ suppose that $\nabla f=\sum_{i=1}^{m} V_{j} \frac{\partial}{\partial x_{i}}$, then for $i=1, \ldots, m$ we have

$$
\begin{aligned}
\frac{\partial f}{\partial x_{j}} & =g\left(\nabla f, \frac{\partial}{\partial x_{j}}\right) \\
& =\sum_{i=0}^{m} g_{i j} V_{j} \\
& =\left(V_{1}, \ldots, V_{m}\right)\left(g_{1 j}, \ldots, g_{m j}\right)^{\prime} .
\end{aligned}
$$

Therefore,

$$
\left(V_{1}, \ldots, V_{m}\right)\left(g_{i j}\right)=\left(\frac{\partial f}{\partial x_{1}}, \ldots, \frac{\partial f}{\partial x_{m}}\right)
$$

and since $\left(g^{i j}\right)=\left(g_{i j}\right)^{-1}$,

$$
\left(V_{1}, \ldots, V_{m}\right)=\left(\frac{\partial f}{\partial x_{1}}, \ldots, \frac{\partial f}{\partial x_{m}}\right)\left(g^{i j}\right)
$$

so,

$$
V_{j}=\sum_{i=1}^{m} g^{i j} \frac{\partial f}{\partial x_{i}}
$$

Lemma 1.20. Suppose further that $\frac{\partial}{\partial x_{1}}, \ldots, \frac{\partial}{\partial x_{m}}$ is an orthonormal basis for $T_{p} U$ with respect to the metric $g$. Then the matrix representation for the differential of $\nabla f: U \rightarrow U \times \mathbb{R}^{m}$ is equal to the Hessian matrix at $p$.

Proof. By previous Lemma we have that

$$
\nabla f=\left(\sum_{k=1}^{m} g^{k 1} \frac{\partial f}{\partial x_{k}}, \ldots, \sum_{k=1}^{m} g^{k m} \frac{\partial f}{\partial x_{k}}\right) .
$$

Now, we will compute the matrix of $d \nabla f_{p}: T_{p} U \rightarrow T_{q} \mathbb{R}^{m}$, where $q=\nabla f(p)$,

$$
\begin{aligned}
d_{p} \nabla f\left(\left.\frac{\partial}{\partial x_{i}}\right|_{p}\right) & =\left.\left.\sum_{j=1}^{m} \frac{\partial}{\partial x_{i}}\right|_{p}\left(\sum_{k=1}^{m} g^{k j} \frac{\partial f}{\partial x_{k}}\right) \frac{\partial}{\partial x_{j}}\right|_{q} \\
& =\left.\sum_{j=1}^{m}\left(\sum_{k=1}^{m} \frac{\partial g^{k j}}{\partial x_{i}}(p) \frac{\partial f}{\partial x_{k}}(p)+g^{k j}(p) \frac{\partial f}{\partial x_{i} x_{k}}(p)\right) \frac{\partial}{\partial x_{j}}\right|_{q} \\
& =\left.\sum_{j=1}^{m} \frac{\partial f}{\partial x_{i} x_{k}}(p) \frac{\partial}{\partial x_{j}}\right|_{q}
\end{aligned}
$$


since $\frac{\partial}{\partial x_{1}}, \ldots, \frac{\partial}{\partial x_{m}}$ is an orthonormal basis and $p$ is a critical point, the last equality holds.

Hence, the matrix representation of $d_{p} \nabla f: T_{p} U \rightarrow T_{p} U \times T_{p} \mathbb{R}^{m}$ is equal to Hessian matrix of $f$,

$$
d_{p} \nabla f=\left(I_{T_{p} U}, H_{p}(f)\right)=\left(\frac{\partial^{2} f}{\partial x_{i} x_{j}}(p)\right)_{i j} .
$$

Example 1.21. Let $r: \mathbb{R}^{3} \rightarrow \mathbb{R}$ the height function, i.e, $r(x, y, z)=z$. Consider the Euclidean metric on $\mathbb{R}^{3}$. We will compute the gradient vector field of the restriction $h=\left.r\right|_{S^{2}}: S^{2} \rightarrow \mathbb{R}$. Note that the vector $\nabla h(x, y, z)$ is the projection onto the tangent space of $S^{2}$ at $(x, y, z)$ of the vector $\nabla r(x, y, z)$, see Figure 1 .

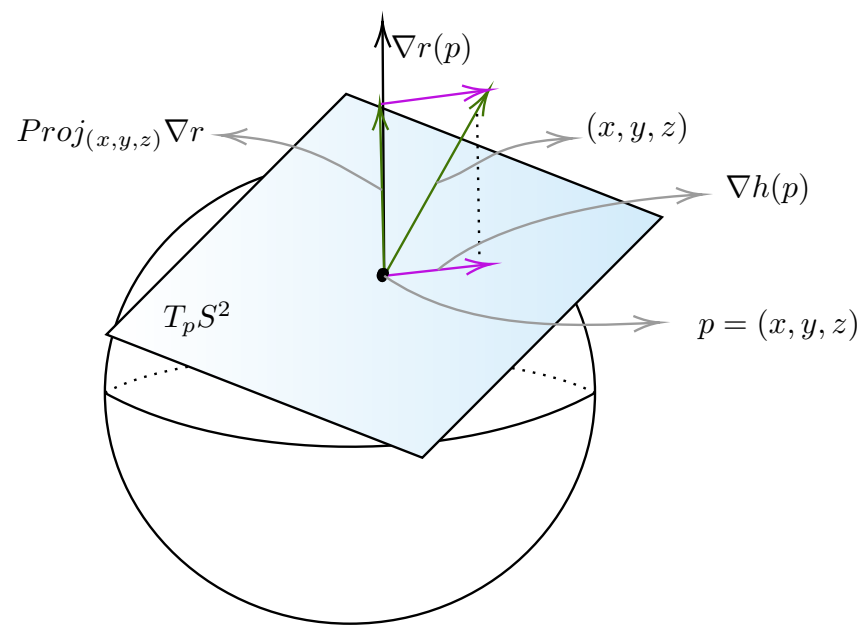

Figure 1 - Projected vector field

$$
\begin{aligned}
\nabla h(x, y, z) & =\nabla r-\operatorname{Proj}_{(x, y, z)}(\nabla r) \\
& =\nabla r-\left\langle\nabla r, \frac{(x, y, z)}{\|(x, y, z)\|}\right\rangle \frac{(x, y, z)}{\|(x, y, z)\|} \\
& =(0,0,1)-\langle(0,0,1),(x, y, z)\rangle(x, y, z) \\
& =\left(-x z,-y z, 1-z^{2}\right)
\end{aligned}
$$

In Figure 2 we see some flow lines of $\nabla h$ on $S^{2}$.

Example 1.22. Consider the torus as the quotient $T^{2}=\mathbb{R}^{2} / \mathbb{Z}^{2}$. Define the function

$$
\begin{aligned}
f: T^{2} & \longrightarrow \mathbb{R} \\
(x, y) & \longmapsto \cos (2 \pi x)+\cos (2 \pi y) .
\end{aligned}
$$

Then the gradient vector field is

$$
\begin{aligned}
\nabla f: T^{2} & \longrightarrow \mathbb{R}^{2} \\
(x, y) & \longmapsto(-2 \pi \sin (2 \pi x),-2 \pi \sin (2 \pi y))
\end{aligned}
$$




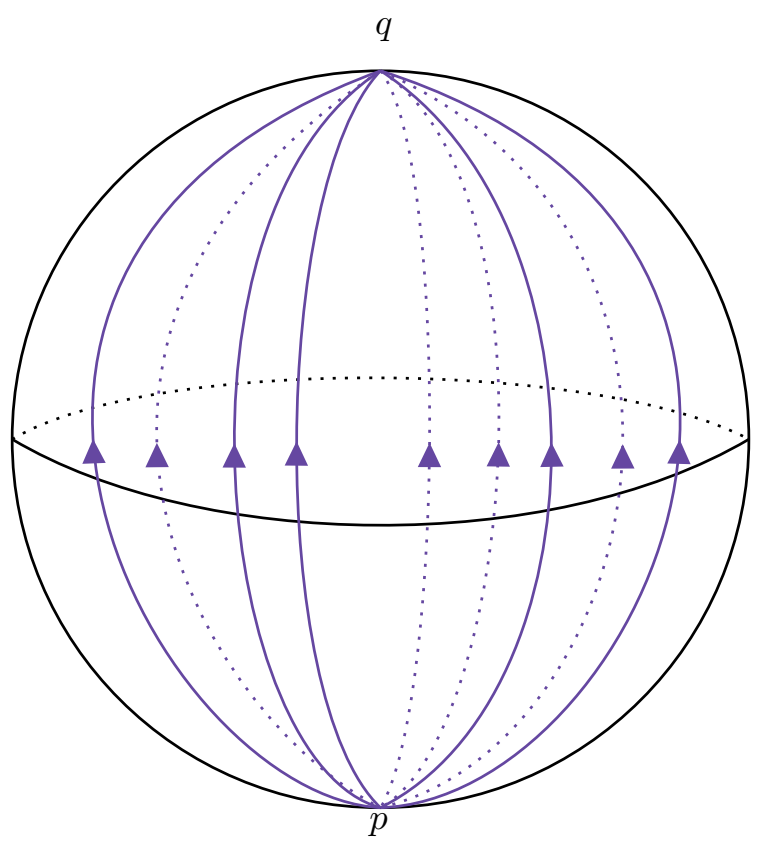

Figure 2 - Flow lines of $\nabla h$ on $S^{2}$

In Figure 3 we see the vector field and the flow lines. The points $p=(1 / 2,1 / 2), q=(1 / 2,0), r=$ $(0,1 / 2)$ and $s=(0,0)$ are critical point of $f$ where $s$ is a maximal point and $p$ is a minimal point $f$. In Figure 4 we see the classical representation of the torus and the position of the points $p, q, r$ and $s$ with some flow lines of $\nabla f$

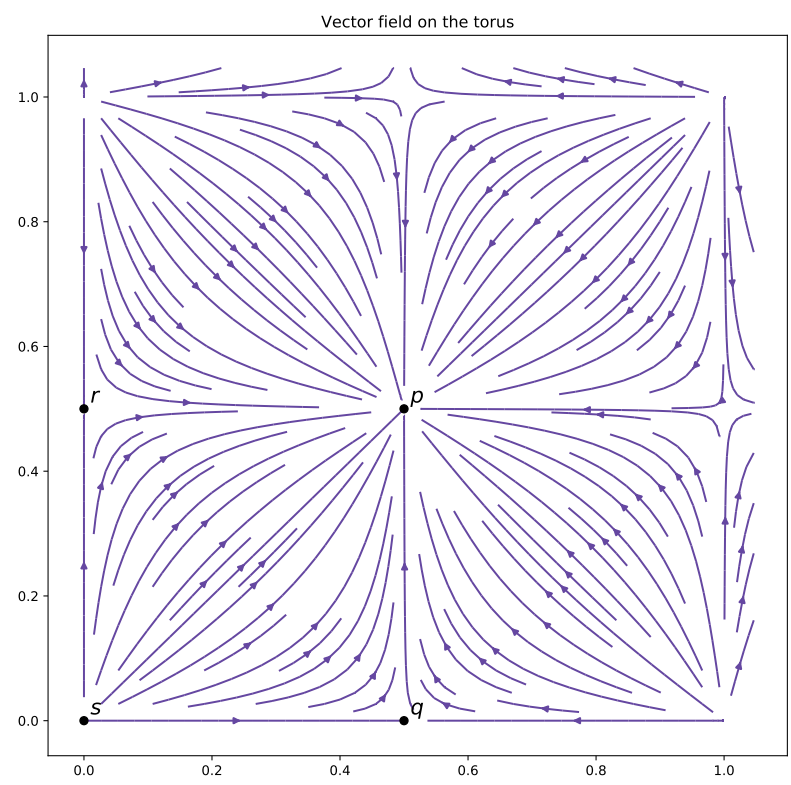

Figure 3 - Vector field on $\mathbb{R}^{2} / \mathbb{Z}^{2}$ 


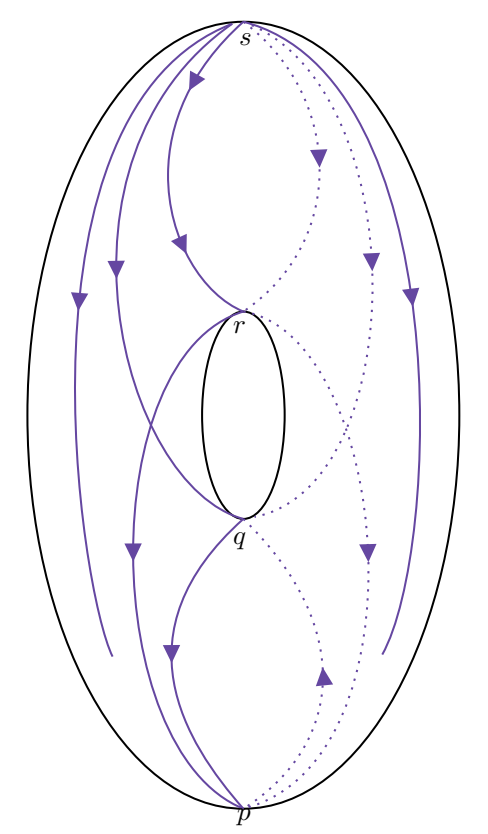

Figure 4 - Vector field on the torus

For the following lemma we need the concept the Matrix exponential, for more details (BELLMAN, 1997).

Definition 1.23. Let $A$ be a $n \times n$ real matrix, we define the exponential matrix:

$$
e^{A}=\sum_{k=0}^{\infty} \frac{1}{k !} A^{k} .
$$

In particular, if $A$ is a diagonal matrix $\operatorname{diag}\left(a_{1}, \ldots, a_{n}\right)$ then $e^{A}=\operatorname{diag}\left(e^{a_{1}}, \ldots, e^{a_{n}}\right)$.

Lemma 1.24. Let $\left(U,\left(x_{1}, \ldots, x_{m}\right)\right)$ be a local chart around the critical point $p$ of a smooth function $f$. Suppose that $\frac{\partial}{\partial x_{1}}, \ldots, \frac{\partial}{\partial x_{m}}$ is an orthonormal basis for $T_{p} U$. Then, for each fixed $t \in \mathbb{R}$ we have that

$$
d_{p}\left(\phi_{t}\right)=e^{-H_{p}(f) t}
$$

Proof. By existence and uniqueness of Theorem 1.14, we have that for some $\varepsilon>0$ and neighborhood $U$ of $p$ there is a unique smooth map $\phi:(-\varepsilon, \varepsilon) \times U \rightarrow U$ such that $\phi(0, x)=x$ and

$$
\frac{d}{d t} \phi(t, x)=-\nabla f(\phi(t, x)) .
$$

Then,

$$
\begin{aligned}
d_{x}\left(\frac{d}{d t} \phi(t, x)\right) & =-d_{x}(\nabla f(\phi(t, x))) \\
\frac{d}{d t}\left(d_{x} \phi(t, x)\right) & =-\left(d_{\phi(t, x)} \nabla f\right)\left(d_{x} \phi(t, x)\right)
\end{aligned}
$$


Therefore $d_{x} \phi(x, t)$ is the solution of the following linear system of EDOs,

$$
\begin{aligned}
\frac{d}{d t}(\Phi(t, x)) & =-\left(d_{\phi(t, x)} \nabla f\right)(\Phi(t, x)) \\
\Phi(0, x) & =I d
\end{aligned}
$$

and according to (BELLMAN, 1997, pag. 167) another solution for the system is

$$
\Phi(t, x)=e^{-\left(d_{x} \nabla f\right) t} .
$$

Then by Lemma 1.20 we have that $e^{-\left(d_{x} \nabla f\right) t}=e^{-\left(H_{p}(f)\right) t}$. Hence, by uniqueness we conclude that

$$
d_{x} \phi(x, t)=e^{-\left(H_{p}(f)\right) t}
$$

\subsection{Singular Homology}

In this section we define the singular homology and present their fundamental properties. For more details see (GREENBERG, 1967), (HATCHER, 2002).

Let $k \in \mathbb{N}$. The standard $k$-simplex is the subspace of $\mathbb{R}^{k+1}$,

$$
\Delta^{k}=\left\{\left(t_{0}, \ldots, t_{k}\right) \in \mathbb{R}^{k+1} \mid t_{i} \geq 0 \text { and } t_{0}+t_{1}+\cdots+t_{k}=1\right\} .
$$

For $0 \leq i \leq k$ define the face maps $F_{i}^{k}: \Delta^{k-1} \rightarrow \Delta^{k}$ by

$$
F_{i}^{k}\left(t_{0}, \ldots, t_{k-1}\right)=\left(t_{0}, \ldots, t_{i-1}, 0, t_{i}, \ldots, t_{k-1}\right) \text {. }
$$

A singular $k$-simplex in a space $X$ is a continuous map $\sigma: \Delta^{k} \rightarrow X$. Let $C_{k}(X ; \mathbb{Z})$ be the free $\mathbb{Z}$-module with generators the singular $k$-simplices in $X$, i.e., an element of $C_{k}(X ; \mathbb{Z})$ is a finite formal sum $\sum_{i \in I} \lambda_{i} \sigma_{i}$ where $\lambda_{i} \in \mathbb{Z}$ and $\sigma_{i}$ are $k$-simplices. There is a boundary operator

$$
\partial_{k}: C_{k}(X ; \mathbb{Z}) \rightarrow C_{k-1}(X ; \mathbb{Z})
$$

defined on a generator $\sigma$ by

$$
\partial_{k}(\sigma)=\sigma \circ F_{0}^{k}-\sigma \circ F_{1}^{k}+\cdots+(-1)^{k} \sigma F_{k}^{k}
$$

and extending by linearity, $\partial_{k}\left(\sum_{i \in I} \lambda_{i} \sigma_{i}\right)=\sum_{i \in I} \lambda_{i} \partial_{k}\left(\sigma_{i}\right)$.

This boundary operator satisfies $\partial_{k-1} \circ \partial_{k}=0$. Then the image of $\partial_{k}$, which is denoted by $B_{k}(X ; \mathbb{Z})$ is contained in the kernel of $\partial_{k-1}$ which is denoted by $Z_{k}(X ; \mathbb{Z})$.

Definition 1.25. The quotient $\mathbb{Z}$-module

$$
H(X ; \mathbb{Z})=\frac{Z_{k}(X ; \mathbb{Z})}{B_{k}(X ; \mathbb{Z})}
$$

is called the $k^{\text {th }}$ singular homology group of $X$ with coefficients in $\mathbb{Z}$. 
Let $A \subset X$ be a subspace of $X$, the pair $(X, A)$ is called topological pair. Clearly $C_{k}(A ; \mathbb{Z})$ is a submodule of $C_{k}(X ; \mathbb{Z})$. We denote the $\mathbb{Z}$-module quotient by

$$
C(X, A ; \mathbb{Z})=\frac{C_{k}(X ; \mathbb{Z})}{C_{k}(A ; \mathbb{Z})}
$$

The boundary operator $\partial_{k}$ induces a well defined a homomorphism between the quotient modules

$$
\bar{\partial}_{k}: C_{k}(X, A ; \mathbb{Z}) \rightarrow C_{k-1}(X, A ; \mathbb{Z})
$$

because $\partial_{k}\left(C_{k}(A ; \mathbb{Z})\right) \subset C_{k-1}(A ; \mathbb{Z})$ and $\bar{\partial}$ also satisfies $\bar{\partial}_{k-1} \circ \bar{\partial}_{k}=0$. Denote $B_{k}(X, A ; \mathbb{Z})=$ $\operatorname{Im}\left(\partial_{k+1}\right)$ and $Z_{k}(X, A ; \mathbb{Z})=\operatorname{ker}\left(\partial_{k}\right)$.

Definition 1.26. The quotient $\mathbb{Z}$-module

$$
H_{k}(X, A ; \mathbb{Z})=\frac{Z_{k}(X, A ; \mathbb{Z})}{B_{k}(X, A ; \mathbb{Z})}
$$

is called the $k^{\text {th }}$ relative homology group of $X$ with coefficients in $\mathbb{Z}$.

In order to simplify notation, we denote $H(X ; \mathbb{Z})$ and $H(X, A ; \mathbb{Z})$ by $H(X)$ and $H(X, A)$ respectively. By definition, a map $f:(X, A) \rightarrow(Y, B)$ between topological pairs is a continuous function $f: X \rightarrow Y$ such that $f(A) \subset B$. The function $f$ induces a $\mathbb{Z}$-homomorphism $f_{\star}: H_{\star}(X, A) \rightarrow H_{\star}(Y, B), f_{\star}(\bar{\sigma})=\overline{f \circ \sigma}$.

The induced $\mathbb{Z}$-homomorphism is functoriality, i.e., if $g:(Y, B) \rightarrow(Z, C)$ is a map of pairs, then $(g \circ f)_{*}=g_{*} \circ f_{\star}$.

Definition 1.27. Let $f, g:(X, A) \rightarrow(Y, B)$ a map of pair. We say that $f$ and $g$ are homotopic, which is denoted $f \simeq g$, if there exists a continuous map $H: X \times I \rightarrow Y$ such that

- $H(x, 0)=f(x)$ for all $x \in X$,

- $H(x, 1)=g(x)$ for all $x \in X$,

- $H(x, t) \in B$ for all $x \in A$ and $t \in I$.

Definition 1.28. A map $f:(X, A) \rightarrow(Y, B)$ is called homotopy equivalence if there is a map $g:(Y, B) \rightarrow(X, A)$ such that $g \circ f \simeq I d_{X}$ and $f \circ g \simeq I d_{Y}$, where $I d_{X}$ and $I d_{Y}$ are the identity maps on $X$ and $Y$, respectively. We say that $(X, A)$ and $(Y, B)$ have the same homotopy type, which we denoted by $(X, A) \simeq(Y, B)$, if there is a homotopy equivalence $f:(X, A) \rightarrow(Y, B)$.

Definition 1.29. Given a topological pair $(X, A)$ we say that $A$ is a deformation retract of $X$ if there is a homotopy $h: I \times X \rightarrow X$ such that $h(t, x)=x$ if $x \in A$ or $t=0$ and $h(1, X)=A$.

Remark 1.30. Note that if $A$ is a deformation retract of $X$ then the inclusion map $i_{A}: A \hookrightarrow X$ is a homotopy equivalence since $h_{1} \circ i_{A}=I d_{A}$ where $h_{1}=h(1, \bullet)$ and $i_{A} \circ h_{1}$ is homotopic to $I d_{X}$ through the homotopy $H$ where $H(t, x)=h(1-t, x)$. In fact,

$$
H(0, x)=h(1, x)=h_{1}(x)=i_{A} \circ h_{1}(x) \text { and } H(1, x)=h(0, x)=I d_{X} .
$$


Now, we present a fundamental properties of the singular homology groups.

Proposition 1.31. Let $(X, A)$ and $(Y, B)$ be a topological pairs. Then we have the following properties.

1. (Homotopy property.) Let $f, g:(X, A) \rightarrow(Y, B)$ be two homotopic continuous functions then $f_{\star}=g_{*}$. From that it follows that if $(X, A) \simeq(Y, B)$ then $H_{*}(X, A) \approx H_{*}(Y, B)$.

2. (Exactness property.) There is connecting homomorphism

$$
\partial_{k}: H_{k}(X, A) \rightarrow H_{k-1}(A), \bar{\sigma} \mapsto \overline{\partial_{k}(\sigma)},
$$

such that the following sequence is exact

$$
\cdots \rightarrow H_{k}(A) \rightarrow H_{k}(X, A) \rightarrow H_{k-1}(A) \rightarrow \cdots
$$

Moreover, $\partial_{k}$ is a natural transformation, i.e., the following diagram is commutative

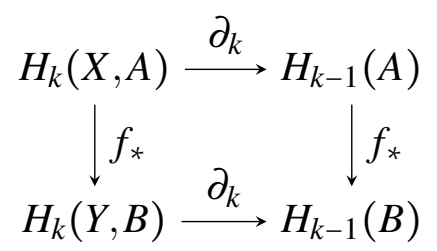

3. (Excision property.) Let $U \subset A \subset X$ with $\bar{U} \subset \operatorname{int}(A)$, then we have the isomorphism

$$
H_{*}(X, A) \approx H_{*}(X-U, A-U) .
$$

4. (Dimension property.) Let $*$ be a space of one point, then

$$
H_{k}(*)= \begin{cases}\mathbb{Z} & \text { if } k=0 \\ 0 & \text { if } k \neq 0 .\end{cases}
$$

5. (Additivity property.) Let $X=\sqcup_{\alpha} X_{\alpha}$, then

$$
H_{*}(X) \approx \bigoplus_{\alpha} H_{k}\left(X_{\alpha}\right)
$$

\subsection{CW-Complexes}

The CW-complexes are central concept in the study of Morse theory. For instance, given a smooth manifold $M$, one of the main Theorem (see Theorem 2.21) states that $M$ has the same homotopy type of a CW-complex. The CW-homology Theorem that we present in this section (see Theorem 1.38) is used in the proof of Morse inequalities (see Theorem 2.23).

Definition 1.32. The topological space $e^{k}=\left\{x \in \mathbb{R}^{k} \mid\|x\| \leq 1\right\}$ with boundary $\partial e^{k}=S^{k-1}=\{x \in$ $\left.\mathbb{R}^{k} \mid\|x\|=1\right\}$ is called $k$-cell. 
Let $Y$ be a topological space and let $g: S^{k-1} \rightarrow Y$ be a continuous function. The topological space $Y \cup_{g} e^{k}$ ( $Y$ attaching a $k$-cell) is defined to be the quotient space $Y \sqcup e^{k} / \sim$ where $a \sim b$, if and only if, $b=g(a)$ or $a=g(b)$ or $g(a)=g(b)$.The function $g$ called attaching map.

Definition 1.33 (CW-Complex). A topological space $X$ has the structure of $C W$-complex if there exists a chain of subspaces that satisfies

$$
X^{0} \subset X^{1} \subset \cdots \subset X=\bigcup_{n \in \mathbb{Z}_{+}} X^{n}
$$

and such that,

1. $X^{0}$ is a discrete set of points, 0 -cells.

2. $X^{n}$ is obtain from $X^{n-1}$ by attaching $n$-cells.

3. $X=\bigcup_{n} X^{n}$ has the weak topology, i.e., the set $U \subset X$ is open iff $U \cap X^{n}$ is open in $X^{n}$ for each $n \in \mathbb{Z}_{+}$.

$X^{n}$ is called $n$-skeleton. $X$ is a CW-complex finite if the chain is finite, i.e., $X=X^{n}$ for some $n \in \mathbb{Z}_{+}$.

Let $X$ be a topological space and $A \subset X$ be a non empty closed subspace, we say that $(X, A)$ is a good pair if $A$ is a deformation retract of some neighborhood in $X$. A topological pair that is good pairs has a useful property. That is, there is an isomorphism between the relative homology of the pair with the homology of the quotient. See Theorem 1.35.

Lemma 1.34. Let $X$ be a CW-complex and $A$ be a subcomplex of $X$. Then $(X, A)$ is a good pair.

Proof. We will construct a neighborhood of $N(A)$ of $A$ inductively over the skeleton $X^{n}$ :

- For $n=0 . N^{0}=A \cap X^{0}$.

- Suppose that we have $N^{n}(A)$ a neighborhood of $A \cap X^{n}$ in $X^{n}$.

- Now the inductive step. Let $\phi_{\alpha}: D_{\alpha}^{n+1} \rightarrow X$ be the set of all characteristic maps of $n$-cells of $X$. Let $0<\varepsilon<1$. Let $V_{1}^{\prime}$ be is an open neighborhood of $\phi_{\alpha}^{-1}(A)-\partial D_{\alpha}^{n+1} \operatorname{in} \operatorname{Int}\left(D^{n+1}\right)$. Consider the spherical coordinates $(r, \theta)$ in $D^{n+1}$ where $r \in[0,1]$ and $\theta \in \partial D^{n+1}$. Let $V_{2}^{\prime}=(1-\varepsilon, 1] \times \phi_{\alpha}^{-1}\left(N^{n}(A)\right) \subset D_{\alpha}^{n+1}$. Define $V_{1}=\phi_{\alpha}\left(V_{1}^{\prime}\right)$ and $V_{2}=\phi_{\alpha}\left(V_{2}^{\prime}\right)$. Let us define $N^{n}(A)=\cup_{\alpha} N_{\alpha}^{n}(A)$ where $N_{\alpha}^{n+1}(A)=V_{1} \cup V_{2}$. Clearly each $N_{\alpha}^{n}(A)$ is open because $V_{1}$ and $V_{2}$ are open.

- Then we define $N(A)=\bigcup_{n} N^{n}(A)$. 
It remains to show that $A$ is a deformation retract of $N(A)$. In each cell $\phi_{\alpha}: D_{\alpha}^{k} \rightarrow X$ of $X-A$, $N(A)$ is a product of $(1-\varepsilon, 1] \times \partial D_{\alpha}^{k}$ and it is a deformation retracts onto $\partial D^{k}$ through $r^{\prime}(s, x)=$ $(s(1-t)+t, x)$. Let $r: I \times N(A) \rightarrow N(A)$ defined by $r(t, x)=\phi_{\alpha} \circ r_{t}^{\prime} \circ \phi^{-1}(x)$ if $x \in \operatorname{Im} \phi_{\alpha}$ and $r(t, x)=x$ if $x \in A$. Then $r$ is a deformation retract of $N(A)$ onto $A$.

In a topological pair $(X, A)$ consider the equivalence relation $\sim$ as on $X$ as follows: If $a, b \in A$ then $a \sim b$, if $x \notin A$ then $[x]=\{x\}$. Let $X / \sim$ be the set of equivalence classes that we denote by $X / A$ and let $f: X \rightarrow X / A$ be the quotient map. The $X / A$ is a topological space where $U \subset X / A$ is open if and only if $f^{-1}(U)$ is open in $X$. The space $X / A$ is called quotient topological space.

Theorem 1.35. Let $X$ be a topological space and $A \subset X$ be a non empty closed subspace. Suppose that $(X, A)$ is a good pair. Then $H_{k}(X, A) \approx H_{k}(X / A, *)$ for all $k \geq 0$ where $*$ is the space of a point. Hence, for $k>0$ we have that $H_{k}(X, A) \approx H_{k}(X / A)$.

Proof. Let $V$ be the a neighborhood of $A$ which is a deformation retract of $A$. Let $q:(X, A) \rightarrow$ $(X / A, A / A)$ be the quotient map. We have a commutative diagram

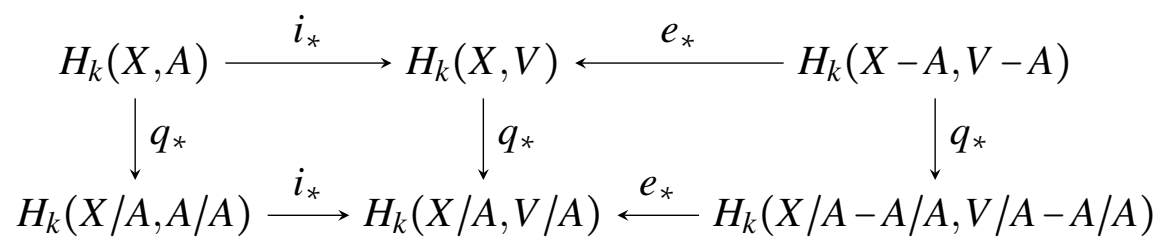

where, $i$ and $e$ are the inclusions. The long sequence of the triple $(X, V, A)$ is

$$
\cdots \rightarrow H_{k}(V, A) \rightarrow H_{k}(X, A) \rightarrow H_{k}(X, V) \rightarrow H_{k-1}(V, A) \rightarrow \cdots
$$

Since $(V, A) \simeq(A, A), H_{k}(V, A) \approx H_{k}(A, A)=0$. Hence, the upper $i_{\star}$ is an isomorphism. A deformation retract of $V$ onto $A$ gives a deformation retract of $V / A$ onto $A / A$ and by the same argument the lower $i_{\star}$ is an isomorphism. The $e_{*}$ are isomorphism by excision. Note that $q:(X-A, V-A) \rightarrow(X / A-A / A, V / A-A-A)$ is an homeomorphism, then the right map $q_{*}$ is an isomorphism, therefore $H(X, A) \approx H_{k}(X / A, A / A)$.

The following corollary follows directly from the Theorem 1.35 and Lemma 1.34.

Corollary 1.36. Let $X$ be a CW-complex and let $A$ be a subcomplex of $X$. Then $H_{k}(X, A)=$ $H_{k}(X / A, *)$ for all $k \geq 0$.

Now, we give some properties of the homology of the $C W$-complexes.

Lemma 1.37. Let $X$ be a CW complex. Then the following holds. 
1.

$$
H_{k}\left(X^{n}, X^{n-1}\right) \approx\left\{\begin{array}{cll}
0 & \text { if } k \neq n, \\
\bigoplus_{\alpha \in J} \mathbb{Z} & \text { if } k=n,
\end{array}\right.
$$

where $J$ is in one-to-one correspondence with the $n$-cells of $X$.

2. $H_{k}\left(X^{n}\right)=0$ for $k>n$. Then, if $X$ is finite we have that $H_{k}(X)=0$ for $k>\operatorname{dim} X$.

3. The map $i_{\star}: H_{k}\left(X^{n}\right) \rightarrow H_{k}(X)$ induced by the inclusion $i: X^{n} \hookrightarrow X$ is an isomorphism for $k<n$ and surjective for $k=n$.

Proof. 1. We have that $X^{n} / X^{n-1}=\bigvee_{\alpha \in J} S^{n}$. Let $x_{0} \in S^{n}$ be the south pole. Since $\left(\sqcup_{\alpha} S^{n}, \sqcup_{\alpha} x_{0}\right)$ is a good pair, $H_{k}\left(\sqcup_{\alpha} S^{n}, \sqcup_{\alpha} x_{0}\right) \approx H_{k}\left(\bigvee_{\alpha \in J} S^{n}, x_{0}\right)$. Since $H_{k}\left(x_{0}\right)=0$, from the long exact sequence of the pair $\left(\sqcup_{\alpha} S^{n}, \sqcup_{\alpha} x_{0}\right)$

$$
0=H_{k}\left(\sqcup_{\alpha} x_{0}\right) \rightarrow H_{k}\left(\sqcup_{\alpha} S^{n}\right) \rightarrow H_{k}\left(\sqcup_{\alpha} S^{n}, \sqcup_{\alpha} x_{0}\right) \rightarrow H_{k-1}\left(\sqcup_{\alpha} x_{0}\right)=0
$$

we have that

$$
H_{k}\left(\sqcup_{\alpha} S^{n}\right) \approx H_{k}\left(\sqcup_{\alpha} S^{n}, \sqcup_{\alpha} x_{0}\right) .
$$

By the aditivity property

$$
H_{k}\left(\sqcup_{\alpha \in J} S^{n}\right) \approx \bigoplus_{\alpha} H_{k}\left(S^{n}\right)
$$

2. Consider the long exact sequence of the pair $\left(X^{n}, X^{n-1}\right)$,

$$
\cdots \rightarrow H_{k+1}\left(X^{n}, X^{n-1}\right) \rightarrow H_{k}\left(X^{n-1}\right) \rightarrow H_{k}\left(X^{n}\right) \rightarrow H_{k}\left(X^{n}, X^{n-1}\right) \rightarrow \cdots
$$

by (1), if $k \neq n, H_{k}\left(X^{n}, X^{n-1}\right)=0$, then $H_{k}\left(X^{n-1}\right) \rightarrow H_{k}\left(X^{n}\right)$ is surjective. If $k+1 \neq n$, then it is injective. Since $H_{k}\left(X^{0}\right)=0$ for $k>0, H_{k}\left(X^{n}\right)=0$ for $k>n$.

3. When $k<n$ we have that $H_{k}\left(X^{n}\right) \rightarrow H_{k}\left(X^{n+1}\right)$ is an isomorphism by the previous item. If $X$ is finite it is clear that $H_{k}\left(X^{n}\right) \rightarrow H_{k}(X)$ is an isomorphism because $X=X^{m}$ for some $m \in \mathbb{Z}_{+}$. 
of the pairs $\left(X^{n+1}, X^{n}\right),\left(X^{n}, X^{n-1}\right)$ and $\left(X^{n-1}, X^{n-2}\right)$,

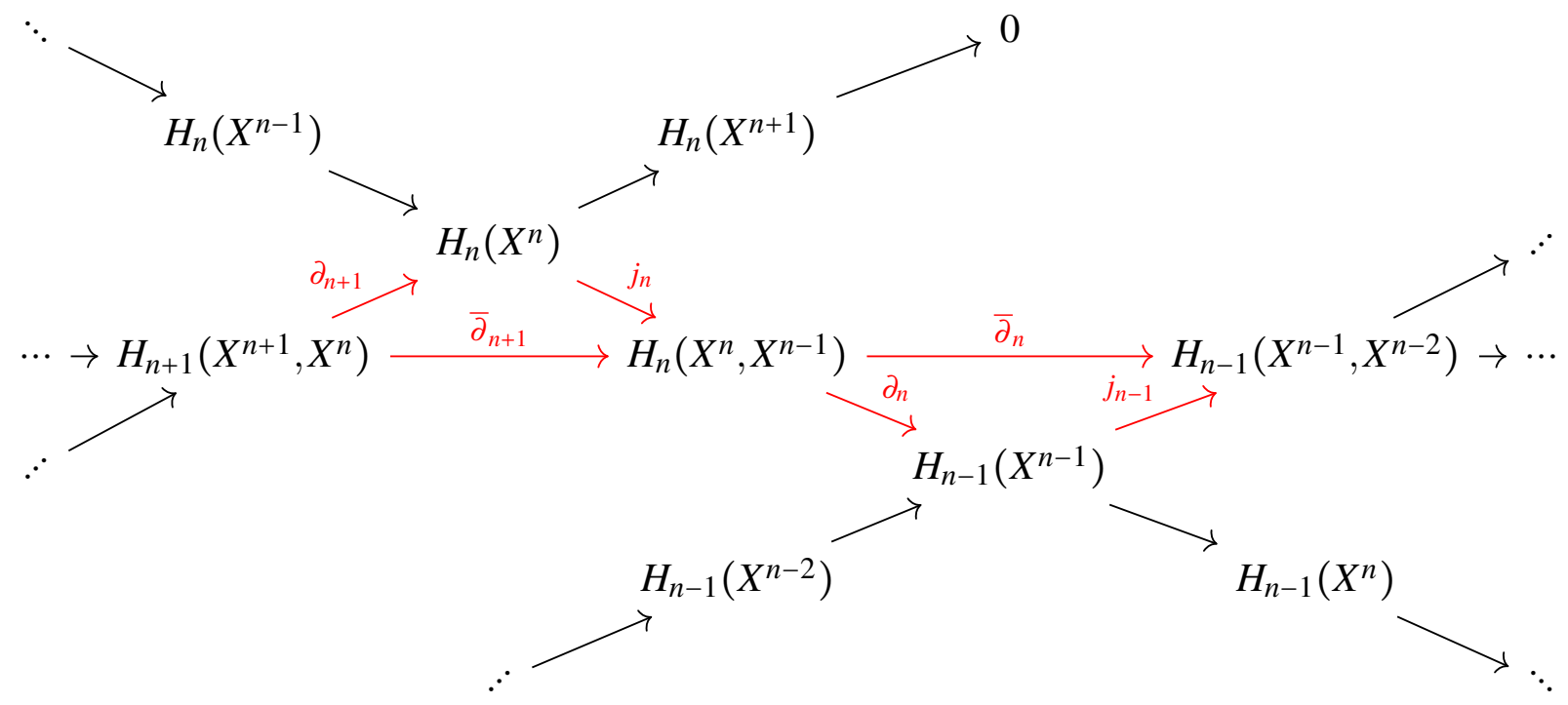

Define $\bar{\partial}_{n+1}=j_{n} \circ \partial_{n+1}$. Note that $\left(H_{n}\left(X^{n}, X^{n-1}\right), \bar{\partial}_{n}\right)$ is a chain complex, clearly $\bar{\partial} \circ \bar{\partial}=0$.

Theorem 1.38 (CW homology Theorem). (HATCHER, 2002, Theorem. 2.35) The homology modules of the chain complex $\left(H_{n}\left(X^{n}, X^{n-1}\right), \bar{\partial}_{n}\right)$ are isomorphic to the singular homology modules of $X$, i.e.,

$$
H_{n}(X) \approx \frac{\operatorname{ker}\left(\overline{\partial_{n}}\right)}{\operatorname{Im}\left(\bar{\partial}_{n+1}\right)} .
$$

The boundary operator $\bar{\partial}_{n}$ in this case is given by a formula that we will explore in more details in the examples ahead.

By Lemma 1.37 we know that $H_{n}\left(X^{n}, X^{n-1}\right)$ is generated by the $n$-cells $e_{\alpha}^{n}$ of $X$. Then $\bar{\partial}_{n}\left(e_{\alpha}^{n}\right)=\sum_{\beta} d_{\alpha \beta} e_{\beta}^{n-1}$ where $\left\{e_{\beta}^{n-1}\right\}_{\beta}$ are the generators of $H_{n-1}\left(X^{n-1}, X^{n-2}\right)$ and $d_{\alpha \beta}$ is the degree of the map $\pi_{\beta} \circ f_{\alpha}$

$$
S_{\alpha}^{n-1} \stackrel{f_{\alpha}}{\longrightarrow} X^{n-1} \stackrel{\pi_{\beta}}{\longrightarrow} X^{n-1} /\left(X^{n-1}-e_{\beta}^{n-1}\right) \approx S_{\beta}^{n-1},
$$

where $f_{\alpha}$ is the attaching map of $e_{\alpha}^{n}$ and $\pi_{\beta}$ is quotient map collapsing the space $X^{n-1}-e_{\beta}^{n-1}$ to a point.

Example 1.39. The $n$-sphere has the structure of CW-complex. Indeed, consider de map $g$ : $\partial e^{n} \rightarrow e^{0}$, let $X^{0}=e^{0}$, then $S^{n}=X^{n}=X^{0} \cup_{g} e^{n}$. Hence, $S^{n}$ is a CW-complex with one 0 -cell and one $n$-cell. For instance, see Figure 6 which shows the CW-complex structure of $S^{2}$. By Lemma 1.37 we have that $H_{n}\left(X^{n}, X^{n-1}\right) \approx \mathbb{Z}$ and $H_{0}\left(X^{0}\right) \approx \mathbb{Z}$. The chain complex is given by

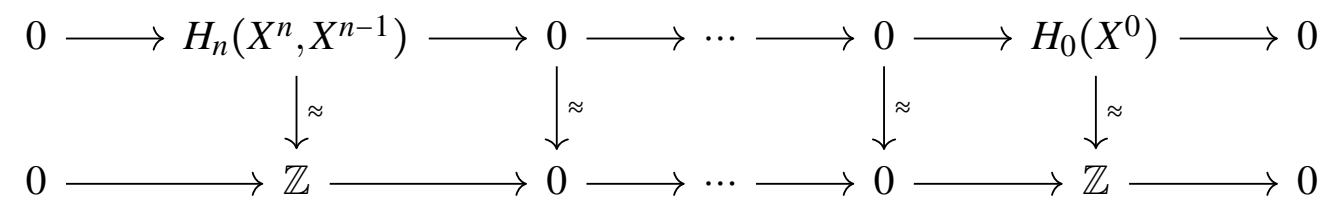




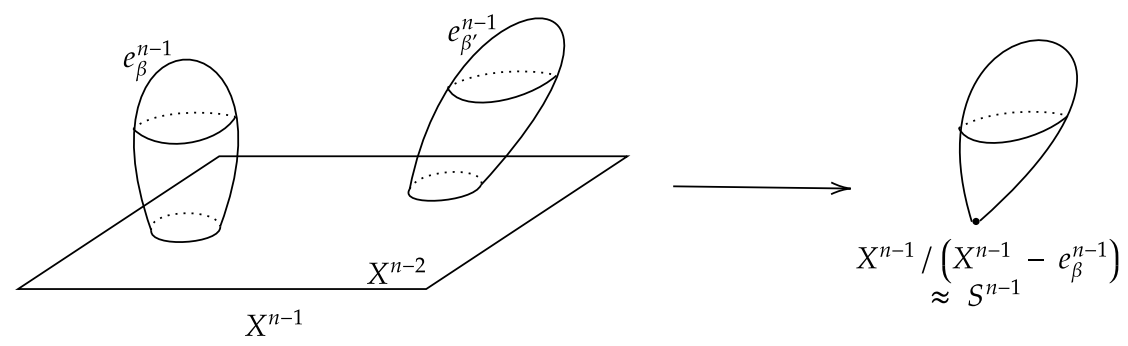

Figure $5-X^{n-1} /\left(X^{n-1}-e_{\beta}^{n-1}\right) \approx S_{\beta}^{n-1}$

Therefore by Theorem 1.38 we have that

$$
H_{k}\left(S^{n}\right)= \begin{cases}\mathbb{Z} & \text { if } k=0, n \\ 0 & \text { otherwise }\end{cases}
$$
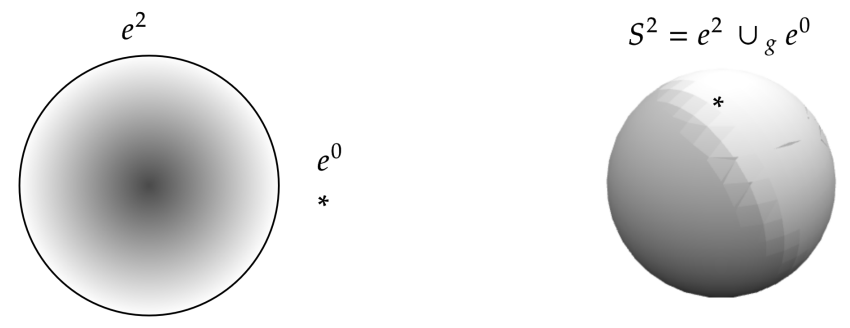

Figure 6 - The CW-complex structure of $S^{2}$.

Example 1.40. The torus $T^{2}$ has the structure of CW-complex. Indeed, it has one 0-cell $e^{0}$, two 1 -cells $e_{1}^{1}, e_{2}^{1}$ and one 2-cell $e^{2}$. The attaching maps of the 1-cells $f_{i}: \partial e_{i}^{1} \rightarrow e^{0}$ is the constant function to a a point $e^{0}$. Then the 1 -skeleton $X^{1}$ is the figure eight, see Figure 7. $e^{2}$ is homoemorphic to $I \times I$, let $L_{1}, L_{2}, L_{3}$ and $L_{4}$ be its the sides oriented as in the Figure 7. Then the attaching map $g: e^{2} \rightarrow X^{1}$ maps $L_{1}$ and $L_{3}$ onto $e_{1}^{1}$, on $L_{1}$ the maps $g$ preserves orientation and on $L_{3}$ the map $g$ reverses orientation; $g$ maps $L_{2}$ and $L_{4}$ onto $e_{2}^{1}$, on $L_{2}$ the map $g$ preserves orientation and on $L_{4}$ the map $g$ reverses orientation. The Figure 8 shows the CW-structure of torus $T^{2}$. Now we will compute the homology groups using Theorem 1.38. By the structure of $\mathrm{CW}$-complex we have the chain complex,

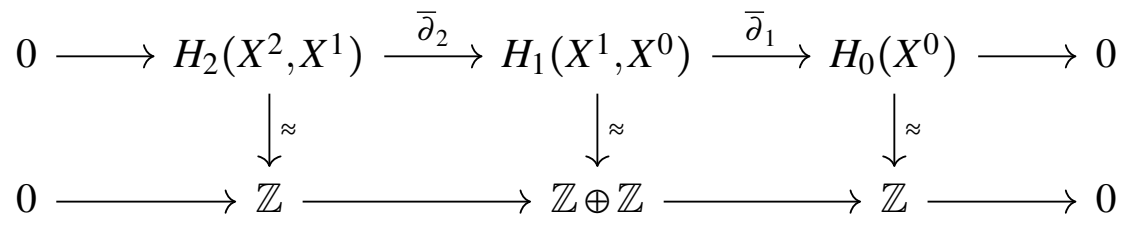



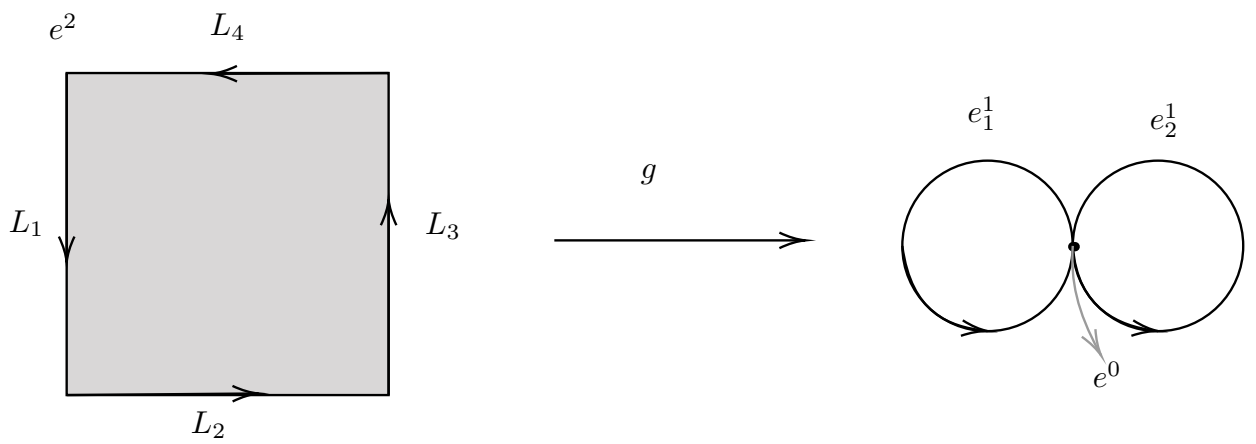

Figure 7 - CW-complex structure of torus $T^{2}$.

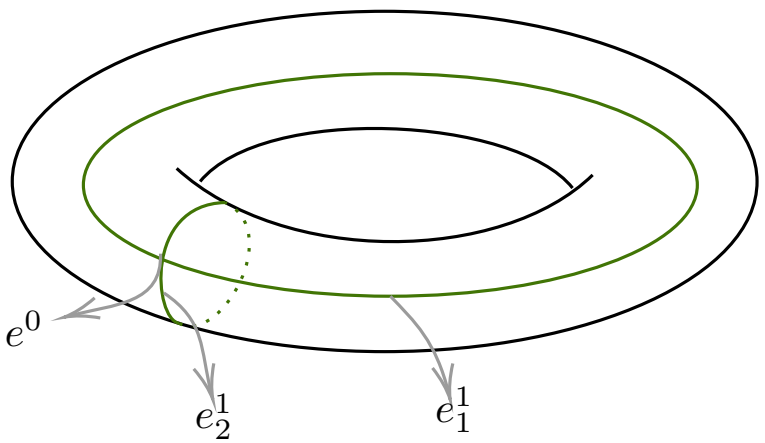

Figure $8-T^{2}$.

Since on $L_{1}$ the map $g$ preserves orientation and on $L_{3}$ the map $g$ reverses orientation, we have that $\bar{\partial}_{2}=0$. Since on $L_{2}$ the map $g$ preserves orientation and on $L_{4}$ the map $g$ reverses orientation, $\bar{\partial}_{1}=0$. Therefore

$$
H_{k}\left(T^{2}\right)=\left\{\begin{array}{ccc}
\mathbb{Z} & \text { if } & k=0,2 \\
\mathbb{Z} \oplus \mathbb{Z} & \text { if } & k=1
\end{array}\right.
$$

We have a useful property of maps between CW-complexes that will help us to prove the main theorem of Section 2.2.

Definition 1.41. Let $f: X \rightarrow Y$ be a continuous map between CW-complexes, we say that $f$ is a cellular map if $f$ maps the $n$-skeleton of $X$ to $n$-skeleton of $Y$, i.e, $f\left(X^{n}\right) \subset f\left(Y^{n}\right)$.

Theorem 1.42 (Cellular approximation Theorem). (HATCHER, 2002, Theorem. 4.8) Every continuous map $f: X \rightarrow Y$ between $\mathrm{CW}$-complexes is homotopic to a cellular map $g: X \rightarrow Y$. Moreover, if $\left.f\right|_{A}$ is already a cellular for a subcomplex $A \subset X$ then the homotopy may be taken to be stationary on $A$, i.e, if $H: X \times I \rightarrow Y$ is the homotopy between $f$ and $g$, then $H(x, t)=f(x)$ for all $x \in A$. 


\subsection{Transversality}

Here we present the classic theorems on transversality which will be widely used in our work. For instance the transversality condition will be used to characterize smooth Morse function on a manifold through a section of the cotangent bundle. See Remark 2.7, item 1. Moreover, this concept will be necessary for studying the stable and unstable manifolds in Chapter 3.

Definition 1.43. Let $M, N$ and $Z$ be a smooth manifolds, and $f: M \rightarrow N, g: Z \rightarrow N$ be a smooth maps. We say that $f$ is transverse to $g$ if whenever $f(x)=g(z)=y$ the following holds

$$
T_{y} N=d f_{x}\left(T_{x} M\right)+d g_{z}\left(T_{z} N\right)
$$

if $Z \subset N$ is an embedded submanifold of $N$ and $g$ is the inclusion map we say that $f$ is transverse to $Z$.

If $M$ and $N$ are smooth submanifold of a smooth manifold $Z$ and $i_{M}: M \hookrightarrow Z$ is the inclusion map we can see the intersection $M \cap N$ as the set $i_{M}^{-1}(N)$ i.e, the map $j: i_{M}^{-1}(N) \hookrightarrow Z$ is an inclusion where $\operatorname{Im}(j)=M \cap N$. One may ask, when the intersection of two manifolds is a manifold? Transversality is a condition that allows us to answer this question.

Theorem 1.44. (LEE, 2013, Corollary. 6.31) Let $f: M \rightarrow N$ be a smooth map, $Z \subset N$ be an embedded submanifold and $g: Z \rightarrow N$ be the inclusion map. If $f$ is transverse to $g$, then $f^{-1}(Z)$ is a submanifold of $M$ whose codimension is equal to the codimension of $Z$ in $N$.

Example 1.45. Let $M$ be a compact smooth manifold and let $f: M \rightarrow \mathbb{R}$ be a smooth function. Then $t_{0} \in \mathbb{R}$ is a regular value if and only if $f$ is transverse to $t_{0}$. Indeed, $f$ is transverse to $t_{0}$ if and only if for all $p \in f^{-1}(p)$ we have that $d f_{p}\left(T_{p} M\right)+T_{t_{0}} t_{0}=T_{t_{0}} \mathbb{R}$ if and only if $d f_{p}\left(T_{p} M\right)=T_{t_{0}} \mathbb{R}$ for all $p \in f^{-1}(p)$ if and only if $t_{0}$ is a regular value of $f$. By previous theorem we have that $f^{-1}(p)$ is a smooth submanifold of $M$ and $\operatorname{dim}\left(f^{-1}(p)\right)=\operatorname{dim} M-1$

Example 1.46. Let $f: M \rightarrow \mathbb{R}$ be a smooth function on a compact Riemannian manifold $(M, g)$. By Corollary 1.16 we know that the gradient vector field $\nabla f$ is complete. Let $t \in \mathbb{R}$ be a regular value of $f$ and $\gamma$ be a flow line of $\nabla f$ such that $\gamma(0)=p \in f^{-1}(t)$. Then $f^{-1}(t)$ is transverse to $\gamma$ at $p$.

Indeed, $\operatorname{dim}\left(f^{-1}(t)\right)=\operatorname{dim} M-1$ and we have that $T_{p}\left(f^{-1}(t)\right)=\operatorname{ker}\left(d f_{p}\right)$. Clearly $\operatorname{dim} \operatorname{Im}(\gamma)=1$. Then $\operatorname{Im}(\gamma)$ and $f^{-1}(t)$ have complementary dimensions, so it is enough to prove that $\gamma^{\prime}(0)=$ $\nabla f(p) \notin \operatorname{ker} d f_{p}$. In fact, $d f_{p}(\nabla f(p))=g(\nabla f(p), \nabla f(p))=\|\nabla f(p)\|_{p} \neq 0$.

In Example 1.21 we have the height function on 2-sphere, the Figure 9 shows the transversality of $\gamma$ with $f^{-1}(p)$ at $p$.

Definition 1.47 ((BANYAGA; HURTUBISE, 2013)). A property of a smooth map $f: M \rightarrow N$ is called locally stable if for all $x \in M$ there is a neighborhood $U \subset M$ such that whenever 


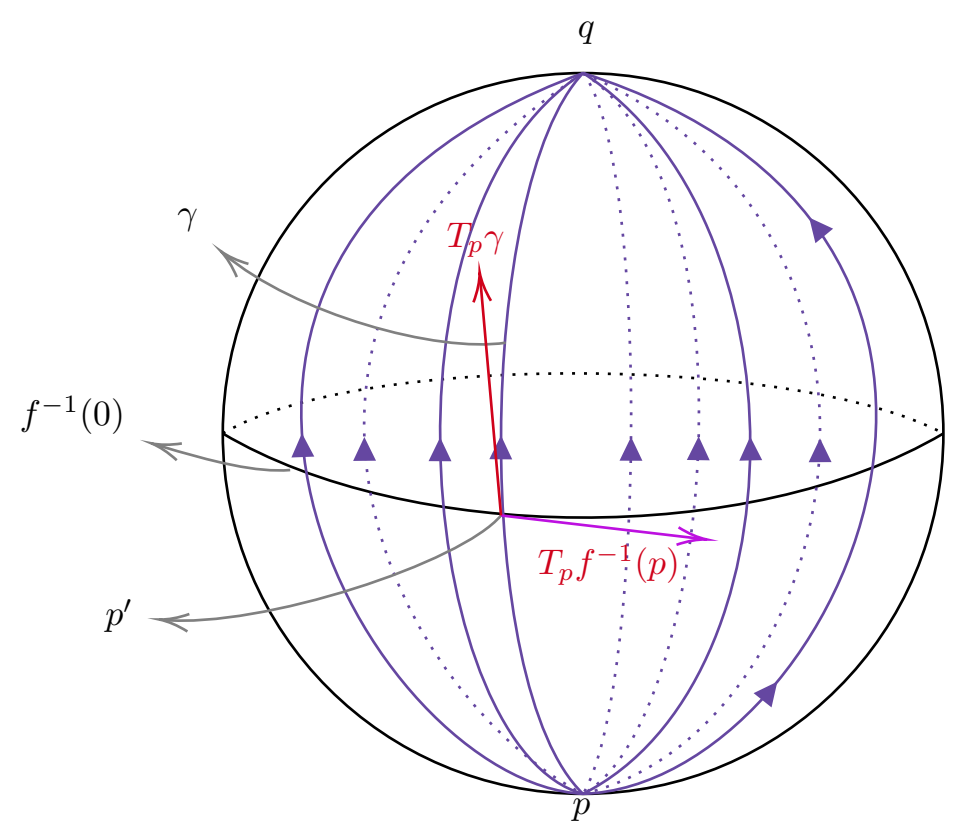

Figure 9 - Transversality of $\gamma$ with $f^{-1}(p)$

$\left.f\right|_{U}: U \rightarrow N$ has the property and $H: U \times I \rightarrow N$ is a smooth homotopy of $\left.f\right|_{U}$ then for some $\varepsilon>0$ each $f_{t}: H(\cdot, t)$ with $t<\varepsilon$ also has the same property. A property is called globally stable if the above condition holds for $U=M$.

Theorem 1.48 (Stability Theorem). (BANYAGA; HURTUBISE, 2013, Theorem. 5.16) In $C^{\infty}(M, N)$ the following properties are locally stables: immersions, submersions, local diffeomorphism, maps transverse to a given properly embedded submanifold $X \subset N$.

The study of stable properties in general is important and useful in several many areas of mathematics like geometry, differential topology, analysis, etc. In particular, the stability of transversality can be used to prove that the set of Morse functions are dense in the set of smooth function on a manifold.

Theorem 1.49. (LEE, 2013, Theorem. 6.36) Let $M$ and $N$ be a smooth manifolds and $Z \subset N$ an embedded submanifold. Every smooth map $f: M \rightarrow N$ is homotopic to a smooth map $g: N \rightarrow M$ that is transverse to $Z$.

\subsection{Intersection number}

In this section we define the oriented intersection number of two embedded submanifolds of complementary dimensions. With this we can define the boundary operator of the MorseSmale-Witten chain complex in Chapter 3. The definitions and results in this section were based in the reference (BANYAGA; HURTUBISE, 2013).

Let $M$ and $Z$ be embedded submanifolds of a manifold $N$ such that $M$ is transverse to $Z$, i,e., the inclusion maps $Z \hookrightarrow N$ and $M \hookrightarrow N$ are transversal. Then we know from Theorem 1.44 
that $M \cap Z$ is a embedded submanifold of $N$ and

$$
\operatorname{dim}(M \cap Z)=\operatorname{dim} M+\operatorname{dim} Z-\operatorname{dim} N
$$

Note that:

- if $\operatorname{dim} M+\operatorname{dim} Z<\operatorname{dim} N$ then $\operatorname{dim}(M \cap Z)<0$. Hence, $M \cap Z=\varnothing$,

- if $\operatorname{dim} M+\operatorname{dim} Z=\operatorname{dim} N$ then $\operatorname{dim}(M \cap Z)=0$. Hence, $M \cap Z$ is a collection of points. Furthermore, if $M$ and $Z$ are closed, and $M$ or $Z$ is compact, then $M \cap N$ is a finite set of points.

Recall that if $f: M \rightarrow N$ is not transversal to $Z$ by Theorem 1.49 there is a map $g$ homotopic to $f$ that is transverse to $Z$.

Now assume that $M, N$ and $Z$ are oriented manifolds where $Z$ is an embedded closed submanifold of $N$ and $M$ is compact. Suppose further that $\operatorname{dim} M+\operatorname{dim} Z=\operatorname{dim} N$ and $f: M \rightarrow N$ a smooth map transverse to $Z$. Then $f^{-1}(Z)$ is a finite collection of points. Given $x \in f^{-1}(Z)$ and $y=f(x)$ by transversality we have that $T_{y} Z+d f_{x}\left(T_{x} M\right)=T_{y} N$.

Let $u_{x}, v_{y}, w_{y}$ be positive oriented bases of $T_{x} M, T_{y} Z$ and $T_{y} N$ respectively. Then $w_{y}^{\prime}=$ $\left(d_{x} f\left(u_{x}\right), v_{y}\right)$ is a new basis of $T_{y} N$.

Definition 1.50. Under previous hypothesis and notation, define the sign of $x$ by,

$$
\operatorname{sign}(x)=\left\{\begin{array}{cll}
1 & \text { if } & w_{y}^{\prime} \text { is postive oriented } \\
-1 & \text { if } & \text { otherwise }
\end{array}\right.
$$

The oriented intersection number, $I(f, Z) \in \mathbb{Z}$, is defined by

$$
I(f, Z)=\sum_{x \in f^{-1}(Z)} \operatorname{sign}(x)
$$

Theorem 1.51. (GUILLEMIN; POLLACK, 1974, p. 108) Let $f, g: M \rightarrow N$ be two homotopic smooth maps which are both transverse to $Z$. Then $I(f, Z)=I(g, Z)$. 
CHAPTER

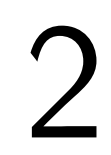

BASIC MORSE THEORY

In this chapter we will introduce the main results of the basic Morse theory. We will present the Morse lemma that gives a local description of a Morse function around its critical points and we do an application proving the Reeb's theorem. Then we will study the homotopy type of a manifold through the Morse function and its critical points. We will also present Morse inequalities, the Morse and Poincare polynomials and their relationship. To finish we present an study of perfect Morse function which makes the Morse inequality be an equality.

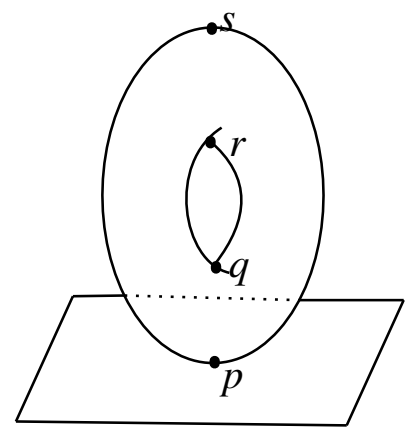

Figure 10 - The torus and points $p, q, r$ and $s$.

The following example motivates the study of Morse theory.

Let $M=T^{2} \subset \mathbb{R}^{3}$ be the torus tangent to the plane $z=0$ and let $f: T^{2} \rightarrow \mathbb{R}$ be the height function. For each real number consider the set $M^{a}:=\{x \in M \mid f(x) \leq a\}$ and the points $p, q, r, s \in M$ as in Figure 10. Let $a_{1}, a_{2}, a_{3}, a_{4}, a_{5} \in \mathbb{R}$ such that

$$
a_{1}<f(p)<a_{2}<f(q)<a_{3}<f(r)<a_{4}<f(s)<a_{5} .
$$

We have the following:

$$
\text { - } M^{a_{1}}=\varnothing
$$


- $M^{a_{2}}$ is homeomorphic to a 2-cell which has the same homotopy type of $M^{a_{1}}$ attatching a 0 -cell.

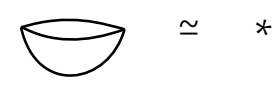

- $M^{a_{3}}$ is homeomorphic to a cylinder which has the same homotopy type of $M^{a_{2}}$ attaching a 1-cell.
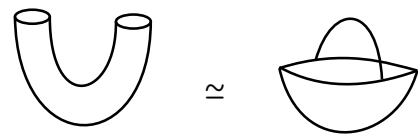

- $M^{a_{4}}$ is homoeomophic to a compact manifold of genus 1 having a circle as boundary, which has the same homotopy type of a $M^{a_{3}}$ attaching a 1-cell.

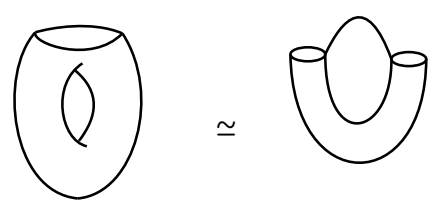

- $M^{a_{5}}$ the torus, which has the same homotopy type of a $M^{a_{4}}$ attaching a 2-cell.

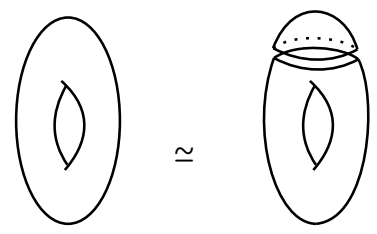

Observe that the homotopy type of $M^{t}$ changes when $t$ goes through the critical values $f(p)$, $f(q), f(r)$ and $f(s)$. Intuitively we see that we can choose local coordinates:

- at $p$ such that $f(x, y)=c+x^{2}+y^{2}$,

- at $q$ and $r$ such that $f(x, y)=c+x^{2}-y^{2}$,

- at $s$ such that $f(x, y)=c-x^{2}-y^{2}$,

and the partial derivatives $\frac{\partial f}{\partial x}$ and $\frac{\partial f}{\partial y}$ are both zero at those points. We will prove that we can find such coordinate system around each critical point of $f$ (See Lemma 2.2). Remark that the number of negative signs in the expression of $f$ at each point is the same as the dimension of the cell attached to $M^{a_{j}}$ to get $M^{a_{j+1}}$ for $j=1,2,3,4$. See Theorem 2.18.

\subsection{Morse Lemma}

The proof of the classical Morse lemma will be based on the following lemma. 
Lemma 2.1. Let $V \subset \mathbb{R}^{n}$ be a convex neighborhood of 0 and $f: V \rightarrow \mathbb{R}$ smooth with $f(0)=0$. Then

$$
f\left(x_{1}, \ldots, x_{n}\right)=\sum_{i=1}^{n} x_{i} g_{i}\left(x_{1}, \ldots, x_{n}\right)
$$

for some suitable smooth functions $g_{i}: V \rightarrow \mathbb{R}$, with $g_{i}(0)=\frac{\partial f}{\partial x_{i}}(0)$.

Proof. We may assume without lost of generality that $V=\mathbb{R}^{n}$. We define $h: \mathbb{R} \rightarrow \mathbb{R}^{n}, t \mapsto$ $\left(t x_{1}, \ldots t x_{n}\right)$, then $f \circ h: \mathbb{R} \rightarrow \mathbb{R}, f \circ h(t)=f\left(t x_{1}, \ldots, t x_{n}\right)$. Therefore we have,

$$
\int_{0}^{1} \frac{d}{d t} f \circ h(t) d t=f\left(x_{1}, \ldots x_{n}\right)
$$

Since,

$$
\begin{aligned}
\frac{d}{d t} f \circ h(t) & =d f(h(t)) d h(t) \\
& =\left(\frac{\partial f}{\partial x_{1}}\left(t x_{1}, \ldots, t x_{n}\right), \ldots, \frac{\partial f}{\partial x_{n}}\left(t x_{1}, \ldots, t x_{n}\right)\right)\left(x_{1}, \ldots, x_{n}\right) \\
& =\sum_{i=1}^{n} x_{i} \frac{\partial f}{\partial x_{i}}\left(t x_{1}, \ldots, t x_{n}\right),
\end{aligned}
$$

we have that

$$
\int_{0}^{1} \frac{d}{d t} f \circ h(t) d t=\sum_{i=1}^{n} x_{i} \int_{0}^{1} \frac{\partial f}{\partial x_{i}}\left(t x_{1}, \ldots, t x_{n}\right) d t .
$$

We define $g_{i}\left(x_{1}, \ldots, x_{n}\right)=\int_{0}^{1} \frac{\partial f}{\partial x_{i}}\left(t x_{1}, \ldots, t x_{n}\right) d t$. Then,

$$
f\left(x_{1}, \ldots, x_{n}\right)=\sum_{i=1}^{n} x_{i} g_{i}\left(x_{1}, \ldots, x_{n}\right)
$$

The following theorem is the main result of this section which state that given a nondegenerate critical point of a smooth function one can find a nice local description of the function around that critical point.

Lemma 2.2 (Morse Lemma). Let $M$ be a smooth manifold and let $f: M \rightarrow \mathbb{R}$ be a smooth function. Suppose that $p$ is a non-degenerated critical point of $f$, then there is a local coordinate system $\left(y_{1}, \ldots, y_{m}\right)$ in a neighborhood $U$ of $p$ with $y_{i}(p)=0$ for $i=1, \ldots, m$, such that

$$
f\left(\left(y_{1}, \ldots, y_{m}\right)\right)=f(0)-y_{1}^{2}-\cdots-y_{\lambda}^{2}+\cdots+y_{m}^{2}
$$

for all $q \in U$, where $\lambda$ is the index of $f$ at $p$.

Proof. Suppose that there exist the local coordinate system $\left(y_{1}, \ldots, y_{m}\right)$ that satisfies (2.1). Then, $\lambda$ is the index of $f$ at $p$, i.e., $\lambda$ is the index of the bilinear functional $f_{\star *}$ on $T_{p} M$ by 
Subsection 1.1.2. Indeed, we know that matrix of $f_{* *}$ at $p$ in the base $\left(\left.\frac{\partial}{\partial y_{1}}\right|_{p}, \ldots,\left.\frac{\partial}{\partial y_{m}}\right|_{p}\right)$ is $H_{p}(f)=\left(\frac{\partial^{2} f}{\partial y_{i} \partial y_{j}}(p)\right)$, where

$$
\frac{\partial^{2} f}{\partial y_{i} \partial y_{j}}(p)=\left\{\begin{array}{rll}
-2 & \text { if } & i=j \leq \lambda \\
2 & \text { if } & i=j>\lambda \\
0 & \text { if } & i \neq j
\end{array}\right.
$$

Hence, $H_{p}(f)$ is a diagonal matrix:

$$
H_{f}(p)=\left(\begin{array}{llllll}
-2 & & & & & \\
& \ddots & & & & \\
& & -2 & & & \\
& & & 2 & & \\
& & & & \ddots & \\
& & & & & 2
\end{array}\right) .
$$

Therefore the Morse index of $f$ at $p$ is equal to $\lambda$.

Now we must show that such coordinate system $\left(y_{1}, \ldots, y_{m}\right)$ exists. By translation we can assume that $y_{i}(p)=0$ for $i=1, \ldots, m$ and $f(p)=f(0)=0$. Let $\left(x_{1}, \ldots, x_{m}\right)$ be a local coordinate system in a convex neighborhood $V$ of 0 , then by Lemma 2.1 we have that

$$
f\left(x_{1}, \ldots, x_{m}\right)=\sum_{i=1}^{m} x_{i} g_{i}\left(x_{1}, \ldots, x_{m}\right)
$$

for some suitable smooth functions $g_{i}: V \rightarrow \mathbb{R}$, with $g_{i}(0)=\frac{\partial f}{\partial x_{i}}(0)$. Note that $g_{i}$ also satisfies the conditions of the Lemma 2.1, hence there are a suitable smooth functions $h_{i j}$ such that

$$
g_{i}\left(x_{1}, \ldots, x_{m}\right)=\sum_{j=1}^{m} x_{j} h_{i j}\left(x_{1}, \ldots, x_{m}\right) .
$$

So, we have that

$$
\begin{aligned}
f\left(x_{1}, \ldots, x_{m}\right) & =\sum_{i, j=1}^{m} x_{i} x_{j} h_{i j}\left(x_{1}, \ldots, x_{m}\right) \\
& =\sum_{i=1}^{m} x_{i}^{2} h_{i i}\left(x_{1}, \ldots, x_{m}\right)+\sum_{i<j} x_{i} x_{j}\left(h_{i j}+h_{j i}\right)\left(x_{1}, \ldots, x_{m}\right) .
\end{aligned}
$$

Define $h_{i j}^{\prime}=\frac{1}{2}\left(h_{i j}+h_{j i}\right)$, then $h_{i j}^{\prime}=h_{j i}^{\prime}$ and

$$
f\left(x_{1}, \ldots, x_{m}\right)=\sum_{i, j=1}^{m} x_{i} x_{j} h_{i j}^{\prime}\left(x_{1}, \ldots, x_{m}\right) .
$$

Note that the matrix $\left(h_{i j}^{\prime}(0)\right)_{i, j=1 \ldots, m}=2 H_{0}(f)$. Since 0 is non-degenerate critical point, the matrix $\left(h_{i, j}^{\prime}(0)\right)$ is non-singular. Now we will show by recursion that there is a change of coordinate system $\left(y_{1}, \ldots, y_{m}\right)$ such that $f\left(y_{1}, \ldots, y_{m}\right)=f(p)-y_{1}^{2}-\cdots-y_{\lambda}^{2}+\cdots+y_{m}^{2}$ in a perhaps 
smaller neighborhood $V$ of 0 .

Suppose that there exists a coordinate system $\left(u_{1}, \ldots, u_{m}\right)$ in a neighborhood $U_{1}^{\prime}$ such that

$$
f\left(u_{1}, \ldots, u_{m}\right)= \pm u_{1}^{2} \pm \cdots \pm u_{r-1}^{2}+\sum_{i, j \geq r} u_{i} u_{j} h_{i, j}^{\prime \prime}\left(u_{1}, \ldots, u_{m}\right) .
$$

where the matrix $\left(h_{i, j}^{\prime \prime}(0)\right)_{i, j=r, \ldots, m}$ is non-singular. Hence, we can suppose that $h_{r r}^{\prime \prime}(0) \neq 0$. In order to reduce notation, denote $\left(u_{1}, \ldots, u_{m}\right)$ by $u$. Then we have that

$$
f(u)= \pm u_{1}^{2} \pm \cdots \pm u_{r-1}^{2}+\underbrace{u_{r r}^{2} h_{r r}^{\prime \prime}(u)+\sum_{j=r+1}^{m} u_{r} u_{j} h_{r j}^{\prime \prime}(u)+\sum_{i=r+1}^{m} u_{i} u_{r} h_{i r}^{\prime \prime}(u)}_{\mathbf{A}}+\sum_{i, j=r+2}^{m} u_{i} u_{j} h_{i j}^{\prime \prime}(u),
$$

The part $\mathbf{A}$ is equal to

$$
\begin{aligned}
& u_{r}^{2} h_{r r}^{\prime \prime}(u)+2 u_{r} \sum_{j=r+1}^{m} u_{j} h_{r j}^{\prime \prime}(u)=h_{r r}^{\prime \prime}(u)\left(u_{r}^{2}+2 u_{r} \sum_{j=r+1}^{m} u_{j} \frac{h_{r j}^{\prime \prime}(u)}{h_{r r}^{\prime \prime}(u)}\right) \\
& =h_{r r}^{\prime \prime}(u)\left(u_{r}^{2}+2 u_{r} \sum_{j=r+1}^{m} u_{j} \frac{h_{r j}^{\prime \prime}(u)}{h_{r r}^{\prime \prime}(u)}+\left(\sum_{j=r+1}^{m} u_{j} \frac{h_{r j}^{\prime \prime}(u)}{h_{r r}^{\prime \prime}(u)}\right)^{2}\right)-h_{r r}^{\prime \prime}(u)\left(\sum_{j=r+1}^{m} u_{j} \frac{h_{r j}^{\prime \prime}(u)}{h_{r r}^{\prime \prime}(u)}\right)^{2} \\
& =h_{r r}^{\prime \prime}(u)\left(u_{r}+\sum_{j=r+1}^{m} u_{j} \frac{h_{r j}^{\prime \prime}(u)}{h_{r r}^{\prime \prime}(u)}\right)^{2}-h_{r r}^{\prime \prime}(u)\left(\sum_{j=r+1}^{m} u_{j} \frac{h_{r j}^{\prime \prime}(u)}{h_{r r}^{\prime \prime}(u)}\right)^{2} .
\end{aligned}
$$

Consider map $\left(u_{1}, \ldots, u_{r}, \ldots, u_{m}\right) \mapsto\left(v_{1}, \ldots, v_{r}, \ldots, v_{m}\right)$ where $v_{i}=u_{i}$ for $i \neq r$ and

$$
v_{r}=\left|h_{r r}^{\prime \prime}(u)\right|^{1 / 2}\left(u_{r r}+\sum_{j=r+1}^{m} u_{j} \frac{h_{r j}^{\prime \prime}(u)}{h_{r r}^{\prime \prime}(u)}\right) .
$$

Its Jacobian at origin is

$$
\operatorname{det}\left(\left(\begin{array}{cccccc}
1 & 0 & \cdots & 0 & \cdots & 0 \\
0 & 1 & \cdots & 0 & \cdots & 0 \\
\vdots & \vdots & \ddots & \vdots & \ddots & \vdots \\
\frac{\partial v_{r}}{\partial u_{1}}(0) & \frac{\partial v_{r}}{\partial u_{1}}(0) & \cdots & \frac{\partial v_{r}}{\partial u_{r}}(0) & \cdots & \frac{\partial v_{r}}{\partial u_{m}}(0) \\
\vdots & \vdots & \ddots & \vdots & \ddots & \vdots \\
0 & 0 & \cdots & 0 & \cdots & 1
\end{array}\right)\right)=\frac{\partial v_{r}}{\partial u_{r}}(0)
$$

where

$$
\frac{\partial v_{r}}{\partial u_{r}}(0)=\frac{\partial}{\partial u_{r}}\left(\left|h_{r r}^{\prime \prime}(u)\right|^{1 / 2}\left(u_{r r}+\sum_{j=r+1}^{m} u_{j} \frac{h_{r j}^{\prime \prime}(u)}{h_{r r}^{\prime \prime}(u)}\right)\right)(0)=\left|h_{r r}^{\prime \prime}(0)\right|^{1 / 2} \neq 0 .
$$

Then, by the Inverse Function Theorem, there is a neighborhood of $V_{2}$ of 0 where the map $\left(u_{1}, \ldots, u_{m}\right) \mapsto\left(v_{1}, \ldots, v_{m}\right)$ is a diffeomorphism. Therefore, in the local coordinate system $\left(v_{1}, \ldots, v_{m}\right)$ we have that

$$
f\left(v_{1}, \ldots, v_{m}\right)=\sum_{i=1}^{r} \pm v_{i}^{2}+\sum_{i, j=r+1}^{m} v_{i} v_{j} h_{i j}^{\prime \prime \prime}\left(v_{1}, \ldots, v_{m}\right) .
$$


In the step $r=m+1$ we obtain a coordinate system $\left(v_{1}, \ldots, v_{m}\right)$ such that

$$
f\left(v_{1}, \ldots, v_{m}\right)= \pm v_{1}^{2} \pm v_{2}^{2} \pm \cdots \pm v_{m-1}^{2} \pm v_{m}^{2} h(v)
$$

for some function $h$ with $h(0) \neq 0$. By the same argument above the map $\left(v_{1}, \ldots, v_{m}\right) \mapsto$ $\left(y_{1}, \ldots, y_{m}\right)$ where $y_{i}=v_{i}$ for $i \neq m$ and $y_{i}= \pm u_{m}\left|h\left(u_{1}, \ldots, u_{m}\right)\right|^{1 / 2}$ is a diffoomorphism on a neightborhood $V$ of 0 . Therefore, in the coordinate system $\left(y_{1}, \ldots, y_{m}\right)$ we have that

$$
f\left(y_{1}, \ldots, y_{m}\right)= \pm y_{1}^{2} \pm \cdots \pm y_{m}^{2} .
$$

Corollary 2.3. Non-degenerate critical points are isolated.

Proof. Let $f: \mathbb{R}^{n} \rightarrow \mathbb{R}$ be a smooth function with a non-degenerate critical point at $p \in \mathbb{R}^{n}$. By Morse Lemma there is a local coordinate system $\phi=\left(y_{1}, \ldots, y_{n}\right)$ in a neighborhood $U$ of $p$ with $y_{i}(p)=0$ for all $i=1, \ldots, n$ and such that

$$
f\left(\left(y_{1}, \ldots, y_{n}\right)\right)=f(p)-y_{1}^{2}-\cdots-y_{\lambda}^{2}+\cdots+y_{n}^{2}, \text { for all }\left(y_{1}, \ldots, y_{n}\right) \in U
$$

where $\lambda$ is the index of $f$ at $p$. Since $\frac{\partial f}{\partial y_{i}}\left(\left(y_{1}, \ldots, y_{n}\right)\right)=2 y_{i}$ for $i=1, \ldots, n, \frac{\partial f}{\partial y_{i}}\left(\left(y_{1}, \ldots, y_{n}\right)\right)=0$ if and only if $\left(y_{1}, \ldots, y_{n}\right)=0$. Hence, $(0, \ldots, 0) \in \mathbb{R}^{n}$ is the only critical point of $f \circ \phi^{-1}$ on $\phi(U)$. Then $p$ is a isolated critical point of $f$.

Remark that the converse statement of previous corollary is not true, that is, there exists isolated critical points such that it is not non-degenerate as shown in the following example.

Example 2.4. Consider the function $f: \mathbb{R} \rightarrow \mathbb{R}, x \mapsto x^{3}$. Then the differential of $f$ at a point $p$ is $d_{p} f: T_{p} \mathbb{R} \rightarrow T_{p^{3}} \mathbb{R},\left.\left.\frac{\partial}{\partial x}\right|_{p} \mapsto 3 p^{2} \frac{\partial}{\partial x}\right|_{p^{3}}$. Hence, 0 is the unique critical point of $f$. Since the Hessian of $f$ at 0 is $H_{f}(0)=(0), 0$ is a degenerate critical point of $f$.

The Morse Lemma give us a description of non-degenerate critical points which are local minimum and local maximum points.

Example 2.5. Let $f: M \rightarrow \mathbb{R}$ be a smooth function, Suppose that $f$ has a non-degenerate maximal and minimal point $p_{+}$and $p_{-}$respectively. Then, there is a charts $(U, \varphi)$ and $(V, \phi)$ of $p_{+}$and $p_{-}$respectively such that

$$
\begin{aligned}
& f \circ \varphi^{-1}\left(x_{1}, \ldots, x_{m}\right)=f\left(p_{+}\right)-x_{1}^{2}-\cdots-x_{m}^{2}, \text { for }\left(x_{1}, \ldots, x_{m}\right) \in \varphi(U) \\
& g \circ \phi^{-1}\left(x_{1}, \ldots, x_{m}\right)=f\left(p_{-}\right)+x_{1}^{2}+\cdots+x_{m}^{2}, \text { for }\left(x_{1}, \ldots, x_{m}\right) \in \phi(V) .
\end{aligned}
$$

Therefore, the index of a non-degenerate maximal critical point is equal to the dimension of the manifold, and the index of a non-degenerate minimal critical point is 0 .

Definition 2.6 (Morse function). A smooth function $f: M \rightarrow \mathbb{R}$ on a manifold $M$ is called Morse function if its critical points are non-degenerate. 
Remark 2.7. It is possible to prove that:

1. Given a smooth function $f: M \rightarrow \mathbb{R}$ and $p$ a critical point of $f$, then $p$ is a non-degenerate critical point if and only if the smooth map $d f: M \rightarrow T^{*} M, q \mapsto T_{q} M$, is transverse to zero section of the projection map $\pi: T^{*} M \rightarrow M$ at $p$ where $T^{*} M$ is the cotangent bundle of $M$. Fore more details see (BANYAGA; HURTUBISE, 2013, Lemma. 5.23)

2. If $\Omega(M)$ is the set of Morse function on $M$, then with Whitney topology $\Omega(M)$ is dense on $C^{\infty}(M)$. See (BANYAGA; HURTUBISE, 2013, Theorem. 5.27)

Example 2.8. Consider the Example 1.22. The function $f: T^{2} \rightarrow \mathbb{R}$ where $f(x, y)=\cos (2 \pi x)+$ $\cos (2 \pi y)$ is a Morse function on the torus $T^{2}$. The critical points are:

- the point $p=(1 / 2,1 / 2)$ of index 0 which is a minimum,

- two critical point of index 1 , the points $q=(1 / 2,0)$ and $r=(0,1 / 2)$, and

- the point $(0,0)$ of index 2 which is a maximum.

Indeed, we have that

$$
H(f)(x, y)=\left(\begin{array}{cc}
-4 \pi^{2} \cos (2 \pi) & 0 \\
0 & -4 \pi^{2} \cos (2 \pi)
\end{array}\right) .
$$

Therefore, the calculation of the indices are,

$$
\begin{aligned}
H(f)(1 / 2,1 / 2) & =\left(\begin{array}{cc}
4 \pi^{2} & 0 \\
0 & 4 \pi^{2}
\end{array}\right), \\
H(f)(1 / 2,0) & =\left(\begin{array}{cc}
4 \pi^{2} & 0 \\
0 & -4 \pi^{2}
\end{array}\right), \\
H(f)(0,1 / 2) & =\left(\begin{array}{cc}
-4 \pi^{2} & 0 \\
0 & 4 \pi^{2}
\end{array}\right), \\
H(f)(0,0) & =\left(\begin{array}{cc}
-4 \pi^{2} & 0 \\
0 & -4 \pi^{2}
\end{array}\right) .
\end{aligned}
$$

Example 2.9. Consider the complex projective space. Let

$$
S^{2 n+1}=\left\{z=\left.\left(z_{0}, \ldots, z_{n}\right) \in \mathbb{C}^{n+1}|| z\left|:=\sum_{k=0}^{n}\right| z_{k}\right|^{2}=1\right\}
$$

be the $(2 n+1)$-sphere in $\mathbb{C}^{n+1}$. We have the following natural action of the 1 -sphere $S^{1}=\{z \in \mathbb{C} \mid$ $|z|=1\}$ on $S^{2 n+1}$,

$$
\begin{aligned}
S^{1} \times S^{2 n+1} & \longrightarrow S^{2 n+1} \\
\left(s,\left(z_{0}, \ldots, z_{n}\right)\right) & \longmapsto\left(s z_{0}, \ldots, s z_{n}\right)
\end{aligned}
$$


then we obtain the complex projective space

$$
\mathbb{C} P^{n}=S^{2 n+1} / S^{1},
$$

i.e., if we denote the class of $\left(z_{0}, \ldots, z_{n}\right)$ in $S^{2 n+1} / S^{1}$ by $\left[z_{0}: \ldots: z_{n}\right]$, then $\left[z_{0}: \ldots: z_{n}\right]=\left[z_{0}^{\prime}: \ldots: z_{n}^{\prime}\right]$ if and only if there exists $s \in S^{1}$ such that $\left(z_{0}, \ldots, z_{n}\right)=\left(s z_{0}^{\prime}, \ldots, s z_{n}^{\prime}\right)$. The topology of $\mathbb{C} P^{n}$ is given by the quotient map $S^{2 n+1} \rightarrow \mathbb{C} P^{n}$. Define the function

$$
\begin{aligned}
f: S^{2 n+1} & \longrightarrow \mathbb{R} \\
\left(z_{0}, \ldots, z_{n}\right) & \longmapsto \sum_{k=1}^{n} k\left|z_{k}\right|^{2} .
\end{aligned}
$$

The function $f$ defines a function on the quotient $S^{2 n+1} / S^{1}$,

$$
\begin{aligned}
\bar{f}: S^{2 n+1} / S^{1} & \longrightarrow \mathbb{R} \\
z & \longmapsto f(z) .
\end{aligned}
$$

$\bar{f}$ is well defined because $f(s z)=f(z)$ for all $z \in S^{2 n+1}$ and $s \in S^{1}$.

Now, we will prove that $\bar{f}$ is a Morse function on $\mathbb{C} P^{n}$.

The space $\mathbb{C} P^{n}$ is covered by the $n+1$ open sets

$$
U_{j}=\left\{\left[z_{0}: \cdots: z_{n}\right] \in \mathbb{C} P^{n} \mid z_{j} \neq 0\right\} .
$$

Since in $U_{j}$ we have that $z_{j} \neq 0$ and $\frac{z_{j}}{\left|z_{j}\right|} \in S^{1}$,

$$
\left[z_{0}, \ldots, z_{n}\right]=\left[\frac{z_{j}}{\left|z_{j}\right|} z_{0}, \ldots, \frac{z_{j}}{\left|z_{j}\right|} z_{n}\right] .
$$

Define the charts

$$
\begin{aligned}
\phi: U_{j} & \longrightarrow \mathbb{R}^{2 n} \\
{\left[z_{0}: \cdots: z_{n}\right] } & \longmapsto\left(x_{0}, \ldots, \hat{x}_{j}, \ldots, x_{n}, y_{0}, \ldots, \hat{y}_{j}, \ldots, y_{n}\right)
\end{aligned}
$$

where $\frac{z_{j}}{\left|z_{j}\right|} z_{k}=x_{k}+i y_{k}$ with $k \neq j$. It is clear that $\left|z_{k}\right|^{2}=x_{k}^{2}+y_{k}^{2}$ for $k \neq j$. Since $\left(z_{0}, \ldots, z_{n}\right) \in S^{2 n+1}$,

$$
\left|z_{j}\right|^{2}=1-\sum_{k \neq j}\left|z_{k}\right|^{2}=1-\sum_{k \neq j} x_{k}^{2}+y_{k}^{2}
$$

Then, in $U_{j}$ we have

$$
\begin{aligned}
f\left(z_{0}, \ldots, z_{n}\right) & =\sum_{k=1}^{m} k\left|z_{k}\right|^{2} \\
& =j\left|z_{j}\right|^{2}+\sum_{k \neq j} k\left|z_{k}\right|^{2} \\
& =j\left(1-\sum_{k \neq j}\left|z_{k}\right|^{2}\right)+\sum_{k \neq j} k\left|z_{k}\right|^{2} \\
\bar{f} \circ \phi^{-1}(\phi(z)) & =j+\sum_{k \neq j}(k-j)\left(x_{k}^{2}+y_{k}^{2}\right) .
\end{aligned}
$$


Hence,

$d \bar{f}(z)=\left(2(0-j) x_{0}, \ldots,-2 x_{j-1}, 2 x_{j+1}, \ldots, 2(n-j) x_{n}, 2(0-j) y_{0}, \ldots,-2 y_{j-1}, 2 y_{j+1}, \ldots, 2(n-j) y_{n}\right)$, another way to write that is as follows: if $\left\{e_{i}\right\}_{i=1 \ldots, 2 n}$ is the canonical base of $\mathbb{R}^{2 n}$, then

$$
d \bar{f}(z)=2 \sum_{k \neq j}(k-j)\left(x_{k} e_{k}+y_{k} e_{k+n}\right) .
$$

Then, the point

$$
p_{j}=\phi(0)=\left[0, \ldots, 0, z_{j}, 0, \ldots, 0\right]=[0, \ldots, 0,1,0, \ldots, 0]
$$

is the unique critical point of $f$ on $U_{j}$.

Hence, the function $\bar{f}$ has $n+1$ critical points $p_{j}=\left[e_{j}\right]$ for $j=0, \ldots, n$ and its Hessian is

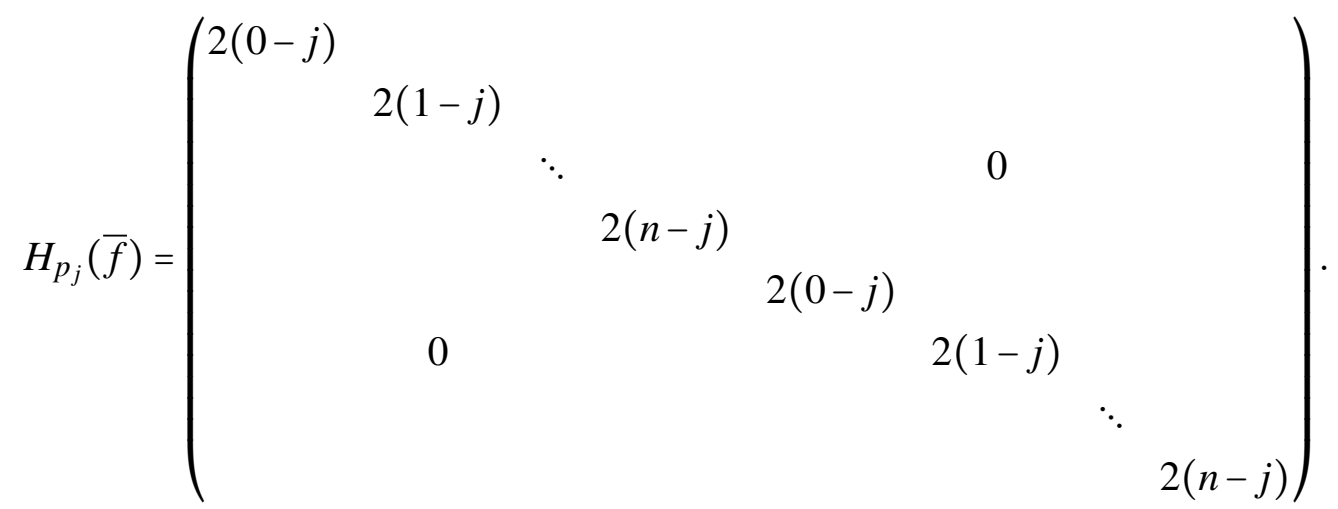

Since there are $2 j$ negative entries on the diagonal, we conclude that the index of $\bar{f}$ at $p_{j}$ is $\lambda_{p_{j}}=2 j$.

Let us remind that given a Morse function $f$ on a manifold $M$ and its gradient flow $\phi$, by Morse lemma we have that a critical point $p$ of $f$ is a hyperbolic fixed point of $\phi_{t}: M \rightarrow M$. This property will be useful because it allows us to apply $\lambda$-lemma and show properties of stable and unstable manifolds of this flow. See Chapter 3.

Definition 2.10. A fixed point $p \in M$ of a smooth diffeomorphism $\varphi: M \rightarrow M$ is called hyperbolic fixed point if none of the eigenvalues of $d_{p} \varphi$ have length 1 .

Remark 2.11. Let $p$ be a fixed point of a diffeomorphism $\varphi: M \rightarrow M$ and $\psi=\left(x_{1} \ldots, x_{m}\right)$ be a local coordinate system in a neighborhood of $p$. Then, $q=\psi(p)$ is an hyperbolic fixed point of $\Phi=\psi \circ \varphi \circ \psi^{-1}$ if and only if $p$ is an hyperbolic fixed point of $\varphi$. Indeed, we have that $d_{q} \Phi=d_{q}\left(\psi \circ \varphi \circ \psi^{-1}\right)=\left(d_{p} \psi\right)\left(d_{p} \varphi\right)\left(d_{q} \psi^{-1}\right)=\left(d_{p} \psi\right)\left(d_{p} \varphi\right)\left(d_{q} \psi\right)^{-1}$, then the linear maps $d_{p} \varphi$ and $d_{q} \Phi$ are similar. Therefore, follows from linear algebra that $d_{q} \Phi$ and $d_{p} \varphi$ must have the same eigenvalues.

Proposition 2.12. Let $f: M \rightarrow \mathbb{R}$ be a Morse function on a compact $m$-dimensional smooth manifold $M$ and $\phi$ be the global flow generated by the vector field $-\nabla f$. Then, the critical points of $f$ are hyperbolic fixed points of $\phi_{t}$ for each $t \neq 0$. 
Proof. By Morse lemma there is a local chart $\left(U,\left(x_{1}, \ldots, x_{m}\right)\right)$ such that $f=f(p)-x_{1}^{2}-\cdots-x_{\lambda}^{2}+$ $x_{\lambda+1}^{2}+\cdots+x_{m}^{2}$, thus

$$
H_{p}(f)=\left(\begin{array}{cccccc}
-2 & & & & & \\
& \ddots & & & 0 & \\
& & -2 & & & \\
& & & 2 & & \\
& 0 & & & \ddots & \\
& & & & & 2
\end{array}\right)
$$

and by Lemma 1.24 we have $d_{x} \phi_{t}(x)=e^{-H_{p}(f) t}$. Hence

$$
d_{x} \phi_{t}(x)=\left(\begin{array}{cccccc}
e^{2 t} & & & & & \\
& \ddots & & & 0 & \\
& & e^{2 t} & & & \\
& & e^{-2 t} & & \\
& 0 & & & \ddots & \\
& & & & & e^{-2 t}
\end{array}\right)
$$

since for $t \neq 0$ we have that $e^{2 t} \neq 1$ and $e^{-2 t} \neq 1$. Therefore, we conclude that $d_{x} \phi_{t}$ has no eigenvalues of length 1 for $t \neq 0$.

\subsection{Morse function and homeomorphism}

In this section we will study the sets $M^{t}=f^{-1}((-\infty, t])$ to see how the homotopy type of $M^{t}$ changes when $t$ goes through critical values of a Morse function $f: M \rightarrow \mathbb{R}$.

Theorem 2.13. Let $f: M \rightarrow \mathbb{R}$ be a smooth function on a Riemmanian manifold $M$. Let $a \leq b$ and suppose that the set $f^{-1}([a, b])$ is compact and contains no critical points of $f$. Then:

1). $M^{a}$ is diffeomorphic to $M^{b}$.

2). $M^{a}$ is a deformation retract of $M^{b}$ such that the inclusion map $i: M^{a} \rightarrow M^{b}$ is a homotopy equivalence.

3). Moreover, there is a diffeomorphism $F$ such that the following diagram is commutative,

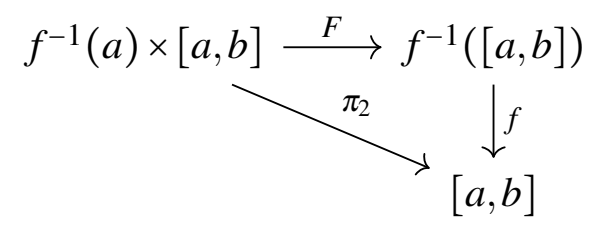

where $\pi_{2}$ is the canonical projection. 
Proof of 1). For the first part let $\langle$,$\rangle be a Riemannian metric on M$. By definition of the gradient vector field (see Definition 1.17) we have that

$$
\langle X, \nabla f\rangle=X(f)
$$

for any smooth vector field $X$ on $M$. Define the following smooth map $\rho: M \rightarrow \mathbb{R}$ by

$$
\rho(p)=\left\{\begin{array}{clc}
\frac{1}{\left\langle\nabla f_{p}, \nabla f_{p}\right\rangle} & \text { if } & p \in f^{-1}([a, b]) \\
0 & \text { if } & p \notin K
\end{array}\right.
$$

where $K$ is a compact set such that $f^{-1}([a, b]) \subset K$. The map $\rho$ is well defined and smooth due to partitions of unity.

Since the vector field $X=\rho \nabla f$ satisfies the hypotheses of the Corollary 1.16, $X$ generates a unique global flow $\theta: \mathbb{R} \times M \rightarrow M$ of $M$. If $q \in f^{-1}([a, b])$, then for all $t_{0}$ such that $q^{\prime}=\theta^{q}\left(t_{0}\right) \in f^{-1}([a, b])$ we have

$$
\frac{d\left(f \circ \theta^{q}\right)}{d t}\left(t_{0}\right)=X_{q^{\prime}}(f)=\left\langle X_{q^{\prime}}, \nabla f_{q^{\prime}}\right\rangle=\rho\left(q^{\prime}\right)\left\langle\nabla f_{q^{\prime}}, \nabla f_{q^{\prime}}\right\rangle=1 .
$$

Claim: Then $\left.\theta_{b-a}\right|_{M^{a}}: M^{a} \rightarrow M^{b}$ is a diffeomorphism from $M^{a}$ to $M^{b}$.

Indeed, if $p \in M^{a}$ and $p \notin K$ we have that $\theta_{b-a}(p)=p \in M^{a} \subset M^{b}$. If $p \in K$ define $t_{0}=\min \{t \mid$ $\left.f \circ \theta_{t}(p)=a\right\}$. If $t_{0}>b-a$ then $f \circ \theta_{b-a}(p)<a$ and $\theta_{b-a}(p) \in M^{a} \subset M^{b}$. If $t_{0} \leq b-a$ let $t_{1}=b-a-t_{0}$, then

$$
f \circ \theta_{b-a}(p)=f \circ \theta_{t_{1}+t_{0}}(p)=f \circ \theta_{t_{1}}(\underbrace{\theta_{t_{0}}(p)}_{\in f^{-1}(a)})=t_{1}+f\left(\theta_{t_{0}}(p)\right)=t_{1}+a \leq b,
$$

and $\theta_{b-a}(p) \in M^{b}$. Hence, $\left.\theta_{b-a}\right|_{M^{a}}$ is well defined. Since $\theta$ is a flow, by the same argument we can prove that the map $\left.\theta_{a-b}\right|_{M^{b}}$ is the inverse of $\left.\theta_{b-a}\right|_{M^{a}}$. This prove the claim.

Proof of 2). Now, we will to prove that $M^{a}$ is a deformation retract of $M^{b}$. Let $r: I \times M^{b} \rightarrow M^{b}$ be defined by

$$
r(t, p)=\left\{\begin{array}{ccc}
p & \text { if } & f(p) \leq a \\
\theta_{t(a-f(p))}(p) & \text { if } & a \leq f(p) \leq b .
\end{array}\right.
$$

The function $r$ is continuous because for $a=f(p)$ we have that $\theta_{t(a-f(p))}(p)=\theta_{0}(p)=p$. Moreover, if $p \in M^{b}-M^{a}$ then $p \in f^{-1}((a, b])$ and

$$
r(1, p)=\theta_{1(a-f(p))}(p)=a-f(p)+f(p)=a .
$$

Therefore, $r\left(1, M^{b}\right)=M^{a}$ and $M^{a}$ is a deformation retract of $M^{b}$ through $r$ and by Remark 1.30 the inclusion map $i: M^{a} \hookrightarrow M^{b}$ is a homotopy equivalence.

Proof of 3). Define the map $F$ as follows

$$
\begin{aligned}
F: f^{-1}(a) \times[a, b] & \longrightarrow f^{-1}([a, b]) \\
(p, t) & \longmapsto \theta_{t-a}(p),
\end{aligned}
$$


by the computation above it is well defined.

Defina $G: f^{-1}([a, b]) \rightarrow f^{-1}(a) \times[a, b]$ by $G(q)=\left(\theta_{a-f(q)}(q), f(q)\right)$. By (2.4) for $q \in f^{-1}([a, b])$ we have that $f\left(\theta_{a-f(q)}(q)\right)=f(q)+(a-f(q))=a$ and hence $G$ is well defined and smooth. Moreover, the map $G$ is the inverse function of $F$. In fact,

$$
F \circ G(q)=F\left(\theta_{a-f(q)}(q), f(q)\right)=\theta_{f(q)-a} \circ \theta_{a-f(q)}(q)=\theta_{0}(q)=q,
$$

and

$$
\begin{aligned}
G \circ F(p, t) & =G\left(\theta_{t-a}(p)\right)=\left(\theta_{a-f\left(\theta_{t-a}(p)\right)}\left(\theta_{t-a}(p)\right), f\left(\theta_{t-a}(p)\right)\right)=\left(\theta_{t-f\left(\theta_{t-a}(p)\right)}(p), f\left(\theta_{t-a}(p)\right)\right) \\
& =(p, t),
\end{aligned}
$$

since $f\left(\theta_{t-a}(p)\right)=t-a+f(p)=t$. Then $F$ is a diffeomorphism. Furthermore, the diagram is commutative because $f \circ F(p, t)=f\left(\theta_{t-a}(p)\right)=t=\pi_{2}(p, t)$.

Example 2.14. Let $M \subset \mathbb{R}^{3}$ be the cylinder of radius 1 and height 1 above the plane $z=0$, see Figure 11. Let $h: M \rightarrow \mathbb{R}$ be the height function, then $\partial(M)=h^{-1}(0) \sqcup h^{-1}(1)$. Note that the function $h$ has no critical point and $M=h^{-1}([0,1])$. Then by previous theorem $M$ is diffeomorphic to $h^{-1}(0) \times[0,1]$. Since $h^{-1}(0)=S^{1}$, we have that $M$ is diffeomorphic to $S^{1} \times[0,1]$.

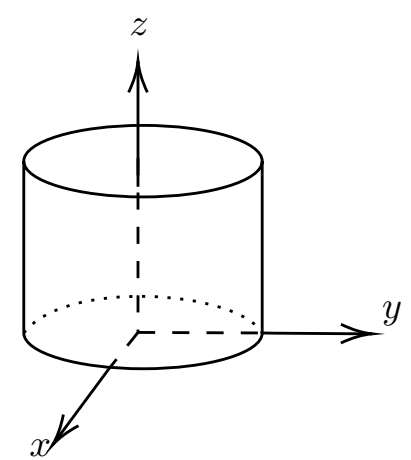

Figure 11 - Cylinder

Example 2.15. The above example can be generalized as follows. Let $M$ be a compact smooth manifold with boundary $\partial M=A \sqcup B$. Suppose that there exists a smooth function $h: M \rightarrow[a, b]$ such that $f^{-1}(a)=A$ and $f^{-1}(b)=B$ and there is no critical point in $f^{-1}([a, b])$. Then $M$ is diffeomorphic to $A \times[a, b]$.

Corollary 2.16 (Reeb's Theorem). Let $M$ be a compact $m$-manifold without boundary and let $f: M \rightarrow \mathbb{R}$ be a Morse function. Suppose that $f$ has only two critical points. Then $M$ is homeomorphic to $S^{m}$.

Proof. We will to construct a homeomorphism $G: M \longrightarrow S^{m}$. Let $p_{+}, p_{-} \in M$ be the two critical points of $f$. Since $M$ is compact, $f$ attains a maximum and a minimum. Suppose that $f$ attains a 
maximum at $p_{+}$and attains a minimum at $p_{-}$. Then by Lemma 2.2 and Example 2.5 we have that there exist chats $\left(U_{1}^{\prime}, \varphi_{1}\right)$ and $\left(V_{1}^{\prime}, \phi_{1}\right)$ around the points $p_{+}$and $p_{-}$respectively such that

$$
\begin{array}{lr}
f \circ \varphi_{1}^{-1}\left(x_{1}, \ldots, x_{m}\right)=f\left(p_{+}\right)-x_{1}^{2}-\cdots-x_{m}^{2}, & \text { for } x \in \varphi_{1}\left(U_{1}^{\prime}\right), \\
f \circ \phi_{1}^{-1}\left(x_{1}, \ldots, x_{m}\right)=f\left(p_{-}\right)+x_{1}^{2}+\cdots+x_{m}^{2}, & \text { for } x \in \phi_{1}\left(V_{1}^{\prime}\right) .
\end{array}
$$

Let $\varepsilon>0$ small enough such that $U_{1}:=f^{-1}\left(\left[f\left(p_{+}\right)-\varepsilon, f\left(p_{+}\right)\right]\right) \subset U_{1}^{\prime}$ and $V_{1}:=f^{-1}\left(\left[f\left(p_{-}\right), f\left(p_{-}\right)+\right.\right.$ $\varepsilon]) \subset V_{1}^{\prime}$. Then we have,

$$
M=U_{1} \cup W_{1} \cup V_{1},
$$

where $W_{1}:=f^{-1}\left(\left[f\left(p_{-}\right)+\varepsilon, f\left(p_{+}\right)-\varepsilon\right]\right)$. Note that

$$
\begin{aligned}
\varphi_{1}\left(U_{1}\right) & =\varphi_{1}\left(f^{-1}\left(\left[f\left(p_{+}\right)-\varepsilon, f\left(p_{+}\right)\right]\right)\right)=\left(f \circ \varphi_{1}\right)^{-1}\left(\left[f\left(p_{+}\right)-\varepsilon, f\left(p_{+}\right)\right]\right) \\
& =\left\{\left(x_{1}, \ldots, x_{m}\right) \in \mathbb{R}^{m} \mid f\left(p_{+}\right)-\varepsilon \leq f\left(p_{+}\right)-x_{1}^{2}-\cdots-x_{m}^{2} \leq f\left(p_{+}\right)\right\}=D_{\varepsilon}^{m} .
\end{aligned}
$$

By same argument we have $\phi_{1}\left(V_{1}\right)=D_{\varepsilon}^{m}$.

Now, let $h: S^{m} \rightarrow \mathbb{R}$ be the height function. Then, by Example 1.21 we have that the north pole $q_{+}$and the south pole $q_{-}$of $S^{m}$ are the maximal and minimal points of $h$ respectively.

We have that for (a possible new) $\varepsilon>0$ small enough there are charts $\left(U_{2}, \varphi_{2}\right)$ and $\left(V_{2}, \phi_{2}\right)$ around the points $q_{+}$and $q_{-}$respectively, where $U_{2}=h^{-1}\left(\left[h\left(q_{+}\right)-\varepsilon, h\left(q_{+}\right)\right]\right)$and $V_{2}=h^{-1}\left(\left[h\left(q_{-}\right), h\left(q_{-}\right)+\right.\right.$ $\varepsilon]$ ), such that

$$
\begin{array}{lr}
h \circ \varphi_{2}^{-1}\left(x_{1}, \ldots, x_{m}\right)=h\left(q_{+}\right)-x_{1}^{2}-\cdots-x_{m}^{2}, \\
h \circ \phi_{2}^{-1}\left(x_{1}, \ldots, x_{m}\right)=h\left(q_{-}\right)+x_{1}^{2}+\cdots+x_{m}^{2},
\end{array} \quad \text { for } x \in \varphi_{2}\left(U_{2}\right),
$$

Then

$$
S^{m}=U_{2} \cup W_{2} \cup V_{2},
$$

where $W_{2}:=h^{-1}\left[h\left(q_{-}\right)+\varepsilon, h\left(q_{+}\right)-\varepsilon\right]$. By the same argument above we have $\varphi_{2}\left(U_{2}\right)=\phi_{2}\left(V_{2}\right)=$ $D_{\varepsilon}^{m}$.

Now, we will construct homeomorphisms $G_{U}: U_{1} \rightarrow U_{2}, G_{W}: W_{1} \rightarrow W_{2}$, and $G_{V}: V_{1} \rightarrow V_{2}$ as follows. See Figure 12 below.

Let us define $G_{U}(x)=\varphi_{2}^{-1} \circ \varphi_{1}(x)$. Clearly $G_{U}$ is a homeomorphism and $G_{U}\left(f^{-1}\left(f\left(p_{+}\right)-\varepsilon\right)\right)=$ $h^{-1}\left(h\left(q_{+}\right)-\varepsilon\right)$.

Since there is no critical values of $f$ between $f\left(q_{+}\right)$and $f\left(q_{-}\right)$, by Example 2.15 there is a diffeomorphism

$$
\Psi_{1}: W_{1} \rightarrow f^{-1}\left(f\left(p_{+}\right)-\varepsilon\right) \times[0,1] .
$$

Similarly we have that there is a diffeomorphism

$$
\Psi_{2}: W_{2} \rightarrow h^{-1}\left(h\left(q_{+}\right)-\varepsilon\right) \times[0,1]
$$




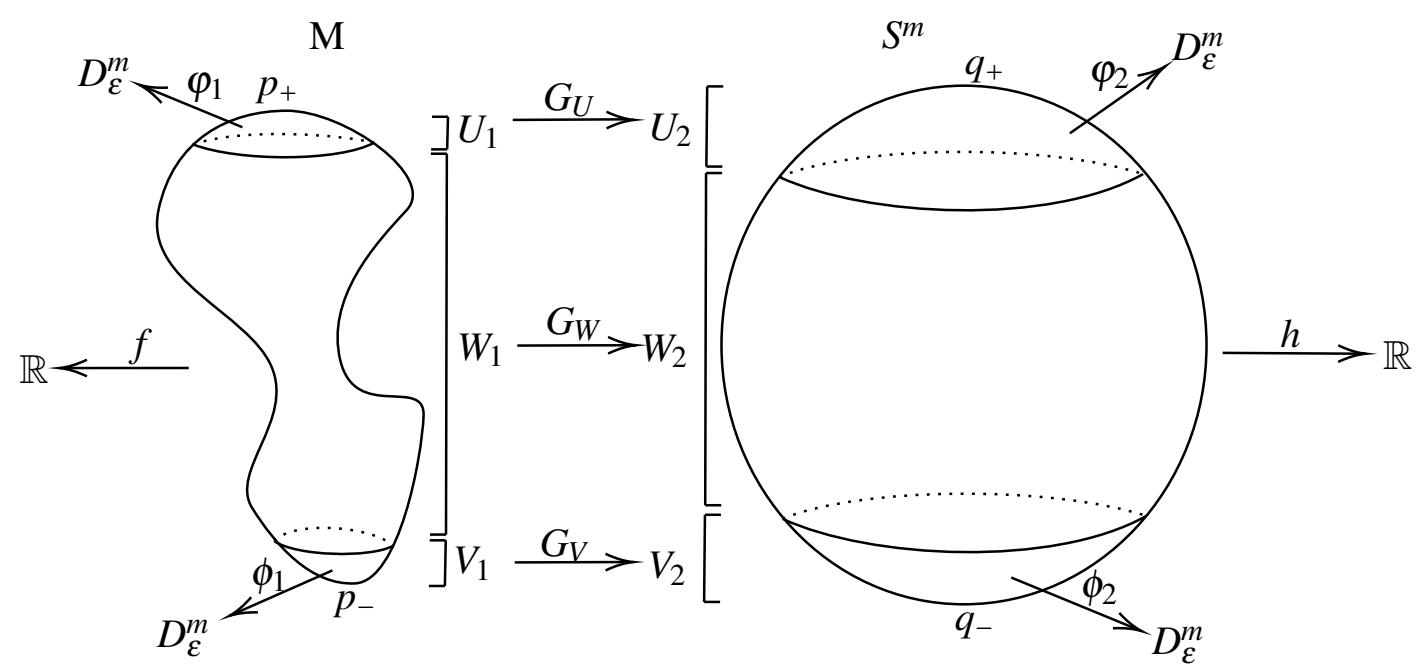

Figure 12 - Reeb's Theorem

Consider the following homeomorphism

$$
\begin{aligned}
G_{W}^{\prime}: f^{-1}\left(f\left(p_{+}\right)-\varepsilon\right) \times[0,1] & \longrightarrow h^{-1}\left(h\left(q_{+}\right)-\varepsilon\right) \times[0,1] \\
(x, t) & \longmapsto\left(G_{U}(x), t\right) .
\end{aligned}
$$

With this let us define $G_{W}=\Psi_{2}^{-1} \circ G_{W}^{\prime} \circ \Psi_{1}$. Note that $\phi_{1}\left(\partial V_{1}\right)=\partial D^{m}$. Since for $x \in D^{M}$ we have that $\varepsilon \frac{x}{\|x\|} \in \partial D^{m}$, the following function is well defined, see Figure 13 below.

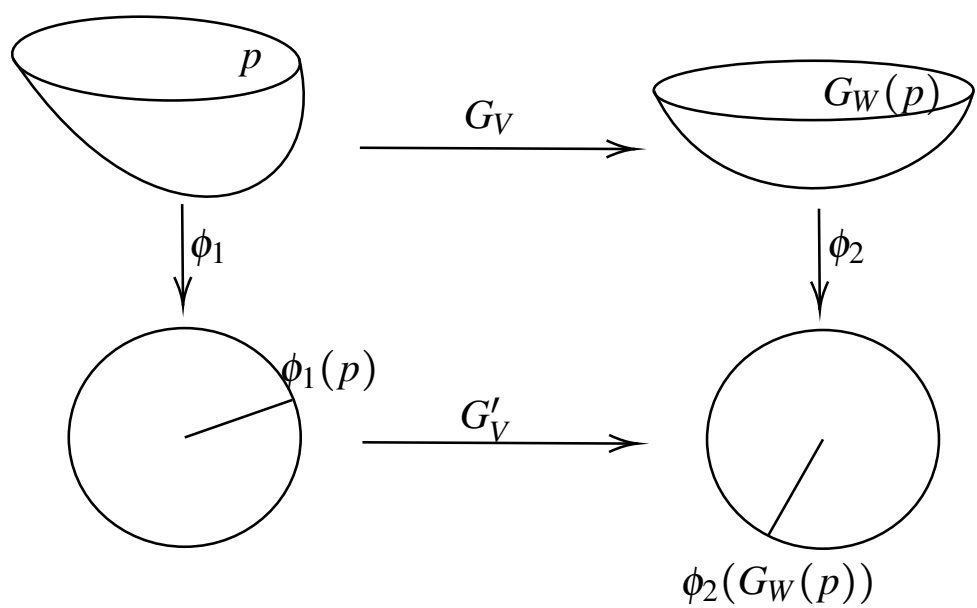

Figure $13-G_{V}$

$$
G_{V}^{\prime}: D^{m} \rightarrow D^{m}, x \mapsto\left\{\begin{array}{cc}
0 & \text { if } x=0, \\
\frac{\|x\|}{\varepsilon} \phi_{2} \circ G_{W} \circ \phi_{1}^{-1}\left(\varepsilon \frac{x}{\|x\|}\right) & \text { if } \quad x \neq 0 .
\end{array}\right.
$$

Let us define $G_{V}=\phi_{2} \circ G_{V}^{\prime} \circ \phi_{1}^{-1}$ which is a homeomorphism. Since the functions $G_{U}, G_{W}$ and $G_{V}$ are equal in the common domain the function $G: M \longrightarrow S^{m}$ defined by

$$
G(x)=\left\{\begin{array}{lll}
G_{U}(x) & \text { if } & x \in U_{1} \\
G_{W}(x) & \text { if } & x \in W_{1} \\
G_{V}(x) & \text { if } & x \in V_{1}
\end{array}\right.
$$


is also a homeomorphism, this finish the proof.

Remark 2.17. The Reeb's theorem holds even if the two critical points are degenerates, i.e., if $M$ is a closed manifold and $f: M \rightarrow \mathbb{R}$ a smooth function with only two critical points then $M$ is homeomorphic to $S^{n}$. Following (MILNOR, 1964, Theorem 1', Chapter 6) the idea to show that is as follows: let $p$ and $q$ be the critical point of $f$ and $U \subset M$ be an open set diffeomorphic to $\mathbb{R}^{n}$ such that $p \in U$ but $q \notin U$, given a compact set $K \subset M-\{q\}$ using the flow $\theta$ of the gradient vector field of $f$ we can find $t \in \mathbb{R}$ such that $K \subset \theta_{t}(U)$. This allows us to apply the Brown-Stalling Lemma (MILNOR, 1964, Chapter 6) which state that in a (paracompact) manifold $N$ such that every compact set is contained in an open set homeomophic to $\mathbb{R}^{n}$ is itself homoeomorphic to $\mathbb{R}^{n}$, hence $M-\{q\}$ is diffeomorphic to $\mathbb{R}^{n}$. Therefore we may use the stereographic projection and conclude that $M$ is homeomorphic to $S^{n}$.

\subsection{Morse functions and homotopy type}

In Theorem 2.13 it was shown that $M^{a}$ and $M^{b}$ are diffeomorphics if there is not critical values between $a$ and $b$. In particular, they have the same homotopy type. The following theorem describes the change of the homotopy type between $M^{a}$ and $M^{b}$ when there is a critical value between $a$ and $b$.

Theorem 2.18. Let $f: M \rightarrow \mathbb{R}$ be a smooth function on a manifold $M$ with a non-degenerate critical point $p$ of index $\lambda$ and $c=f(p)$. Suppose that there is a $\delta>0$ such that $f^{-1}([c-\delta, c+\delta])$ is compact and does not contains critical points other than $p$. Then there is a $0<\varepsilon<\delta$ such that $M^{c+\varepsilon}$ has the same homotopy type of $M^{c-\varepsilon}$ with $\lambda$-cell attached.

Proof. By Morse lemma there is a chart $\left(U, \varphi=\left(u_{1}, \ldots, u_{m}\right)\right)$ such that $u_{i}(p)=0$ and

$$
f\left(u_{1}, \ldots, u_{m}\right)=c-u_{1}^{2}-\cdots-u_{\lambda}^{2}+u_{\lambda+1}^{2}+\cdots+u_{m}^{2}
$$

Let $\varepsilon>0$ such that $f^{-1}([c-\varepsilon, c+\varepsilon])$ is compact and does not contains critical points other than $p$.

Define on $U$ the following two functions $\zeta=u_{1}^{2}+\cdots+u_{\lambda}^{2}$ and $\eta=u_{\lambda+1}^{2}+\cdots+u_{m}^{2}$, then $f=c-\zeta+\eta$ on $U$. Consider the $\lambda$-cell $e^{\lambda}=\{q \in U \mid \zeta(q) \leq \varepsilon$ and $\eta(q)=0\}$ then the boundary $\partial e^{\lambda}=\{q \epsilon$ $U \mid \zeta(q)=\varepsilon$ and $\eta(q)=0\}$ is equal to $e^{\lambda} \cap M^{c-\varepsilon}$. Hence, the $\lambda$-cell $e^{\lambda}$ is attached to $M^{c-\varepsilon}$ as required. It remains to show that $M^{c-\varepsilon} \cup e^{\lambda}$ is a deformation retract of $M^{c+\varepsilon}$.

We will construct a smooth function $F: M \rightarrow \mathbb{R}$ as follows. Let $\mu: \mathbb{R} \rightarrow \mathbb{R}$ be a smooth function satisfying the conditions

$$
\begin{array}{cl}
\mu(0)>\varepsilon & \\
\mu(r)=0 \quad \text { if } r \geq 2 \varepsilon \\
-1<\mu^{\prime}(r) \leq 0 & \text { for all } r \in \mathbb{R} .
\end{array}
$$




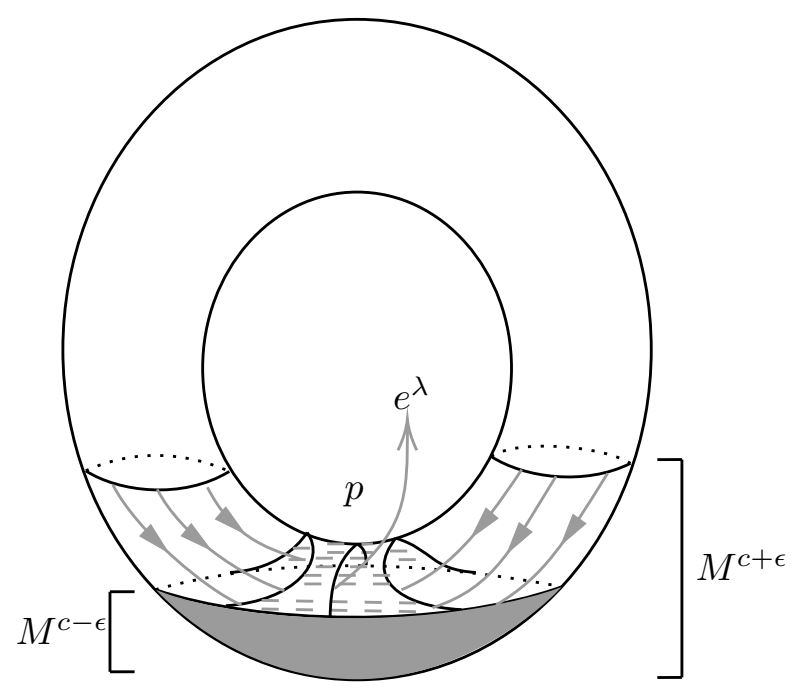

Figure 14

We can choose $\varepsilon>0$ such that $D_{(2 \varepsilon)^{1 / 2}}^{m} \subset \varphi(U)$ and define

$$
F(q)=\left\{\begin{array}{cll}
f(q) & \text { if } & q \notin \varphi^{-1}\left(D_{(2 \varepsilon)^{1 / 2}}^{m}\right) \\
f(q)-\mu(\zeta(q)+2 \eta(q)) & \text { if } & q \in U .
\end{array}\right.
$$

It is well defined because $\mu(\zeta(q)+2 \eta(q))=0$ on $\left(\varphi^{-1}\left(D_{(2 \varepsilon)^{1 / 2}}^{m}\right)\right)^{c} \cap U$. Now, we will show the following facts.

1. $F^{-1}((-\infty, c+\varepsilon])=M^{c+\varepsilon}$,

2. $\sum(f)=\sum(F)$ (i.e., $\{$ critical points of $f\}=\{$ critical points of $F\}$ ),

3. $F^{-1}((-\infty, c-\varepsilon])$ is a deformation retract of $M^{c+\varepsilon}$ (see Figure 17 and Figure 15),

4. $M^{c-\varepsilon} \cup e^{\lambda}$ is a deformation retract of $F^{-1}((-\infty, c-\varepsilon])$, (see Figure 16).

Proof of 1 . It is clear that $M^{c+\varepsilon} \subset F^{-1}((-\infty, c+\varepsilon])$ because $F \leq f$. Let $q \in F^{-1}((-\infty, c+\varepsilon])$. If $\zeta(q)+2 \eta(q) \geq 2 \varepsilon$ then $f(q)=F(q)$, if $\zeta(q)+2 \eta(q) \leq 2 \varepsilon$ then

$$
F(q) \leq f(q)=c-\zeta(q)+\eta(q) \leq c+\frac{1}{2} \zeta(q)+\eta(q) \leq c+\varepsilon
$$

hence, $q \in M^{c+\varepsilon}$.

Proof of 2. Since $F=f$ outside of $U$, it is only necessary to compute the critical points of 


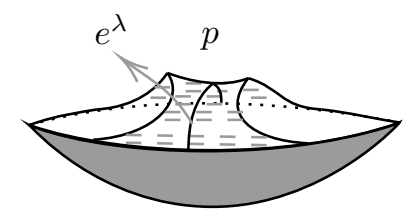

Figure $15-F^{-1}(-\infty, c-\varepsilon]$

$F$ on $U$. We have that,

$$
\begin{aligned}
d_{q} F & =\left(\frac{\partial F}{\partial u_{i}}(q)\right)_{i=1, \ldots, m} \\
& =\left(\frac{\partial \zeta}{\partial u_{i}}(q)+\frac{\partial \eta}{\partial u_{i}}(q)-\mu^{\prime}(\zeta(q)+2 \eta(q))\left(\frac{\partial \zeta}{\partial x_{i}}(q)+2 \frac{\partial \eta}{\partial x_{i}}(q)\right)\right) \\
& =\left(\left(-1-\mu^{\prime}(\zeta(q)+2 \eta(q))\right) \frac{\partial \zeta}{\partial u_{i}}(q)+\left(1-2 \mu^{\prime}(\zeta(q)+2 \eta(q))\right) \frac{\partial \eta}{\partial x_{i}}(q)\right) \\
& =\frac{\partial F}{\partial \zeta}(q) d_{q} \zeta+\frac{\partial F}{\partial \eta}(q) d_{q} \eta .
\end{aligned}
$$

Observe that

$$
\begin{aligned}
& \frac{\partial F}{\partial \zeta}=-1-\mu^{\prime}(\zeta+2 \eta) \leq-1 \\
& \frac{\partial F}{\partial \eta}=1-2 \mu^{\prime}(\zeta+2 \eta) \geq 1
\end{aligned}
$$

Hence, $d_{q} F$ is zero only when $d_{q} \zeta$ and $d_{q} \eta$ are both zero and that only happens at $p$. Therefore $F$ only has a critical point at $p$.

Proof of 3. By item 1. together with the inequality $F \leq f$ we have that $F^{-1}([c-\varepsilon, c+\varepsilon]) \subset$ $f^{-1}([c-\varepsilon, c+\varepsilon])$ and since $f^{-1}([c-\varepsilon, c+\varepsilon])$ is compact, $F^{-1}([c-\varepsilon, c+\varepsilon])$ is also compact. Since $F(p)=c-\mu(0) \leq c-\varepsilon$, we have that $p \notin F^{-1}([c-\varepsilon, c+\varepsilon])$. Since $p$ is the unique critical point of $F$ on $U, F^{-1}([c-\varepsilon, c+\varepsilon])$ does not has critical points of $F$. Therefore, by Theorem 2.13 $F^{-1}((-\infty, c-\varepsilon])$ is a deformation retract of $M^{c+\varepsilon}=F^{-1}((\infty, c+\varepsilon])$. See Figure 17 and Figure 15 .

Proof of 4. In order to emphasize the function $f$ denote $M_{f}^{a}=f^{-1}((-\infty, a])$. Define $H=\overline{M_{F}^{c-\varepsilon} \backslash M_{f}^{c-\varepsilon}}$.

Claim: $e^{\lambda} \subset H$.

Indeed, remind that $q \in H$ if and only if $F(q) \leq c-\varepsilon \leq f(q)$. Hence, if $q \in e^{\lambda}$ then $\zeta(q) \leq \varepsilon$ and $\eta(q)=0$. We know that $\frac{\partial F}{\partial \zeta}<0$ and that $0=\zeta(p) \leq \zeta(q)$, so

$$
F(q) \leq F(p)=c-\mu(0)<c-\varepsilon
$$

and $q \in M_{F}^{c-\varepsilon}$. On the other hand,

$$
f(q)=c-\zeta(q) \geq c-\varepsilon,
$$


therefore $q \in \overline{\left(M_{f}^{c-\varepsilon}\right)^{C}}$ and $q \in H$, this prove the claim.

Since $F^{-1}((\infty, c-\varepsilon])=M_{F}^{c-\varepsilon}=M_{f}^{c-\varepsilon} \cup H$, it is enough to prove that $M_{f}^{c-\varepsilon} \cup e^{\lambda}$ is a deformation retract of $M_{f}^{c-\varepsilon} \cup H$.

Now, we define the retraction $r: I \times\left(M_{f}^{c-\varepsilon} \cup H\right) \rightarrow M_{f}^{c-\varepsilon} \cup H$ as follows: if $q \notin U$ define $r(t, q)=q$, if $q \in U$ define

$$
r\left(t, q=\left(u_{1}, \ldots, u_{n}\right)\right)=\left\{\begin{array}{cll}
\left(u_{1}, \ldots, u_{\lambda}, t u_{\lambda+1} \ldots, t u_{n}\right) & \text { if } \zeta(q) \leq \varepsilon \\
\left(u_{1}, \ldots, u_{\lambda}, s_{t} u_{\lambda+1} \ldots, s_{t} u_{n}\right) & \text { if } \varepsilon \leq \zeta(q) \leq \eta(q)+\varepsilon \\
q & \text { if } \eta(q)+\varepsilon \leq \zeta(q)
\end{array}\right.
$$

where

$$
s_{t}=t+(1-t)\left(\frac{\zeta(q)-\varepsilon}{\eta(q)}\right)
$$

It only remains to verify that $r$ is well defined and that it is indeed a deformation retract.

On $U \cap M_{F}^{c-\varepsilon}$ we have:

- If $\zeta(q) \leq \varepsilon$ then $q \in H$ and $r_{t}(q)=r(t, q) \in H$. Indeed,

$$
f(q)=c-\zeta(q)+\eta(q) \geq c-\varepsilon+\eta(q) \geq c-\varepsilon,
$$

then $q \in H$. Note that $\frac{\partial F}{\partial \eta}>0, \eta\left(r_{t}(q)\right) \leq \eta(q)$ and $\zeta\left(r_{t}(q)\right)=\zeta(q)$ for all $t \in I$, then we have that $F\left(r_{t}(q)\right) \leq F(q) \leq c-\varepsilon$. Therefore $r_{t}(q) \in M_{F}^{c-\varepsilon}$ and $r_{t}(q) \in H$.

- If $\varepsilon \leq \zeta(q) \leq \eta(q)+\varepsilon$ (i.e., $c-\varepsilon \leq f(q)$ and $\zeta(q) \geq \varepsilon$ ), we also have that $q \in H$ because $f(q)=c-\zeta(q)+\eta(q) \geq c-\varepsilon$ and $\zeta(q) \leq s_{t}(q) \eta(q)+\varepsilon, r_{t}(q) \in H$.

If $\zeta(q)=\varepsilon$ then $s_{t}(q)=t$. So it coincides with the previous case. If $\eta(q)=0$ define $r_{t}(q)=\left(u_{1}, \ldots, u_{\lambda}, 0, \ldots, 0\right)$ that is because

$$
\lim _{\eta(q) \rightarrow 0} \frac{\zeta(q)-\varepsilon}{\eta(q)} u_{i}=0, \quad i=\lambda+1, \ldots, n
$$

indeed, $0 \leq \frac{\zeta(q)-\varepsilon}{\eta(q)} u_{i} \leq u_{i}$ and $\lim _{\eta(q) \rightarrow 0} u_{i}=0$.

- when $\eta(q)+\varepsilon \leq \zeta(q)$ clearly $r(t, q)$ is well defined. If $\eta(q)+\varepsilon=\zeta(q)$ then $r_{t}(q)$ coincides with the previous case.

Therefore, the retraction $r_{t}$ is well defined and $r_{0}\left(M_{f}^{c-\varepsilon} \cup H\right)=M_{f}^{c-\varepsilon} \cup e^{\lambda}$.

Corollary 2.19. Under the hypothesis of the previous theorem we also have that the set $M_{f}^{c}$ is a deformation retract of $M^{c+\varepsilon}$.

Proof. Just take $\varepsilon=0$ in the definition of the retraction $r$ in previous proof. Then $H=\overline{M_{F}^{c} \backslash M_{f}^{c}}$, and 


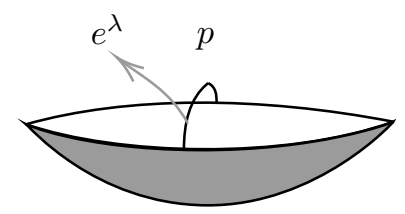

Figure $16-M^{c-\varepsilon} \cup e^{\lambda}$

$$
r\left(t, q=\left(u_{1}, \ldots, u_{n}\right)\right)=\left\{\begin{array}{cl}
\left(u_{1}, \ldots, u_{\lambda}, s_{t} u_{\lambda+1} \ldots, s_{t} u_{n}\right) & \text { if } q \in H, \\
q & \text { if } q \in M_{f}^{c}
\end{array}\right.
$$

where

$$
s_{t}=\frac{\zeta(q)}{\eta(q)}
$$

One of the hypothesis of the previous theorem is that $p$ is the unique critical point on $f^{-1}(c)$. Since the previous proof is on a small neighborhood of $p$, a similar argument proves the following corollary.

Corollary 2.20. If the level set $f^{-1}(c)$ has $k$ critical points $p_{1}, \ldots, p_{k}$, with indices $\lambda_{1}, \ldots, \lambda_{k}$ respectively, then $M^{c+\varepsilon}$ has the same homotopy type of $M^{c-\varepsilon}$ with a $\lambda_{i}$-cell attached for each $i=1 \ldots, k$.

It is possible adapt the techniques of previous theorem to prove that every manifold has the homotopy type of a CW-complex.

Theorem 2.21 (Homotopy type through Morse function). Let $f: M \rightarrow \mathbb{R}$ be a Morse function such that the critical points of $f$ are contained in $M^{t_{0}}$ for some $t_{0} \in \mathbb{R}$. Suppose that $M^{t}$ is compact for all $t \in \mathbb{R}$, then $M$ has the same homotopy type of a CW-complex $X$ with one cell of dimension $\lambda$ for each critical point of index $\lambda$.

Example 2.22. In Example 2.9 we show that the function

$$
\begin{aligned}
f: \mathbb{C} P^{n} & \longrightarrow \mathbb{R} \\
{\left[z_{0}, \ldots, z_{n}\right] } & \longmapsto \sum_{k=1}^{n} k\left|z_{k}\right|^{2}
\end{aligned}
$$

is a Morse function and we prove that $f$ has $n+1$ critical points $\left\{p_{0}, \ldots, p_{n}\right\}$ with indices $\lambda_{p_{j}}=2 j$ for $j=0, \ldots, n$.. Hence, by Theorem $2.21 \mathbb{C} P^{n}$ has the same homotopy type of a $\mathrm{CW}$-complex $X$ with one $2 j$-cell attached for each critical point $p_{j}$ with $j=0, \ldots, n$. By Theorem 1.38 we have that the homology groups of $\mathbb{C} P^{n}$ are isomorphic to the homology groups of the following chain 
complex

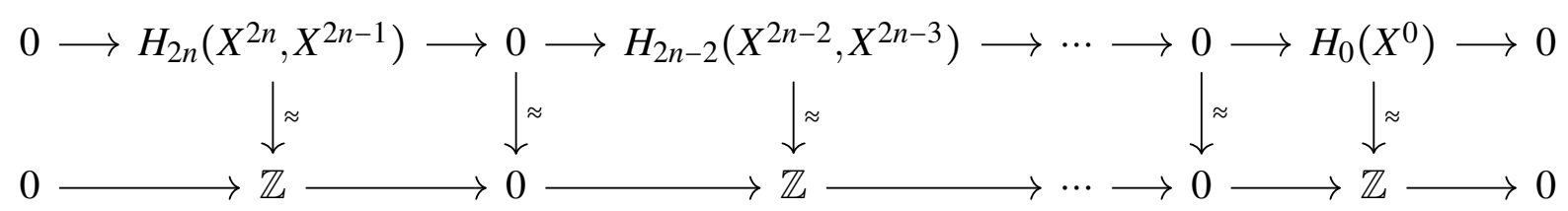

Therefore,

$$
H_{k}\left(\mathbb{C} P^{n}\right)=\left\{\begin{array}{lll}
\mathbb{Z} & \text { if } k \leq 2 n \text { and } k \text { even } \\
0 & \text { if } \text { otherwise }
\end{array}\right.
$$

\subsection{Morse and Poincaré Polynomials}

Classically is known that for $k$ a vector space, $X$ a topological space with $\operatorname{dim}_{k} H_{n}(X ; k)<$ $\infty$, the Poincaré series of $X$ is defined by $P_{X}(t)=\sum_{n=0}^{\infty} \operatorname{dim} H_{n}(X ; k) t^{n}$ and hence when $X$ is a finite $\mathrm{CW}$-complex the series becomes a polynomial. In general, for a sequence of $k$-vector spaces $V=\left(V_{0}, V_{1}, V_{2}, \ldots, V_{n}, \ldots\right)$ the formal Hilbert series is defined by $H_{t}(V)=\sum_{n=0}^{\infty} \operatorname{dim}_{k}\left(V_{n}\right) t^{n}$. In particular, considering $N$ is a $R$-module where $(R, \mathfrak{m})$ is a Noetherian local ring and the sequence of $\left(\frac{R}{\mathfrak{m}}\right)$-vector spaces $V=\left(\frac{\mathfrak{m}^{n} N}{\mathfrak{m}^{n+1} N}: n=0,1, \ldots\right)$, then a famous Hilbert's result state that there exists a polynomial $Q(t)$ with integer coefficients such that $H_{t}(V)=\frac{Q(t)}{(1-t)^{d}}$, where $d$ is the Krull dimension of $N$. For more details see (ANICK, 1988).

These definitions shows that the Poincaré series is a particular case of the Hilbert series. This may explain the fact that this type of series is usually called a Hilbert-Poincare series.

In this section we will present the Morse Inequalities, the Morse and Poincare polynomials and their relations. For that we remind Theorem 2.21 which state that a compact manifold $M$ has the same homotopy type of a CW-complex $X$ and by Lemma 1.37 we also have that $\operatorname{rank}\left(H_{k}\left(X^{k}, X^{k-1}\right)\right)=v_{k}$, where $v_{k}$ is the number of critical points of $f$ of index $k$. Hence, by Theorem 1.38 we have that $\operatorname{rank} H_{k}\left(X^{k}, X^{k-1}\right) \geq b_{k}$ where $b_{k}$ is the $k^{\text {th }}$ betti number, i.e., $b_{k}=\operatorname{rank}\left(H_{k}(M)\right)$. So, we obtain the following inequalities called weak Morse inequalities

$$
\begin{gathered}
v_{k} \geq b_{k}, \\
\sum_{k=0}^{m} v_{k} \geq \sum_{k=0}^{m} b_{k} .
\end{gathered}
$$

Theorem 2.23 (Morse inequalities). Let $M$ be a manifold $m$-dimensional and $f: M \rightarrow \mathbb{R}$ be a Morse function, then

1.

$$
\sum_{k=0}^{n}(-1)^{k+n} v_{k} \geq \sum_{k+0}^{n}(-1)^{k+n} b_{k}
$$

for every $n=0, \ldots, m$. 
2.

$$
\sum_{k=0}^{m}(-1)^{k} v_{k}=\sum_{k=0}^{m}(-1)^{k} b_{k}=: \chi(M)
$$

where $v_{k}$ is the number of critical points of $f$ of index $k$.

For the proof of Theorem 2.23 we will need the following theorem from homological algebra.

Theorem 2.24. Let $\left(C_{*}, \partial_{*}\right)$ be a finitely generated chain complex, assume that $C_{k}=0$ for $k>m$. Let $c_{k}=\operatorname{rank}\left(C_{k}\right)$ and $b_{k}=\operatorname{rank}\left(H_{k}\left(C_{*}\right)\right)$ for all $k=0, \ldots, m$. Then

$$
\sum_{k=0}^{m}(-1)^{k} c_{k}=\sum_{k=0}^{m}(-1)^{k} b_{k}
$$

Proof. From the following two exact sequences

$$
\begin{gathered}
0 \rightarrow \operatorname{ker} \partial_{k} \rightarrow C_{k} \rightarrow \operatorname{Im} \partial_{k} \rightarrow 0, \\
0 \rightarrow \operatorname{Im} \partial_{k+1} \rightarrow \operatorname{ker} \partial_{k} \rightarrow H_{k} \rightarrow 0,
\end{gathered}
$$

we have that $c_{k}=\operatorname{rankker} \partial_{k}+\operatorname{rank} \operatorname{Im} \partial_{k}$ and $b_{k}=\operatorname{rankker} \partial_{k}-\operatorname{rank} \operatorname{Im} \partial_{k+1}$ for all $k=0, \ldots, m$. Then

$$
\sum_{k=0}^{m}(-1)^{k}\left(c_{k}-\operatorname{rank} \operatorname{Im} \partial_{k}\right)=\sum_{k=0}^{m}(-1)^{k}\left(b_{k}+\operatorname{rank} \operatorname{Im} \partial_{k+1}\right)
$$

Therefore,

$$
\begin{gathered}
\sum_{k=0}^{m}(-1)^{k} c_{k}=\sum_{k=0}^{m}(-1)^{k} b_{k}+\sum_{k=0}^{m}(-1)^{k+1}\left(\operatorname{rank} \operatorname{Im} \partial_{k}+\operatorname{rank} \operatorname{Im} \partial_{k+1}\right), \\
\sum_{k=0}^{m}(-1)^{k} c_{k}=\sum_{k=0}^{m}(-1)^{k} b_{k}-\operatorname{rank} \operatorname{Im} \partial_{0}+(-1)^{m+1} \operatorname{rank} \operatorname{Im} \partial_{m+1} .
\end{gathered}
$$

Since $\operatorname{Im} \partial_{0}=\operatorname{Im} \partial_{m+1}=0$, we have that

$$
\sum_{k=0}^{m}(-1)^{k} c_{k}=\sum_{k=0}^{m}(-1)^{k} b_{k} .
$$

Now we are able to proof the Morse inequalities

Proof of the Theorem 2.23. We have that $H_{k}\left(X^{k}, X^{k-1}\right)=0$ for $k>m$. Then the chain complex $\left(H_{k}\left(X^{n}, X^{k-1}\right), \bar{\partial}_{n}\right)$ satisfies the previous lemma. Since $v_{k}=\operatorname{rank} H_{k}\left(X^{n}, X^{n-1}\right)$,

$$
\sum_{k=0}^{m}(-1)^{k} v_{k}=\sum_{k=0}^{m}(-1)^{k} b_{k}(R)=\chi(M)
$$


that shows the part (2).

For part (1), consider the chain complex $\left(H_{k}^{n}\left(X^{k}, X^{k-1}\right), \bar{\partial}_{k}\right)$, where

$$
H_{k}^{n}\left(X^{k}, X^{k-1}\right)=\left\{\begin{array}{cl}
H_{k}\left(X^{k}, X^{k-1}\right) & \text { if } k \leq n \\
0 & \text { if } k>n
\end{array}\right.
$$

Then, by previous theorem,

$$
\sum_{k=0}^{n}(-1)^{k} v_{k}=\sum_{k=0}^{n-1}(-1)^{k} b_{k}(R)+(-1)^{n} \operatorname{rank}\left(\frac{\operatorname{ker} \bar{\partial}_{n}}{\operatorname{Im} \bar{\partial}_{n+1}}\right) .
$$

Since $\operatorname{Im} \partial_{n+1}=0$ and $\operatorname{rankker} \bar{\partial}_{n} \geq b_{n}(R)$, we have that

$$
(-1)^{n} \sum_{k=0}^{n}(-1)^{k} v_{k} \geq(-1)^{n} \sum_{k=0}^{n}(-1)^{k} b_{k}(R)
$$

As an application of Morse inequalities we can prove the following classical result of algebraic topology.

Corollary 2.25. Let $M$ be a compact closed manifold of odd dimension, then $\chi(M)=0$, i.e., the Euler characteristic is zero.

Proof. Let $f: M \rightarrow \mathbb{R}$ be a Morse function. Denote by $v_{k}(f)$ and $v_{k}(-f)$ the number of critical Morse points of index $k$ of $f$ and $-f$, respectively. If $m$ is the dimension of $M$ it is clear that $v_{k}(f)=v_{m-k}(-f)$, then

$$
\begin{aligned}
\chi(M) & =\sum_{k=0}^{m}(-1)^{k} v_{k}(f) \\
& =\sum_{k=0}^{m}(-1)^{k} v_{m-k}(-f) \\
& =(-1)^{m} \sum_{k=0}^{m}(-1)^{m-k} v_{m-k}(-f) \\
& =(-1)^{m} \sum_{i=0}^{m}(-1)^{i} v_{i}(-f) \\
& =(-1)^{m} \chi(M),
\end{aligned}
$$

since $m$ is odd, $\chi(M)=0$.

Now, we will present an equivalent perspective of the Morse inequalities through Poincare and Morse polynomials.

Definition 2.26. Let $M$ be a compact manifold $m$-dimensional, and $f: M \rightarrow \mathbb{R}$ be a Morse function. The Poincare polynomial of $M$ is defined to be

$$
P_{t}(M)=\sum_{k=0}^{m} b_{k} t^{k}
$$


and the Morse polynomial of $f$ is defined to be

$$
M_{t}(f)=\sum_{k=0}^{m} v_{k} t^{k}
$$

Theorem 2.27. Let $M$ be a compact manifold $m$-dimensional and $f: M \rightarrow \mathbb{R}$ be a Morse function. Then,

$$
M_{t}(f)=P_{t}(M)+(1+t) R(t)
$$

where $R(t)$ is a polynomial with non-negative integer coefficients.

Since we already proved Theorem 2.23 the following Proposition proves Theorem 2.27.

Proposition 2.28. Theorem 2.23 and Theorem 2.27 are equivalent.

Proof. Suppose that Theorem 2.23 holds, then by part (2),

$$
P_{-1}(M)=\sum_{k=0}^{m}(-1)^{k} b_{k}=\sum_{k=0}^{m} v_{k}=M_{-1}(f),
$$

thus $P_{-1}(M)-M_{-1}(f)=0$ therefore $(1+t)$ divides $P_{t}(M)-M_{t}(f)$. Then, there is a polynomial $R(t)=\sum_{n=0}^{m-1} r_{n} t^{n}$ such that $P_{t}(M)-M_{t}(f)=(1+t) R(t)$, clearly $r_{n} \in \mathbb{Z}$ because both $M_{t}(f)$ and $P_{t}(f)$ have integer coefficients. Now we will show that part (1) of Theorem 2.23 is equivalent to the fact that $r_{n} \geq 0$ for all $n=0, \ldots, m-1$. To see this just compare the coefficients. Indeed, since $M_{t}(f)=P_{t}(M)+(1+t) R(t)$,

$$
\begin{aligned}
v_{0} & =b_{0}+r_{0} \\
v_{1} & =b_{1}+r_{1}+r_{0} \\
& \vdots \\
v_{n} & =b_{n}+r_{n-1}+r_{n} .
\end{aligned}
$$

Thus,

$$
v_{n}-v_{n-1}+\cdots+(-1)^{n} v_{0}=b_{n}-b_{n-1}+\cdots+(-1)^{n} b_{0}+r_{n}
$$

Since

$$
\sum_{k=0}^{n}(-1)^{k+n} v_{k}-\sum_{k=0}^{n}(-1)^{k+n} b_{k} \geq 0
$$

we have that $r_{n} \geq 0$. Therefore Theorem $2.23 \Rightarrow$ Theorem 2.27.

Now suppose that Theorem 2.27 holds, i.e., assume that $M_{t}(f)=P_{t}(M)+(1+t) R(t)$ where $R(t)=\sum_{k=1}^{m-1} r_{k} t^{k}$ with $r_{k}$ non-negative integer for $k=0, \ldots, m-1$. Then as above we have

$$
v_{n}-v_{n-1}+\cdots+(-1)^{n} v_{0}=b_{n}-b_{n-1}+\cdots+(-1)^{n} b_{0}+r_{n},
$$

for all $n=0, \ldots, m-1$, since $r_{n} \geq 0$,

$$
\sum_{k=0}^{n}(-1)^{k+n} v_{k} \geq \sum_{k+0}^{n}(-1)^{k+n} b_{k}
$$

for every $n=0, \ldots, m$. This shows part (1). For part (2) it is just put $t=-1$. Therefore Theorem $2.27 \Rightarrow$ Theorem 2.23. 


\subsubsection{Perfect Morse functions and examples}

By definition a Morse function $f$ on a manifold $M$ is said a perfect Morse function if the Poincare and Morse polynomials are equal. In particular, the coefficients of those polynomials are equal $\left(v_{k}=b_{k}\right)$ and hence the weak Morse inequalities are equalities. The called Morse's Lacunary principle says that if the Morse polynomial has no consecutive powers of $t$, then the respective Morse function is perfect. For existence of perfect Morse functions the reader may see (ANDRICA; ALDEA, 2004). To finish this section we will give some example of perfect Morse functions and no perfect Morse function.

Example 2.29. In Example 2.8 we show that $f: T^{2} \rightarrow \mathbb{R},(x, y) \mapsto \cos (2 \pi x)+\cos (2 \pi y)$ is a Morse function which has one critical point of index 0 , two critical points of index 1 and one critical point of index 2. Moreover, in Example 1.22 we calculate the homology group of $T^{2}$. Hence, we have that

$$
\begin{gathered}
M_{t}(f)=t^{2}+2 t+1 \\
P_{t}(M)=t^{2}+2 t+1 .
\end{gathered}
$$

Therefore $M_{t}(f)=P_{t}(M)$.

Example 2.30. Consider the height function on the sphere $f: S^{2} \subset \mathbb{R}^{3} \rightarrow \mathbb{R},(x, y, z) \mapsto z$. In Example 1.21 we saw that the map $f$ has only two critical point: $p=(0,0,-1)$ a minimum with index 0 and $q=(0,0,1)$ a maximum with index 2. Moreover, in Example 1.39 the homology groups of $S^{2}$ was calculated and therefore

$$
M_{t}(f)=t^{2}+1=P_{t}\left(S^{2}\right)
$$

A similar argument can be used for the $n$-sphere $S^{n}$, to show that $M_{t}(f)=t^{n}+1=P_{t}\left(S^{n}\right)$, for all $n \geq 1$.

Example 2.31. Consider the function $f$ on $\mathbb{C} P^{n}$ defined in Example 2.9. We saw that $f$ has one critical point of index $2 k$ for $k=0, \ldots, n$. Moreover, in Example 2.22 was calculated the homology groups of $\mathbb{C} P^{n}$, then

$$
M_{t}(f)=t^{2 n}+t^{2 n-2}+\cdots+t^{2 n-2 i}+\cdots++t^{2}+1=P_{t}\left(\mathbb{C} P^{n}\right) .
$$

We will illustrate one example of non perfect Morse function.

Example 2.32. Consider $M=S^{2}$ the deformed sphere tangent to the plane $z=0$ and the height function $h: M \rightarrow \mathbb{R}$. Then $f$ has 4 critical points: the point $p$ of index 0 , the point $q$ of index 1 and the points $r, s$ of index 2. See Figure below. 


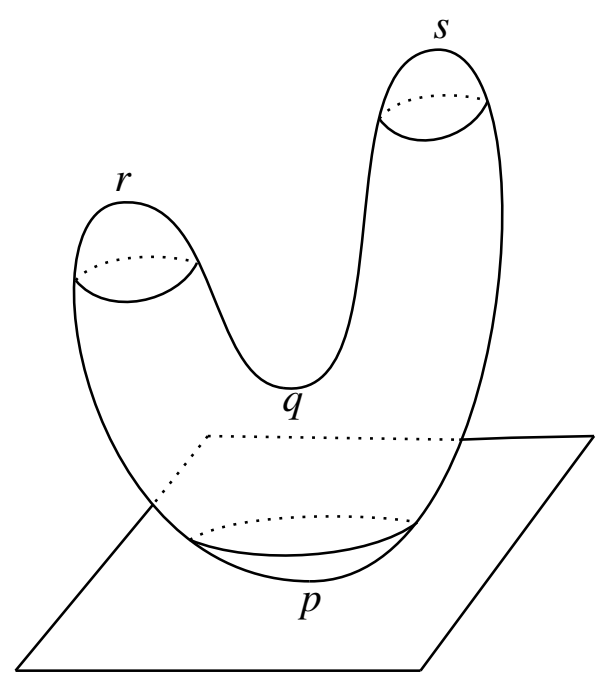

Figure 17

Hence, the Morse polynomial of $F$ is $M_{t}(h)=2 t^{2}+t+1$ and since $P_{t}\left(S^{2}\right)=t^{2}+1$ we conclude that $h$ is non perfect Morse function and $M_{t}(h)=P_{t}\left(S^{2}\right)+(1+t) t$. 

CHAPTER

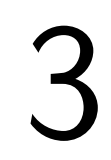

MORSE HOMOLOGY

Given $f: M \rightarrow \mathbb{R}$ a Morse function in Theorem 2.21 we saw that $M$ has the same homotopy type of a CW-complex $X$ with one cell of dimension $\lambda_{q}$ for each critical point $q$ of $f$ of Morse index $\lambda_{q}$. By Theorem 1.38 the homology of the chain complex (3.1) below is isomorphic to the singular homology of $M$.

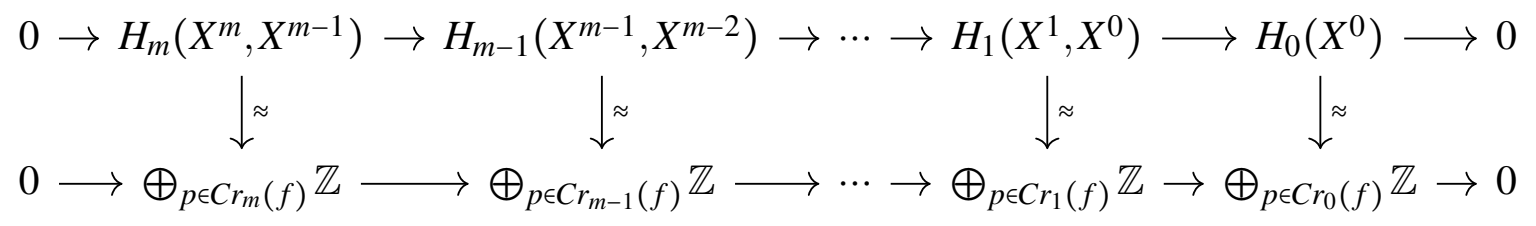

In order to know the homology groups of the manifold $M$, we need to study the attaching maps which give us the boundary operator of the chain complex (3.1). The first approach in order to give a formula for the boundary operator was made by Thom, Smale and Milnor. The Morse Homology Theorem gives a method to calculate the boundary operator of the chain complex (3.1).

The main goal of this chapter is to provide the necessary tools to introduce the MorseSmale-Witten chain complex and the Morse Homology Theorem. For this, we will study the Morse-Smale functions and show that for these types of functions have finitely many flow lines between two critical points of consecutive index of the negative gradient vector field.

Throughout this chapter we will work with a Riemannian compact smooth manifold that for the sake of simplicity we just denote by $M$.

The main reference for this chapter is (BANYAGA; HURTUBISE, 2013).

\subsection{Stable and Unstable Manifolds}

Given a Morse function $f$ on a compact manifold $M$ by Corollary 1.16 we have that the vector field $-\nabla f$ is complete. Throughout this section the map $\phi: \mathbb{R} \times M \rightarrow M$ denotes the flow 
generated of $-\nabla f$. We remind the notations for the flow lines,

$$
\phi^{p}: \mathbb{R} \rightarrow M, \phi^{p}(t)=\phi(t, p)
$$

and for the diffeomophisms of the flow,

$$
\phi_{t}: M \rightarrow M, \phi_{t}(p)=\phi(t, p)
$$

Proposition 3.1. Given $f: M \rightarrow \mathbb{R}$ a Morse function, then $f$ is nonincreasing along its gradient flow lines and strictly decreasing along the flow lines such that do not contain critical points, i.e., if $\phi^{p}$ is a flow line then $\phi^{p} \circ f$ is nondecreasing and strictly decreasing if $\operatorname{Im}\left(\phi^{p}\right)$ does not contain critical points.

Proof. Given a regular point $p \in M$ and $\phi^{p}: \mathbb{R} \rightarrow M$ its flow line, we have that

$$
\begin{aligned}
\frac{d}{d t} f\left(\phi^{p}(t)\right) & =d_{\phi^{p}(t)} f \circ \frac{d}{d t} \phi^{p}(t) \\
& =g\left(\nabla f\left(\phi^{p}(t)\right),-\nabla f\left(\phi^{p}(t)\right)\right) \\
& =-\mid\left(\left.\nabla f\left(\phi^{p}(t)\right)\right|_{g} ^{2} \leq 0\right.
\end{aligned}
$$

and $\nabla f$ is zero only at the critical points.

The following theorem shows that the flow lines of a Morse function always go from critical points to critical points.

Proposition 3.2. Let $f: M \rightarrow \mathbb{R}$ be a Morse function. Then, the limits

$$
\lim _{t \rightarrow \infty} \phi^{p}(t) \text { and } \lim _{t \rightarrow-\infty} \phi^{p}(t)
$$

converge to critical points of $f$ for all $p \in M$.

Proof. Since $M$ is compact and the image of $f \circ \phi^{p}$ is bounded,

$$
\lim _{t \rightarrow \pm \infty} \frac{d}{d t}\left(f \circ \phi^{p}\right)(t)=\lim _{t \rightarrow \pm \infty}-\left|\nabla\left(\phi^{p}(t)\right)\right|_{g}^{2}=0 .
$$

Since $M$ is compact and $f$ is a Morse function, $f$ has finite critical points. Define $U=\bigsqcup_{i \in J}=U_{i}$, where $J$ is in one-to-one correspondence with the critical points of $f$ and $U_{i}$ is an open ball for each critical point of $f$. Note that $M-U$ is compact then we can define $r=\min \left\{|\nabla f(x)|^{2} \mid x \epsilon\right.$ $M-U\}$. Since $M-U$ has no critical point, $r>0$. By the limit above, for $\varepsilon=r$ there is $t_{0} \in \mathbb{R}$ such that for all $t>t_{0}$ we have that $\left|\nabla f\left(\phi_{x}(t)\right)\right|^{2}<\varepsilon$. Therefore $\phi^{x}(t) \in U$ for all $t>0$. Since $\phi^{x}$ is continuous, $\phi^{x}(t) \in U_{i}$ for some $i \in J$. Note that the choice of $U_{i}$ was arbitrary. Then, if $q$ is the critical point on $U_{i}, \lim _{t \rightarrow \infty} \phi^{x}(t)=q$.

Definition 3.3. Let $p \in M$ be a non-degenerate critical point of $f$. 
1. The set

$$
W^{s}(p)=\left\{x \in M \mid \lim _{t \rightarrow \infty} \phi_{t}(x)=p\right\}
$$

is called the stable manifold of $p$.

2. The set

$$
W^{u}(p)=\left\{x \in M \mid \lim _{t \rightarrow-\infty} \phi_{t}(x)=p\right\}
$$

is called the unstable manifold of $p$.

Theorem 3.4 (Stable and Unstable Manifold Theorem). Let $f: M \rightarrow \mathbb{R}$ be a Morse function and $p$ be a critical point of $f$ of index $\lambda$. Then, the unstable and stable manifolds are smooth submanifolds diffeomorphic to a open $\lambda$-disk and $(n-\lambda)$-disk, respectively.

Moreover,

$$
T_{p} M=T_{p} W^{s}(p) \oplus T_{p} W^{u}(p),
$$

where the Hessian of $f$ at $p$ is negative definite on $T_{p} W^{u}(p)$ and positive definite on $T_{p} W^{s}(p)$.

Example 3.5. Consider the height function on the 2-sphere as in Example 1.21, see Figure 18. We have that the poles $p$ and $q$ are non-degenerates critical points.

$$
\begin{aligned}
W^{u}(q) & =S^{2}-\{p\} & W^{s}(q) & =\{q\} \\
W^{u}(p) & =\{p\} & W^{s}(p) & =S^{2}-\{q\}
\end{aligned}
$$

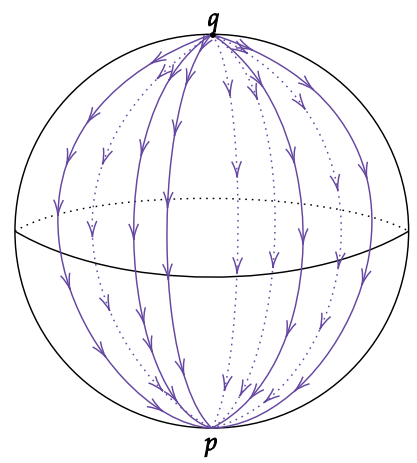

Figure $18-S^{2}$.

Example 3.6. Consider the function $f: T^{2} \rightarrow \mathbb{R}$ where $f(x, y)=\cos (2 \pi x)+\cos (2 \pi y)$ as in Example 2.8. We know that the points $p, q, r, s \in T^{2}$ are the critical points of $f$ of index $0,1,1$ and 2 respectively. The unstable manifold $W^{u}(r)$ is a circle through the points $r$ and $p$ minus the point $p$, the stable manifold $W^{s}(r)$ is a circle through the points $r$ and $s$ minus the point $s$. The unstable manifold $W^{u}(q)$ is a circle through the points $q$ and $p$ minus the point $p$, the stable manifold $W^{s}(q)$ is a circle through the points $q$ and $s$ minus the point $s$. The unstable manifold $W^{u}(s)=T^{2} \backslash W^{u}(r) \cup W^{u}(q) \cup W^{u}(p)$ and $W^{s}(s)=\{s\}$. 


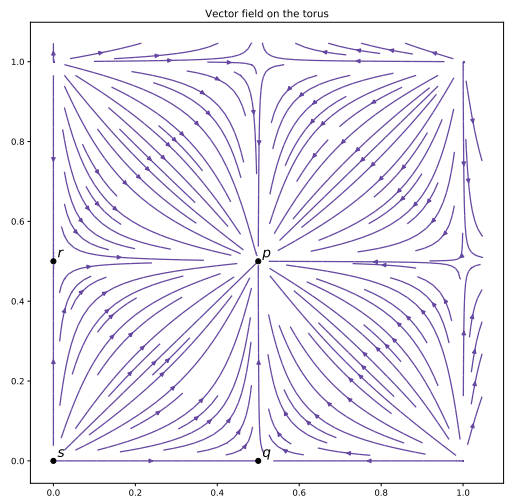

(a) $\mathbb{R}^{2} / \mathbb{Z}^{2}$.

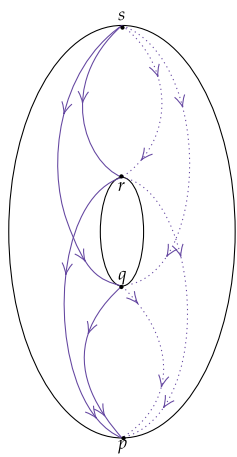

(b) $T^{2}$.

Figure 19 - Vector field on the Torus

Note that $\overline{W^{u}(s)}=W^{u}(s) \sqcup W^{u}(r) \sqcup W^{u}(q) \sqcup W^{u}(p)=T^{2}$, this can be generalized for Morse-Smale functions as we state in Proposition 3.17.

\subsubsection{Morse-Smale functions}

Definition 3.7. A Morse function $f: M \rightarrow \mathbb{R}$ satisfies the Morse-Smale transversality condition if for all critical points $p$ and $q$ of $f$ we have that $W^{u}(p)$ is transverse to $W^{s}(q)$. A smooth function $f$ is called a Morse-Smale function if it satisfies the Morse-Smale transversality condition. We denote the intersection $W^{u}(q) \cap W^{s}(p)$ by $W(q, p)$.

The Kupka-Smale Theorem states that the space of the Morse-Smale functions is a dense subspace of $C^{\infty}(M)$. See (BANYAGA; HURTUBISE, 2013) for a possible reference. Moreover, as a consequence of Morse-Smale transversality condition we have that $W(q, p)$ is a smooth manifold or empty and by Theorem 1.44 we can compute its dimension as follows.

Proposition 3.8. Let $f: M \rightarrow \mathbb{R}$ be a Morse-Smale function, $p$ and $q$ be a critical points of $f$ such that $W^{u}(q) \cap W^{s}(p) \neq \varnothing$. Then $W^{u}(q) \cap W^{s}(p)$ is an embedded submanifold of $M$ of dimension $\lambda_{q}-\lambda_{p}$, where $\lambda_{p}$ and $\lambda_{q}$ are the Morse index of $f$ at $p$ and $q$, respectively.

Proof. By Stable and Unstable Manifold Theorem we have that $W^{u}(q)$ and $W^{s}(p)$ are embedded submanifolds of $M$ of dimensions $\lambda_{q}$ and $m-\lambda_{p}$, respectively. Since the intersection is transversal and not empty, $W(q, p)$ is a embedded submanifold of $M$ and

$$
\begin{aligned}
\operatorname{dim}\left(W^{u}(q) \cap W^{s}(p)\right) & =\operatorname{dim} W^{u}(q)+\operatorname{dim} W^{s}(p)-m \\
& =\lambda_{q}+\left(m-\lambda_{p}\right)-m \\
& =\lambda_{q}-\lambda_{p} .
\end{aligned}
$$


The following corollary guarantees that there are no cycles in the flow lines of the gradient vector field.

Corollary 3.9. Given $f: M \rightarrow \mathbb{R}$ a Morse-Smale function, then the Morse index of the critical points of $f$ are strictly decreasing along the gradient flow, i.e., if $p$ and $q$ are critical points such that $W(q, p) \neq \varnothing$ then $\lambda_{p}<\lambda_{q}$.

Proof. Note that if $W(q, p) \neq \varnothing$ then $\operatorname{dim} W(q, p) \geq 1$, since $W(q, p)$ contains at least one gradient flow line which has dimension 1 . Then $\lambda_{q}-\lambda_{p} \geq 1$ and $\lambda_{p}<\lambda_{q}$.

Let us remark that given two manifolds $M$ and $N, M$ compact, there exists a metric $d_{1}$ on the space of $C^{1}$ maps, $C^{1}(M, N)$, that make it a complete metric space and further the topology generated by that metric has as subbasis the element of the $C^{1}$-Whitney topology. For more details the reader is invited to (HIRSCH, 2012) and for a short review (BANYAGA; HURTUBISE, 2013, Chapter 5, p. 140). Through this section we will consider the space $C^{1}(M, N)$ endowed with such metric but for the sake of simplicity we will omit details.

Definition 3.10. Let $N$ be a smooth manifold, $M$ and $Z$ be an embedded submanifolds with $M$ compact and the metric $d_{1}$ on $C^{1}(M, N)$. We said that $M$ is $\varepsilon C^{1}$-close to $Z$ if there exists a smooth map $h: M \rightarrow Z$ such that

$$
d_{1}\left(i_{M}, i_{Z} \circ h\right)<\varepsilon
$$

where $i_{M}$ and $i_{Z}$ are the inclusion maps.

Remark 3.11. Given $\varphi \in \operatorname{Diff}(M)$ it is known that:

1. the map $\bar{\varphi}: C^{1}(M, N) \rightarrow C^{1}(M, N), f \mapsto \varphi \circ f$, is a continuous map. Hence, for all $\varepsilon>0$ there exists $\delta>0$ such that if $M$ is $\delta C^{1}$-close to $Z$ then $\varphi(M)$ is $\varepsilon C^{1}$-close to $\varphi(Z)$;

2. denote by $\varphi^{n}=\underbrace{\varphi \circ \cdots \circ \varphi}_{n \text { times }}$ and $\varphi^{-n}=\underbrace{\varphi^{-1} \circ \cdots \circ \varphi^{-1}}_{n \text { times }}$ where $n \in \mathbb{N}$. Given $p \in M$ a hyperbolic fixed point, the stable manifold of $p$ is defined by

$$
W_{\varphi}^{s}(p)=\left\{x \in M \mid \lim _{n \rightarrow \infty} \varphi^{n}(x)=p\right\}
$$

and the unstable manifold of $p$ is defined by

$$
W_{\varphi}^{u}(p)=\left\{x \in M \mid \lim _{n \rightarrow-\infty} \varphi^{n}(x)=p\right\} .
$$

Lemma 3.12 ( $\lambda$-Lemma). Let $M$ be a smooth manifold and let $\varphi \in \operatorname{Diff}(M)$. Suppose that $p \in M$ is a hyperbolic fixed point of $\varphi$. If $N$ is an embedded submanifold of $M$ such that is invariant under $\varphi$ (i.e., $\varphi(N) \subset N$ ) and $q \in M$ is such that $N$ is transverse to $W_{\varphi}^{s}(p)$ at $q$, then for every $\varepsilon>0$ and for all $r$-cell $B \subset W_{\varphi}^{u}(p)$ there is an $r$-cell $D \subset N$ such that $B$ is $\varepsilon C^{1}$-close to $D$, where $r=\operatorname{dim} W_{\varphi}^{u}(p)$. 
Corollary 3.13. Let $M$ be a smooth manifold, suppose that $p$ and $q$ are hyperbolic fixed points of $\varphi \in \operatorname{Diff}(M)$. If there is a point $z \in W_{\varphi}^{u}(q) \cap W_{\varphi}^{S}(p)$ such that $W_{\varphi}^{u}(q)$ is transverse to $W_{\varphi}^{s}(p)$ at $z$, then $W_{\varphi}^{u}(p) \subset \overline{W_{\varphi}^{u}(q)}$.

Proof. Denote $r=\operatorname{dim} W_{\varphi}^{u}(p)$. Let $a \in W_{\varphi}^{u}(p)$, let $A, B \subset W_{\varphi}^{u}(p)$ be a $r$-cell neighborhood of $a$ and $p$ respectively. Since $W_{\varphi}^{u}(q)$ is transverse to $W_{\varphi}^{s}(p)$ at $z$ and $W_{\varphi}^{u}(q)$ is invariant under $\varphi$, from $\lambda$-Lemma we have that for any $\delta>0$ there exists a $r$-cell $D_{\delta} \subset W_{\varphi}^{u}(q)$ such that $B$ is $\delta C^{1}$-close to $D_{\delta}$. Since there is $n$ such that $\varphi^{-n}(A) \subset B, \varphi^{-n}(A)$ is $\delta C^{1}$-close to $D_{\delta}$. Hence, by Remark 3.11 for all $\varepsilon>0$ there exists $\delta>0$ such that $A$ is $\varepsilon C^{1}$-close to $\varphi^{n}\left(D_{\delta}\right) \subset W_{\varphi}^{u}(q)$. Therefore, there are point of $W_{\varphi}^{u}(q)$ arbitrarily close to $a \in A$.

Using the Theorem 1.48 and the $\lambda$-Lemma it can be proved that.

Proposition 3.14. Let $M$ be a smooth manifold and $p_{1}, p_{2}, p_{3} \in M$ be hyperbolic fixed points of $\varphi \in \operatorname{Diff}(M)$. Suppose that $W_{\varphi}^{u}\left(p_{3}\right) \pitchfork W_{\varphi}^{s}\left(p_{2}\right)$ and $W_{\varphi}^{u}\left(p_{2}\right) \pitchfork W_{\varphi}^{s}\left(p_{1}\right)$ and denote by $W_{\varphi}\left(p_{i}, p_{j}\right)=$ $W_{\varphi}^{u}\left(p_{i}\right) \cap W_{\varphi}^{s}\left(p_{j}\right)$ for $i \neq j$. Then

$$
\left\{p_{1}, p_{2}, p_{3}\right\} \cup W_{\varphi}\left(p_{2}, p_{1}\right) \cup W_{\varphi}\left(p_{3}, p_{2}\right) \subset \overline{W_{\varphi}\left(p_{3}, p_{1}\right)} .
$$

In the particular case where $f: M \rightarrow \mathbb{R}$ is a Morse-Smale function by Proposition 2.12 the critical points of $f$ are hyperbolic fixed point of the gradient flow $\phi_{t}$ for each $t \in \mathbb{R}$ and hence we can consider $W_{\phi_{0}}^{u}(p)=W^{u}(p)$. Thus the proposition above can be formulated as follows.

Corollary 3.15. Let $f: M \rightarrow \mathbb{R}$ be a Morse-Smale function on a $m$-dimensional compact smooth Riemannian manifold. Let $p, q, r \in M$ be critical points, if $W(r, q) \neq \varnothing$ and $W(q, p) \neq \varnothing$ then $W(r, p) \neq \varnothing$ and

$$
\{p, q, r\} \cup W(r, q) \cup W(q, p) \subseteq \overline{W(r, p)} .
$$

Definition 3.16. Let $f: M \rightarrow \mathbb{R}$ be a Morse-Smale function and $p, q \in M$ be critical points of $f$. We say that $q$ is succeeded by $p, q \geq p$, if $W(q, p) \neq \varnothing$.

Note that $\geq$ is a partial order in $\operatorname{Cr}(f)$, the set of critical points of $f$. The pair $(C r(f), \geq)$ is called phase diagram of $f$. A consequence of the previous results is the following.

Proposition 3.17. Let $f: M \rightarrow \mathbb{R}$ be a Morse-Smale function and $p \in M$ be critical point. Then

$$
\begin{gathered}
\overline{W^{u}(q)}=\bigcup_{q \geq r} W^{u}(r), \\
\overline{W(q, p)}=\bigcup_{q \geq q^{\prime} \geq p^{\prime} \geq p} W\left(q^{\prime}, p^{\prime}\right) .
\end{gathered}
$$

Now we can prove the main theorem in this section which states that the number of flow lines of a Morse-Smale function between points with consecutive Morse index is finite. 
Theorem 3.18. Suppose that $\lambda_{q}-\lambda_{p}=1$ and $W(q, p) \neq \varnothing$, then

$$
\overline{W(q, p)}=W(q, p) \cup\{p, q\},
$$

and $W(q, p)$ has a finite number of components.

Proof. Since $\lambda_{q}-\lambda_{p}=1$, there are not critical points other than $q$ and $p$ between $q$ and $p$, thus by Proposition 3.17

$$
\overline{W(q, p)}=W(q, p) \cup W(q, q) \cup W(p, p)=W(q, p) \cup\{p, q\} .
$$

For $x \in W(q, p)$ denote $\gamma_{x}$ the gradient flow through $x$. Note that, if $y \in \gamma_{x}$ then $\gamma_{x}=\gamma_{y}$. Then $\cup_{x \in W(q, p)} \gamma_{x}$ is an open cover of $W(q, p)$. Let $U$ and $V$ be a small open set of $p$ and $q$ respectively. Then

$$
\bigcup_{x \in W(q, p)} \gamma_{x} \cup U \cup V
$$

is an open cover of $\overline{W(q, p)}$. Since $\overline{W(q, p)}$ is closed and $M$ is compact, $\overline{W(q, p)}$ is compact. Therefore, it has a finite subcover $\gamma_{1} \cup \cdots \cup \gamma_{k} \cup U \cup V$, thus $\gamma_{1} \cup \cdots \cup \gamma_{k}$ is a cover of $W(q, p)$. It means that $W(q, p)$ has finite components and thus there will be a finite number of gradient line flow between $q$ and $p$.

Remark 3.19. It is important to point out that given a Morse-Smale function $f: M \rightarrow \mathbb{R}$ by Proposition 3.2 we have

$$
M=\bigsqcup_{p \in C r(f)} W^{u}(p) .
$$

This decomposition of $M$ by the unstable manifolds indicates a strong relationship with the CW-complex structure on $M$ as in Theorem 2.21. In fact, the called Cell Equivalence Theorem (FRANKS, 1979) (see also (BANYAGA; HURTUBISE, 2013, Theorem 6.34, p. 178 )) shows that there is a CW-complex $X$ such that this relation is not a mere coincidence. Indeed, (as in Theorem 2.18) the $\lambda$-cell is given by the unstable manifold $W^{u}(p)$ as follows

$$
e^{\lambda}=W^{u}(p) \cap \overline{M \backslash M^{c-\varepsilon}},
$$

and the attaching map given by the inclusion $\partial e^{\lambda} \hookrightarrow \partial M^{c-\varepsilon}$, where $c=f(p)$. This provides a $\mathrm{CW}$-complex structure $X$, unique up to cell equivalence and a homotopy equivalence $h: M \rightarrow X$, such that for a each critical point $p$ of index $\lambda$ we have $h\left(W^{u}(p)\right)=X\left(e^{\lambda}\right)$, where $X\left(e^{\lambda}\right)$ is the smallest ${ }^{1}$ subcomplex of $X$ containing $e^{\lambda}$.

Moreover, the converse problem is also interesting, i.e., whether a CW-complex homotopy equivalent to $M$ is the complex associated to some Morse-Smale flow on $M$. This problem was answer by Franks (FRANKS, 1979, Theorem 4.4. p. 208) for manifolds with dimension grater than five.

\footnotetext{
$\overline{1}$ for a convenient partial ordering on the cells of $X$.
} 


\subsection{Morse Homology Theorem}

We have already seen all the necessary tools to define a chain complex called the MorseSmale-Witten chain complex. But first we need some conventions for the orientation of the stable and unstable manifolds and its intersections of a given Morse-Smale function $f: M \rightarrow \mathbb{R}$ where $M$ is an oriented compact Riemmanian $m$-manifold.

- We fix an orientation on $W^{u}(r)$ for each critical point $r$ of $M$. Since $W^{u}(r) \pitchfork W^{s}(r)$, an orientation on $W^{u}(r)$ induces an orientation on $W^{s}(r)$.

- The manifold $W(q, p)$ has an orientation induced by the oriented intersection of $W^{u}(q)$ and $W^{s}(p)$. Namely, for $x \in W(q, p)$ let $N_{x}\left(W(q, p), W^{u}(q)\right)$ be the orthonormal complement of $T_{x}(W(q, p))$ in $W^{u}(q)$, then

$$
N_{x}\left(W(q, p), W^{u}(q)\right) \oplus T_{x} W^{s}(p)=T_{x} M,
$$

So, $N_{x}\left(W(q, p), W^{u}(q)\right)$ has an orientation induced by orientations on $M$ and $W^{s}(p)$. We have that

$$
N_{x}\left(W(q, p), W^{u}(q)\right) \oplus T_{x} W(q, p)=T_{x} W^{u}(q) .
$$

Then $T_{x} W(q, p)$ has an orientation induced by orientations on $W^{u}(q)$ and $N_{x}\left(W(q, p), W^{u}(q)\right)$.

- For a regular value $c \in \mathbb{R}$ between $f(p)$ and $f(q)$ we have an orientation on the level set $f^{-1}(c)$ induced by $-\nabla f$ (see Example 1.46), i.e., the base $\left(v_{1}, \ldots, v_{m-1}\right)$ is a positive orientation of $T_{x} f^{-1}(c)$ if the base $\left(-\nabla f_{x}, v_{1}, \ldots, v_{m}\right)$ is a positive orientation $T_{x} M$.

Let $G_{k}(f)$ be the free abelian group generated by the set of critical points of index $k, C r_{k}(f)$, then

$$
G_{k}(f)=\left\{\sum_{p \in C r_{k}(f)} a_{p} p \mid a_{p} \in \mathbb{Z}\right\}
$$

Define the boundary operator,

$$
\begin{aligned}
\partial: G_{k}(f) & \rightarrow G_{k-1}(f) \\
a & \mapsto \sum_{b \in C r_{k-1}(f)} n(a, b) b
\end{aligned}
$$

where

$$
n(a, b)=\sum_{x \in f^{-1}(c) \cap W(a, b)} \operatorname{sign}(x) .
$$

Note that the points of the oriented intersection $f^{-1}(c) \cap W(a, b)$ is in one-to-one correspondence with set of flow lines that go from $a$ to $b$. Then, by Theorem 3.18 the set $f^{-1}(c) \cap W(a, b)$ is finite and hence the sum in (3.2) is finite and therefore the number $n(a, b)$ is well defined. Also note that $n(a, b)$ is the oriented intersection number of $W(a, b)$ and $f^{-1}(c)$ as introduced in Definition 1.50.

Now we will state the main theorem of this chapter. 
Theorem 3.20 (Morse homology theorem). $\left(G_{*}(f), \partial\right)$ is a chain complex, i.e., $\partial \circ \partial=0$. Its homology is isomorphic to the singular homology $H_{\star}(M, \mathbb{Z})$.

The chain complex $\left(G_{\star}(f), \partial\right)$ is called Morse-Smale-Witten chain complex and its homology is called the Morse homology. 

ANDRICA, D.; ALDEA, M. Perfect Morse functions and some applications. Acta Univ. Apulensis Math. Inform., n. 8, p. 16-25, 2004. Citation on page 60.

ANICK, D. J. Recent progress in Hilbert and Poincaré series. In: Algebraic topology—rational homotopy (Louvain-la-Neuve, 1986). Springer, Berlin, 1988, (Lecture Notes in Math., v. 1318). p. 1-25. Available: <https://doi.org/10.1007/BFb0077791>. Citation on page 56.

BANYAGA, A.; HURTUBISE, D. Lectures on Morse Homology. [S.1.]: Springer Netherlands, 2013. (Texts in the Mathematical Sciences). ISBN 9781402026966. Citations on pages 34, 35, $43,63,66,67$, and 69.

BELLMAN, R. Introduction to Matrix Analysis (2nd Ed.). USA: Society for Industrial and Applied Mathematics, 1997. ISBN 0898713994. Citations on pages 24 and 25.

BREDON, G. E. Topology and geometry. New York: Springer-Verlag, 1993. (Graduate texts in mathematics ; 139). ISBN 3540979263. Citation on page 13.

FRANKS, J. M. Morse-smale flows and homotopy theory. Topology, v. 18, n. 3, p. 199-215, 1979. ISSN 0040-9383. Citation on page 69.

GREENBERG, M. J. Lectures on algebraic topology. [S.1.]: W. A. Benjamin New York, 1967. x, 235 p. p. Citations on pages 13 and 25 .

GUILLEMIN, V. W.; POLLACK, A. Differential topology. [S.1.]: Prentice-Hall Englewood Cliffs, N.J, 1974. xvi, 222 p. p. ISBN 0132126052. Citations on pages 13 and 36.

HATCHER, A. Algebraic Topology. [S.1.]: Cambridge University Press, 2002. (Algebraic Topology). ISBN 9780521795401. Citations on pages 13, 25, 31, and 33.

HIRSCH, M. Differential Topology. [S.1.]: Springer New York, 2012. (Graduate Texts in Mathematics). ISBN 9781468494495. Citation on page 67.

LEE, J. Introduction to Smooth Manifolds. [S.1.]: Springer New York, 2013. (Graduate Texts in Mathematics). ISBN 9780387217529. Citations on pages 13, 15, 16, 18, 34, and 35.

MILNOR, J. Differential topology. [S.1.: s.n.], 1964. 165-185 p. Citation on page 51.

Morse Theory. [S.1.]: Princeton University Press, 2016. (Annals of Mathematics Studies, v. 51). ISBN 9781400881802. Citations on pages 11 and 13.

MORSE, M. The foundations of the calculus of variations in the large in m-space (first paper). Transactions of the American Mathematical Society, v. 31, p. 379-404, 1929. Citation on page 11 .

SMALE, S. Differentiable dynamical system. Bull. Am. Math. Soc, v. 73, p. 747-817, 1967. Citation on page 11. 
THOM, R. Sur une partition en cellules associee à une fonction sur une variété. C. R. Acad. Sci., Paris, Gauthier-Villars, Paris, v. 228, p. 973-975, 1949. ISSN 0001-4036. Citation on page 11. 


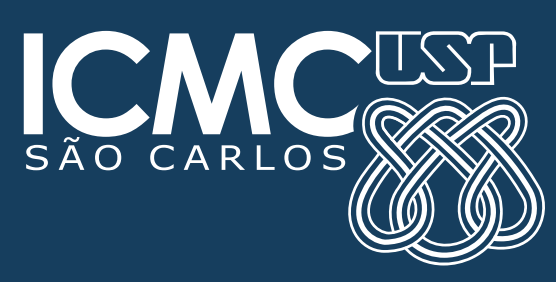

